MATHEMATICS OF COMPUTATION

Volume 70, Number 235, Pages 977-1029

S 0025-5718(00)01218-7

Article electronically published on February 17, 2000

\title{
A CONVERGENT BOUNDARY INTEGRAL METHOD FOR THREE-DIMENSIONAL WATER WAVES
}

\author{
J. THOMAS BEALE
}

\begin{abstract}
We design a boundary integral method for time-dependent, threedimensional, doubly periodic water waves and prove that it converges with $O\left(h^{3}\right)$ accuracy, without restriction on amplitude. The moving surface is represented by grid points which are transported according to a computed velocity. An integral equation arising from potential theory is solved for the normal velocity. A new method is developed for the integration of singular integrals, in which the Green's function is regularized and an efficient local correction to the trapezoidal rule is computed. The sums replacing the singular integrals are treated as discrete versions of pseudodifferential operators and are shown to have mapping properties like the exact operators. The scheme is designed so that the error is governed by evolution equations which mimic the structure of the original problem, and in this way stability can be assured. The wavelike character of the exact equations of motion depends on the positivity of the operator which assigns to a function on the surface the normal derivative of its harmonic extension; similarly, the stability of the scheme depends on maintaining this property for the discrete operator. With $n$ grid points, the scheme can be implemented with essentially $O(n)$ operations per time step.
\end{abstract}

\section{INTRODUCTION}

In this paper we design a semidiscrete numerical method of boundary integral type for computing time-dependent, doubly periodic, three-dimensional water waves. We prove that this method converges as long as the actual motion remains smooth, without restriction on its amplitude. While the full equations are difficult to work with analytically, boundary integral methods seem naturally suited to water wave motion. The surface is represented in Lagrangian coordinates, and grid points are moved according to a computed velocity. Because the flow is assumed irrotational, the motion is determined by quantities on the surface alone. However, the normal velocity must be found in terms of singular boundary integrals, using potential theory. The evolution has the character of nonlinear, nonlocal wave motion without dissipation. For this reason it seems that the stability of the numerical method depends critically on its design. In the present work we develop a new, efficient approach for calculating the singular integrals, using the limited information computed on the moving surface. We analyze discrete versions of integral operators such as single and double layer potentials by adapting the viewpoint

Received by the editor September 9, 1998 and, in revised form, June 10, 1999.

2000 Mathematics Subject Classification. Primary 65M12, 76B15; Secondary 65D30.

Key words and phrases. Water waves, boundary integral methods, integral operators, quadrature of singular integrals.

The author was supported in part by NSF Grant \#DMS-9870091. 
of pseudodifferential operators, as well as potential theory, to the discrete setting. We find the most important terms in the evolution equations for the errors, and ensure by design of the scheme that the errors grow at a bounded rate.

Boundary integral methods have been widely used in ocean engineering and applied mathematics, starting with the work of Longuet-Higgins and Cokelet [21] and Vinje and Brevig 33. Two-dimensional applications have been extensive; see the recent surveys [27, 32] or the references in [5, 6]. Computations in 3-D include 4, 27, 28, $9,32,17$. In [6, T. Hou, J. Lowengrub, and the author proved the convergence of a version of the method in 2-D. Calculations illustrate the advantage of this method in capturing small scale features. It seems that convergence in $3-\mathrm{D}$ is more difficult, largely because quadrature for singular integrals on surfaces is more involved than on curves, and because the choice of quadrature affects the stability of the time-dependent solution. One approach is presented in [19].

We assume as usual that the flow of the water is incompressible, inviscid, and irrotational. For simplicity we assume the fluid is infinitely deep and the motion is periodic in both horizontal directions, with period $2 \pi$. (The formulation can easily be modified to account for a horizontal bottom; for more general lower boundaries, see [3.) The motion of the fluid is governed by Euler's equations and boundary conditions at the surface. Since the flow is irrotational and incompressible, the velocity is the gradient of a potential $\phi$ which is harmonic below the surface:

$$
\Delta \phi=0 \text {. }
$$

At the surface the fluid pressure matches the atmospheric pressure, which we assume constant. (We neglect surface tension.) Bernoulli's equation, together with the boundary condition for the pressure, provides us with an evolution equation for $\phi$ at the surface. With the position $x$ and velocity potential $\phi$ at the surface treated as functions of Lagrangian coordinates $\alpha=\left(\alpha_{1}, \alpha_{2}\right)$ and time $t$, the evolution equations are

$$
x_{t}=v, \quad \phi_{t}=\frac{1}{2}|v|^{2}-g x_{3},
$$

where $v$ is the fluid velocity, $g$ is the acceleration of gravity, $x_{3}$ is the vertical component of $x$, and $\phi$ is adjusted by a spatial constant at each $t$. The first equation says that a particle on the surface with fixed $\alpha$ moves with the fluid velocity. The velocity $v=\nabla \phi$ is determined by $\phi$ on the surface and the condition (1.1), with the requirement that $\nabla \phi$ is square integrable as $x_{3} \rightarrow-\infty$.

In the boundary integral approach, the main effort in solving these equations is in finding the velocity $v$ at the surface using potential theory. There is some choice in how this is done. The tangential part of the velocity can be found from differentiating $\phi$ along the surface. For the normal component we use a Fredholm integral equation of the second kind which determines the normal velocity $\phi_{n}=$ $\partial \phi / \partial n$ directly from $\phi$ on the surface,

$$
\begin{aligned}
& \frac{1}{2} \phi_{n}(x)+\int \frac{\partial G^{\pi}(x-y)}{\partial n(x)} \phi_{n}(y) d S(y) \\
&=n(x) \cdot \int \nabla G^{\pi}(x-y) \times\left(\left(n(y) \times \nabla^{T} \phi(y)\right) d S(y) .\right.
\end{aligned}
$$

Here $G^{\pi}$ is the Green's function, periodic in the horizontal variables, obtained from a sum of images of the standard Green's function $G(x)=-1 / 4 \pi|x|$ with $\Delta G=\delta ; n$ is the unit normal pointing out of the fluid. We assume $\phi$ is periodic and has square 
integrable gradient below the surface. The first integral is absolutely convergent, but the second is of principal type. The right side is the normal derivative of the double layer potential due to $\phi$. This integral equation has been used in electromagnetics. It can be derived by writing $\phi$ below the surface in terms of boundary integrals in $\phi$ and $\phi_{n}$, and then taking the normal derivative at the surface (e.g., see [10], $§ 3.9$ and Theorem 2.23, or [20]). Its use for three-dimensional water waves has been suggested in [28. An alternative approach is to write the velocity potential in terms of a dipole source on the boundary; this was done in $3,4,4,17,5,6$.

To design a specific scheme, we have choices to make in writing the integrals, finding derivatives, and especially in the numerical integration of the singular integrals. Analytical considerations in the present work and in [6 indicate that these choices can determine whether or not the scheme is stable. There are well developed methods for quadrature of singular integrals in several dimensions which depend on special coordinates near the singularity; see Lyness [24 for a survey. In the present context the surface and integrand are known only from computed values at the Lagrangian grid points. Here we develop a general approach in which we replace the Green's function $G$ by a smooth version $G_{h}$ regularized on the scale of the grid size $h$. We discretize the new integral by the trapezoidal rule. Then, using an asymptotic expansion of the quadrature error like that found by Lyness [23] and Goodman et al. [12] for unregularized singular integrals, we identify the largest error term. Because of the regularization, we can compute this first error and correct for it; it is expressed through the Poisson summation formula in terms of the Fourier transform $\hat{G}_{h}$ of $G_{h}$. In our case the correction improves an $O(h)$ error to $O\left(h^{3}\right)$. (We have avoided using extrapolation as in 23] for the sake of a positivity requirement explained below.)

As in 6] we are guided in designing the scheme by the linearization of the exact equations about an arbitrary solution. By preserving the same structure at the discrete level we can ensure that the numerical scheme is stable. Without such considerations we could have a mismatch of terms which destroys the well-posedness of the linearization and therefore the numerical stability. Briefly, this linearization reduces to an equation for a variable $u$ related to the disturbance in the velocity potential on the surface,

$$
u_{t t}+c \Lambda u \approx 0
$$

where we have dropped less important terms, and $\Lambda$ is the principal part of the Dirichlet-to-Neumann operator on the current surface, i.e., the operator that determines $\phi_{n}$ from $\phi$. It is important that $\Lambda$ and $c$ are positive, as discussed further below. The equation describes nonlocal wave motion; in the special case of small disturbances at equilibrium, it reduces to the usual equation for linearized water waves. The linearization about an arbitrary motion was found in the boundary integral setting in 2-D in [5], and in 3-D with orthogonal coordinates in [18]. It was derived earlier for bounded domains in a different way in [2].

A primary concern is that the discrete version of the operator $\Lambda$ should be positive, or almost so. Violation of this requirement in high wavenumbers would lead to rapid growth of disturbances, as can be seen from (1.4). For the exact operator $\Lambda$, the positivity is related to the fact that the Green's function has transform $\hat{G}<0$. This property may not be preserved by the discrete analogue. In the present approach, we choose $G_{h}$ so that $\hat{G}_{h}<0$. The discrete transform is a lattice sum of 
values of $\hat{G}_{h}$, which converges because $G_{h}$ is regularized. In this way we can ensure the necessary positivity.

We now describe the method in more detail. We first rewrite the integrals in (1.3) to reduce the order of singularity, taking advantage of the two identities

$$
\int \frac{\partial G^{\pi}(x-y)}{\partial n(y)} d S(y)=0, \quad \int \nabla G^{\pi}(x-y) \times n(y) d S(y)=0,
$$

where the integral is over one period of the surface. (Cf. [4], $\S 3$ or [10], Theorem 2.1. The use of these identities to reduce the singularity was suggested in 44.) On the right side of (1.3) we use vector identities to convert the integrand,

$$
\begin{aligned}
\nabla G^{\pi} \times\left(n \times \nabla^{T} \phi\right)=\left(\nabla G^{\pi} \cdot \nabla^{T} \phi\right) & n-\left(\nabla G^{\pi} \cdot n\right) \nabla^{T} \phi \\
& =\left(\nabla G^{\pi} \times n\right) \times \nabla^{T} \phi-\left(\nabla G^{\pi} \cdot n\right) \nabla^{T} \phi .
\end{aligned}
$$

Then using (1.5) we can write the integral on the right in (1.3) as

$$
\begin{aligned}
\int\left(\nabla G^{\pi} \times n(y)\right) \times\left(\nabla^{T} \phi(y)\right. & \left.-\nabla^{T} \phi(x)\right) d S(y) \\
& -\int \nabla G^{\pi} \cdot n(y)\left(\nabla^{T} \phi(y)-\nabla^{T} \phi(x)\right) d S(y) .
\end{aligned}
$$

The first integral is absolutely convergent because of the subtraction. We can replace $\nabla G^{\pi}$ by $\nabla^{T} G^{\pi}$, the part of the gradient tangential at $y$, and write (cf. [10], p. 35), with $y=y\left(\alpha^{\prime}\right)$ and $X_{1}, X_{2}$ the tangent vectors $X_{j}=\partial y / \partial \alpha_{j}^{\prime}$,

$$
\nabla G^{\pi}(x-y) \times N(y)=\left(D_{\alpha_{1}^{\prime}} G^{\pi}\right) X_{2}-\left(D_{\alpha_{2}^{\prime}} G^{\pi}\right) X_{1} .
$$

Here $N=X_{1} \times X_{2}$; we assume $N$ points outward, so that $n=N /|N|$. For the integral on the left in (1.3), we resolve $\nabla G^{\pi}$ into parts normal and tangential at $y$ and use 1.5) again, so that the integral becomes

$$
\int \nabla G^{\pi} \cdot n(y)\left[n(y) \cdot n(x) \phi_{n}(y)-\phi_{n}(x)\right] d S(y)+\int \nabla^{T} G^{\pi} \cdot n(x) \phi_{n}(y) d S(y) .
$$

The second integrand is absolutely integrable, since $\nabla^{T} G^{\pi} \perp n(x)$ at $y=x$.

We work with grid functions on a grid of size $h$ representing equally spaced values of the Lagrangian variable, $\alpha_{j}=j h$, where $j=\left(j_{1}, j_{2}\right) \in \mathbf{Z}^{2}$. We assume $h=2 \pi / N$ with $N$ even. For the discrete values in a fundamental period we take

$$
I=\left\{j \in \mathbf{Z}^{2}:-N / 2 \leq j_{\nu} \leq N / 2-1, \nu=1,2\right\} .
$$

A grid function $f$ has a discrete Fourier transform which we denote by $\ddot{f}$, a function of $k \in \mathbf{Z}^{2}$ with period $2 \pi / h$ in $k_{1}, k_{2}$,

$$
\ddot{f}(k)=(2 \pi)^{-2} \sum_{j \in I} f(j h) e^{-i k j h} h^{2}, \quad f(j h)=\sum_{k \in I} \ddot{f}(k) e^{i k j h} .
$$

For properties of this transform see e.g., [15, 31]. We measure grid functions on $I h$ in the discrete $L_{h}^{2}$ norm, given by

$$
|f|_{L_{h}^{2}}^{2}=\sum_{j \in I}|f(j h)|^{2} h^{2}=(2 \pi)^{2} \sum_{k \in I}|\ddot{f}(k)|^{2} .
$$

Of the exact solution, we assume that the surface function $x(\alpha, t)$ and the velocity potential $\phi(\alpha, t)$ are smooth up to some time $T$, and $x\left(\alpha_{1}, \alpha_{2}, t\right)-\left(\alpha_{1}, \alpha_{2}, 0\right)$ and $\phi\left(\alpha_{1}, \alpha_{2}, t\right)$ are $2 \pi$-periodic in each of $\alpha_{1}, \alpha_{2}$. (If the velocity is periodic but $\phi$ is 
not, we can choose a frame of reference so that $\phi$ becomes periodic. The scheme can easily be adapted to the more general case.) We also assume that the Jacobian $\partial x / \partial \alpha$ is nondegenerate and $x(\alpha, t) \neq x\left(\alpha^{\prime}, t\right)$ when $\alpha \neq \alpha^{\prime}$. This implies that

$$
\left|x(\alpha, t)-x\left(\alpha^{\prime}, t\right)\right| \geq c\left|\alpha-\alpha^{\prime}\right|, \quad 0 \leq t \leq T .
$$

Next we discuss the discrete derivative operator. It can be a difference operator, but to be general we discuss its Fourier representation. For a function $f$ of $\alpha_{j} \in I h$, we assume the discrete partial derivative $D_{h, \nu}$ in $\alpha_{\nu}, \nu=1,2$, has the form

$$
\left(D_{h, \nu} f\right)^{\cdot *}(k)=h^{-1} \sigma\left(k_{\nu} h\right) \ddot{f}(k), \quad \sigma(\xi+2 \pi)=\sigma(\xi),
$$

where $\sigma: \mathbf{R} \rightarrow \mathbf{C}$. We also assume

$$
\begin{gathered}
\sigma \text { is } C^{1} \text { on }[-\pi, \pi], \quad \sigma(-\pi)=\sigma(\pi), \quad C_{1}|\xi| \leq|\sigma(\xi)| \leq C_{2}|\xi| \text { on }[-\pi, \pi] \\
\sigma(\xi)=i \xi+O\left(\xi^{4}\right) \text { as } \xi \rightarrow 0, \quad \sigma(-\xi)=\bar{\sigma}(\xi)
\end{gathered}
$$

where $\bar{\sigma}$ is the complex conjugate. The latter says that $D_{h, \nu}$ is accurate to $O\left(h^{3}\right)$ and preserves real-valued functions. Because of (1.15), $D_{h, \nu}$ has a product rule: for $f \in L_{h}^{2}$ and $u$ a smooth periodic function,

$$
D_{h, \nu}(u f)=u D_{h, \nu} f+B(f),
$$

where $B$ is an operator depending on $u$, bounded on $L_{h}^{2}$ uniformly in $h$ (see [6], Lemma 1). This will be useful later. The requirement $\sigma(\xi) \neq 0$ for $\xi \neq 0$ rules out, e.g., the symmetric fourth order difference operator. A simple example meeting these conditions is the third order difference operator coming from Lagrange interpolation,

$$
D_{h, \nu}=(6 h)^{-1}\left(-2 S_{\nu}^{-1}-3 I+6 S_{\nu}-S_{\nu}^{2}\right)
$$

where $S_{\nu}$ is the shift operator, $\left.S_{1} f\left(j_{1} h, j_{2} h\right)=f\left(\left(j_{1}+1\right) h, j_{2} h\right)\right)$. Alternatively, we could define $\sigma$ directly, e.g., by setting $\sigma=i \xi \rho+(1-\rho)$ for $|\xi| \leq \pi$, where $\rho(\xi)$ is some even, real-valued function with $\rho \equiv 1$ near $\xi=0$ and $\rho \equiv 0$ near $\xi= \pm \pi$. We shall refer to the adjoint operator $D_{h, \nu}^{*}$, given by

$$
\left(D_{h, \nu}^{*} f\right)^{*}(k)=h^{-1} \bar{\sigma}\left(k_{\nu} h\right) \ddot{f}(k) .
$$

Of course, for (1.18), $D_{h, \nu}^{*}$ is found by replacing $S_{\nu}$ with $S_{\nu}^{-1}$. For $f$ smooth, $D_{h, \nu} f=\partial f / \partial \alpha_{\nu}+O\left(h^{3}\right)$ while $D_{h, \nu}^{*}=-\partial f / \partial \alpha_{\nu}+O\left(h^{3}\right)$.

Various geometric quantities must be computed from the grid points on the surface. From $x\left(\alpha_{j}\right), j \in I$, we can use $D_{h, \nu}$ to compute approximate tangent vectors $X_{\nu j}=\left(D_{h, \nu} x\right)_{j}, \nu=1,2$. (We apply $D_{h, \nu}$ to $x(\alpha)-(\alpha, 0)$.) From these we compute the metric tensor $g_{\mu \nu}=X_{\mu} \cdot X_{\nu}$ and the inverse $g^{\mu \nu}$, as functions of $\alpha_{j}$. We then find the normal vectors $N=X_{1} \times X_{2}$ and $n=N /|N|$, and also the dual tangent vectors at $\alpha_{j}$,

$$
X_{\mu}^{*}=\sum_{\nu} g^{\mu \nu} X_{\nu}, \quad X_{\mu}^{*} \cdot X_{\nu}=\delta_{\mu \nu}
$$

Given the surface $x$ and the velocity potential $\phi$ at the surface at one time for the grid points $\alpha_{j}, j \in I$, we can advance to the next time using (1.2) once we have computed the velocity $v$ at each $\alpha_{j}$. To do this we write the computed velocity as

$$
v=\nabla_{h}^{T} \phi+w n,
$$


where $w$ is an approximation to $\phi_{n}$. We can compute the tangential gradient as (e.g., cf. [10], p. 33)

$$
\nabla_{h}^{T} \phi=\sum_{\nu=1,2}\left(D_{h, \nu} \phi\right) X_{\nu}^{*}
$$

We will obtain $w$ from a discrete version of (1.3), rewriting the two integrals as in (1.7) - (1.9). We use the regularized Green's function $G_{h}^{\pi}$; for the normal derivatives we use the analytic gradient of $G_{h}^{\pi}$, while for $\nabla^{T} G_{h}^{\pi}\left(x-x\left(\alpha^{\prime}\right)\right)$, the part of the gradient tangential at $x\left(\alpha^{\prime}\right)$, we use the discrete $\alpha^{\prime}$-derivative,

$$
\nabla_{h}^{T} G_{h}^{\pi}\left(x-x\left(\alpha^{\prime}\right)\right)=-\sum_{\nu=1,2}\left(D_{h, \nu} G_{h}^{\pi}\left(x-x\left(\alpha^{\prime}\right)\right)\right) X_{\nu}^{*} .
$$

This expression is used to match (1.22); both appear in the discrete operator $\Lambda$ mentioned above, and this symmetry gives it the proper structure.

The regularized Green's function $G_{h}^{\pi}$ is constructed in $\$ 2$ from a free space version in the form $G_{h}(x)=-(4 \pi|x|)^{-1} s(|x| / h)$ for $x \in R^{3}$, where $s$ is a function chosen with certain conditions (2.2), (2.3), (2.9) which ensure that $G_{h}$ is smooth and differs from $G$ itself as an integral operator by $O\left(h^{3}\right)$. As above, we also assume that $\hat{G}_{h}(k)<0$. A specific choice (2.19) for $s$ is described in 92 , based on the Gaussian function, for which the requirements are satisfied and necessary quantities can be computed explicitly. Since the regularization has length scale $h, G_{h}$ differs appreciably from $G$ only within distance $O(h)$ of the origin. If, for example, $x(\alpha)=$ $\left(\alpha_{1}, \alpha_{2}, 0\right)$ and we approximate the single layer potential due to a function $f$ by applying the trapezoidal rule with $G_{h}$ in place of $G$, we have (with a limiting value at $\ell=j$ )

$$
\sum_{\ell \in \mathbf{Z}^{2}} G((j h-\ell h, 0)) s(j-\ell) f(\ell h) h^{2},
$$

and the $s$ factor amounts to a set of quadrature weights. The quadrature error is $O(h)$; however, it can be improved to $O\left(h^{3}\right)$ by a correction proportional to $f(j h)$, as explained in $\$ 3$. Such corrections appear in the discrete integrals below.

The discrete integral equation is obtained by substituting (1.7), (1.9) into (1.3) for the two integrals, replacing $G^{\pi}$ with $G_{h}^{\pi}$, applying the trapezoidal rule, and adding the needed quadrature correction. It has the form

$$
\begin{aligned}
& \left(\mathcal{K}_{h} w\right)_{j}=\sum_{\ell} \nabla G_{h}^{\pi} \cdot N_{\ell}\left[\left(n_{\ell} \cdot n_{j}\right) w_{\ell}-w_{j}\right] h^{2}+\sum_{\ell} \nabla_{h}^{T} G_{h}^{\pi} \cdot n_{j} w_{\ell}\left|N_{\ell}\right| h^{2}+h \kappa_{j} w_{j} \\
& (1.27) \\
& F_{j}=\sum_{\ell}\left[D_{h} G_{h}^{\pi}, X\right]_{\ell} \times\left(\nabla_{h}^{T} \phi_{\ell}-\nabla_{h}^{T} \phi_{j}\right) h^{2}+h \chi_{j}-\sum_{\ell} \nabla G_{h}^{\pi} \cdot N_{\ell}\left(\nabla_{h}^{T} \phi_{\ell}-\nabla_{h}^{T} \phi_{j}\right) h^{2}
\end{aligned}
$$

Here $\nabla_{h}^{T} \phi$ is found from (1.22) and $\nabla_{h}^{T} G_{h}^{\pi}$ from (1.23); $\nabla G_{h}^{\pi}$ etc. are evaluated at $x_{j}-x_{\ell}$; and in view of (1.8) we have introduced

$$
\left[D_{h} G_{h}^{\pi}, X\right]_{\ell}=D_{h, 1} G_{h}^{\pi}\left(x_{j}-x_{\ell}\right) X_{2 \ell}-D_{h, 2} G_{h}^{\pi}\left(x_{j}-x_{\ell}\right) X_{1 \ell},
$$


where $D_{h}$ is with respect to $\ell$. The correction terms are given by

$$
\begin{gathered}
\kappa_{j}=\gamma_{j}\left[P_{h}\left(D_{h, 1}^{*} X_{1}^{*}+D_{h, 2}^{*} X_{2}^{*}\right)\right]_{j} \cdot N_{j}, \\
\chi_{j}=\gamma_{j}\left(X_{1 j} \times\left[P_{h} D_{h, 2}^{*}\left(\nabla_{h}^{T} \phi\right)\right]_{j}-X_{2 j} \times\left[P_{h} D_{h, 1}^{*}\left(\nabla_{h}^{T} \phi\right)\right]_{j}\right),
\end{gathered}
$$

where

$$
\gamma_{j}=2 \pi\left(\operatorname{det} g^{\mu \nu}\left(\alpha_{j}\right)\right)^{1 / 2} \sum_{0 \neq n \in \mathbf{Z}^{2}} \Gamma\left(2 \pi \sqrt{g^{\mu \nu}\left(\alpha_{j}\right) n_{\mu} n_{\nu}}\right)
$$

and $\Gamma$ is a function related to $G_{h}$; see (2.14), (2.24). The sum in (1.31) is infinite, but we can choose $G_{h}$ so that $\Gamma$ decreases rapidly and only a few terms are needed (cf. (2.24)). The derivation of these corrections is explained in $\$ 3$. A linear operator $P_{h}$ is inserted in (1.29), (1.30) to smooth out the second derivatives. We assume it has the form

$$
\left(P_{h} f\right)^{\cdot *}(k)=\rho(k \sqrt{h}) \ddot{f}(k)
$$

with $\rho$ chosen so that

$$
\rho(\xi)=1+O\left(|\xi|^{4}\right) \text { as } \xi \rightarrow 0, \quad \rho(\xi)=0 \text { for }|\xi|>c_{0}, \quad c_{0}>0 .
$$

As part of the convergence argument, we prove that the integral equation can be solved by simple iteration, i.e.,

$$
\frac{1}{2} w^{(n+1)}+\mathcal{K}_{h} w^{(n)}=f, \quad w^{(0)}=f .
$$

The following theorem gives the convergence result for this scheme.

Main Theorem. Suppose an initial state is prescribed, such that the exact equations of motion (1.1), (1.2) have a smooth solution for $0 \leq t \leq T$ meeting the conditions above (1.13). Let the numerical scheme be as presented, with the discrete derivative $D_{h, \nu}$ chosen according to (1.14)-(1.16) and the operator $P_{h}$ as in (1.32), (1.33). Let $G_{h}$ be a regularized Green's function satisfying (2.1)-(2.3), (2.9) and $\hat{G}_{h}<0$. Then for $h$ sufficiently small, the scheme has a solution with the prescribed initial state for $0 \leq t \leq T$, and in particular the discrete integral equation (1.25) can be solved. For each time $t \leq T$, the surface computed at the grid points $\alpha_{j}$ differs from the exact surface by $O\left(h^{3}\right)$ in $L_{h}^{2}$. The computed velocity potential at the surface has error $O\left(h^{3}\right)$ in $L_{h}^{2}$, and the computed velocity at the surface has error $O\left(h^{2}\right)$ in $L_{h}^{2}$.

This scheme is semidiscrete, i.e., discrete in space but not time. We expect the time integration to be done using an ODE solver such as the Runge-Kutta or Adams-Bashforth methods. Higher order versions of this scheme are possible but would require more complicated correction terms. We do not deal in detail here with the efficient computation of the discrete integrals; it is important to note, however, that with $n=N^{2}$ points to be tracked, the computation can be done in essentially $O(n)$ operations per time step. The four integrals can be put in matrixvector form, where the matrix comes from either $G_{h}^{\pi}$ or its gradient. There are two ways to proceed, based on the fast multipole method of Greengard and Rokhlin 14] or Ewald summation. Both have been used for doubly periodic water wave computations [4, 17]. We can choose the regularization of the Green's function, e.g., (2.19), so that it is significant only at distance $O(h)$ from the singularity. Then fast summation can be used except for $O(1)$ terms per integral, or $O(n)$ terms in total. An alternative is to adapt the fast version of Ewald summation of Strain [29]. 
As usual, the theorem asserts the convergence of the scheme as long as a smooth solution exists. Recently Sijue Wu has proved that a smooth solution of the initial value problem for water waves exists for a time depending on the initial state, in a three-dimensional fluid of infinite extent, tending to equilibrium at infinity [35]. (Earlier results were two-dimensional or assumed analytic data.) It is reasonable to expect that a result similar to Wu's holds for the present case of doubly periodic motion. Wu shows in her case that the pressure gradient at the surface is bounded away from zero,

$$
c=-\nabla p \cdot n \geq c_{0}>0,
$$

where $n$ is the normal outward from the fluid region. Such a result was proved for a bounded domain in [2]. The same condition holds in the present case; this can be seen as in $\S 4$ of [35]. The essential reason is that $-p$ is subharmonic and therefore obeys the strict maximum principle. The coefficient (1.35) appears in (1.4). The positivity is important for the well-posedness of the exact equations and consequently also for the numerical stability of the scheme under consideration, as seen in $\$ 6$ It means that the motion is well-posed even after a wave overturns. (Of course the model ceases to apply once the wave crashes.) Further discussion of the significance of (1.35) can be found in 5.

In analyzing the stability of the scheme we need to understand the mapping properties of the discrete integral operators applied to error terms. It is natural to view them as discrete versions of pseudodifferential operators; the important parts can be regarded as convolutions and estimated in the Fourier transform. This point of view is especially helpful in establishing the positivity of the operator $\Lambda$ using the assumption $\hat{G}_{h}<0$. In $\$ 4$ we develop some basic properties of discrete pseudodifferential operators under mild conditions, including a version of Gårding's inequality. In [25] general results for such operators were derived assuming a high wavenumber cut-off. Here we avoid this assumption and derive more limited results. In $\$ 5$ we show that various discrete operators related to $G_{h}^{\pi}$ are bounded, or gain derivatives, as linear operators on $L_{h}^{2}$. These are discrete versions of standard properties of single and double layer potentials. In the stability estimates of 4 we use these mapping properties to identify the principal error terms and simplify them, without treating less important terms in detail. The singularity subtraction helps to prevent spurious terms from appearing in the stability analysis. In fact, a scheme in 2-D like that of [6] can be shown to converge if singularity subtraction is used, without smoothing the points $x_{j}$ inside the integrals, as was done in [6].

We briefly outline the contents of the remaining sections. In $\$ 2$ we describe the regularized Green's function $G_{h}$ and derive needed formulas, construct the periodic version $G_{h}^{\pi}$, and prove that the error from replacing $G^{\pi}$ with $G_{h}^{\pi}$ in a single or double layer potential is $O\left(h^{3}\right)$. In $\S 3$ we analyze the quadrature error for the trapezoidal rule applied to singular integrals with regularization, and derive a formula to remove the largest error by local correction. We apply these results to the discrete integrals of (1.26), (1.27). In $\$ 4$ we prove basic properties of discrete pseudodifferential operators, and in $\$ 5$ the boundedness properties of discrete integral operators. The convergence of the scheme is proved in 6 , stability estimates are obtained for the computed velocity, using the results of $\$ \$ 4$ and 5 , a simplified equation is found for the growth of the error; and estimates are found for the rate of growth. Some arguments needed for the proof are deferred to $\$ 7$ 
The operator $\Lambda$ is essentially a convolution with $\nabla^{T} G$, following a derivative; see Lemma 6.8 and (6.31) for the discrete version. The positivity of the discrete $\Lambda$ depends on the choice of quadrature for the singular integral, as well as the choice of the two derivatives. It appears difficult to maintain this condition in discretizing the integral without regularizing $G$; the sign condition on $\hat{G}_{h}$ and the matching of the two derivatives ensure the positivity here. In the stability argument of $\S[6$ the positivity of $\Lambda$ is needed because it enters the energy estimates; see (6.55). We also need the discrete $\Lambda$ to dominate the discrete first derivative, i.e., the derivative can be written as a bounded operator times $\Lambda$ (see (6.49)-(6.50). It is for this latter reason that we require the derivative in (1.14) to have nonzero symbol $\sigma$ for $k \neq 0$; if $\sigma$ decays in the high wavenumbers, then the symbol of $\Lambda$ decays faster, since $\Lambda$ has two derivatives. In the 2-D convergence argument of [6], the analogue of $\nabla^{T} G$ was the Hilbert transform $H$; the discrete $H$ given by alternate quadrature had symbol with modulus one, and the two needed properties of $\Lambda=H D$ followed directly.

\section{The Regularized Green's Function}

In this section we describe the class of regularized Green's functions and find the Fourier transform of the restriction to a plane in terms of the 3-D transform. We need to compute this transform explicitly for the correction terms in the integral equation (1.25). We find a specific $G_{h}$, based on the Gaussian function, which has all the needed properties. We construct the periodic Green's function as a sum of images, adjusted by constants. Finally, we prove (Theorems 2.2] and 2.3) that the error in a single or double layer potential due to the regularization is $O\left(h^{3}\right)$.

The regularized Green's function $G_{h}$ will have the form

$$
G_{h}(x)=-(4 \pi r)^{-1} s(r / h), \quad r=|x|,
$$

with $s$ chosen so that $s(r) \rightarrow 1$ rapidly as $r \rightarrow \infty$ and $G_{h}$ is smooth. A convenient assumption for the decay of $s-1$ is

$$
\left|D_{r}^{k}(s(r)-1)\right| \leq C r^{-4-k}, \quad r \geq 1 .
$$

For $G_{h}$ to be smooth, we need $s(|x|) /|x|$ to be smooth as a function of $x$, and thus as a function of $|x|^{2}$; i.e., we assume

$$
s(r) / r \text { is a smooth function of } r^{2}, \quad 0 \leq r<\infty .
$$

Then for $h>0, G_{h}(x)=h^{-1} G_{1}(x / h)$. For later use we note that (cf. [1])

$$
\left|D_{x}^{k} G_{h}(x)\right| \leq \begin{cases}C|x|^{-|k|-1}, & |x| \geq h, \\ C h^{-|k|-1}, & |x| \leq h .\end{cases}
$$

Letting $\psi=\Delta G_{1}$, we find that

$$
\psi(x)=\Delta G_{1}(x)=-(4 \pi r)^{-1} s^{\prime \prime}(r), \quad r=|x|,
$$

and correspondingly

$$
\Delta G_{h}(x)=\psi_{h}(x) \equiv h^{-3} \psi(|x| / h),
$$

which approximates the delta function as $h \rightarrow 0$.

For accuracy we need a further condition on $s$. It is well-known (e.g., [16]) that the error from replacing $G$ by $G_{h}$ in an integral is determined by moments of $s-1$ 
or $\psi$. Here we are interested in integrals on a surface, and so we consider, for a homogeneous polynomial $p_{n}(x)$ of degree $n$, the integral

$$
\int_{\mathbf{R}^{2}}\left(G_{h}(x)-G(x)\right) p_{n}(x) d x=-\frac{1}{4 \pi} \int_{\mathbf{R}^{2}}(s(|x| / h)-1)|x|^{-1} p_{n}(x) d x,
$$

which becomes in polar coordinates

$$
C_{n} \int_{0}^{\infty}(s(r / h)-1) r^{n} d r=C_{n} h^{n+1} \int_{0}^{\infty}(s(\rho)-1) \rho^{n} d \rho .
$$

The largest error is $O(h)$ with $n=0$. For this reason we require of $s$ that

$$
\int_{0}^{\infty}(s(r)-1) d r=0 \text {. }
$$

For odd $n$, the error terms above are zero since the integrand is odd. Thus with condition (2.9) we expect the error from the regularization to be $O\left(h^{3}\right)$. This is verified for single layer potentials in Theorem 2.2. Given an arbitrary $s_{(1)}$ satisfying $(2.2),(2.3)$, we can easily produce a related function satisfying (2.9), as well as (2.2), (2.3), by setting

$$
s_{(3)}(r)=s_{(1)}(r)+r \partial_{r} s_{(1)}(r)
$$

Condition (2.9) can be verified for $s_{(3)}$ by an integration by parts. The corresponding relationship for the $\psi^{\prime}$ 's is $\psi_{(3)}=4 \psi_{(1)}+r \partial_{r} \psi_{(1)}$. (A similar strategy for satisfying moment conditions was used in [8].)

When $G_{h}$ has the form (2.1), we have a similar expression for $\nabla G_{h}$,

$$
\nabla G_{h}(x)=\frac{x}{4 \pi r^{3}} \tilde{s}(r / h), \quad \tilde{s}(r)=s(r)-r \partial_{r} s(r) .
$$

Here $\tilde{s}$ has the same properties $(2.2),(2.3)$ as $s$. If $s$ satisfies the moment condition (2.9), then the same holds for $\tilde{s}$; this can be seen by an integration by parts.

We need explicit formulas for $\hat{G}_{h}$, the Fourier transform of $G_{h}$ in $\mathbf{R}^{3}$, and for the transform of $G_{h}$ on planes, since we work with integrals on surfaces. We will write the Fourier transform of a function $f$ on $\mathbf{R}^{d}$ as

$$
\hat{f}(k)=(2 \pi)^{-d / 2} \int f(x) e^{-i k x} d x, \quad f(k)=(2 \pi)^{-d / 2} \int \hat{f}(k) e^{i k x} d k .
$$

The hypotheses imply that $\hat{G}_{h}(k)$ is smooth for $k \neq 0$ and decays rapidly for large $k$. More particularly, it will be important to require that $\hat{G}_{h}(k)<0$. Since $-|k|^{2} \hat{G}(k)=\hat{\psi}(k)$, this is equivalent to requiring that $\hat{\psi}(k)>0$. To find the transform of the restriction to a plane, we first identify $\left(x_{1}, x_{2}\right) \in \mathbf{R}^{2}$ with $\left(x_{1}, x_{2}, 0\right) \in$ $\mathbf{R}^{3}$. Then the Fourier transform of the function on $\mathbf{R}^{2}$ given by $x \rightarrow G_{h}(x, 0)$ is

$$
G_{1}(\cdot, 0)^{\wedge}\left(k_{1}, k_{2}\right)=(2 \pi)^{-1 / 2} \int_{-\infty}^{\infty} \hat{G}_{1}\left(k_{1}, k_{2}, k_{3}\right) d k_{3} .
$$

Since $\hat{G}_{1}$ is radial, this depends only on $\left|\left(k_{1}, k_{2}\right)\right|$, and we write $G_{1}(\cdot, 0)^{\wedge}\left(k_{1}, k_{2}\right)=$ $\Gamma\left(\left|\left(k_{1}, k_{2}\right)\right|\right)$, with $\Gamma$ defined by

$$
\Gamma(\rho)=(2 \pi)^{-1 / 2} \int_{-\infty}^{\infty} \hat{G}_{1}(\rho, 0, \ell) d \ell .
$$

More generally we will use the transform of $G_{1} \circ J: \mathbf{R}^{2} \rightarrow \mathbf{R}$, given by $\alpha \rightarrow G_{1}(J \alpha)$, where $J: \mathbf{R}^{2} \rightarrow \mathbf{R}^{3}$ is a $3 \times 2$ matrix. In our applications $J$ will be the Jacobian 
matrix of a coordinate mapping. Since $G_{1}$ is radial, $G_{1}(J \alpha)$ is a function of $|J \alpha|^{2}=$ $\left|J^{*} J \alpha \cdot \alpha\right|^{2}=|B \alpha|^{2}$, where $B=\left(J^{*} J\right)^{1 / 2}$. Thus $G_{1} \circ J(\alpha)=G_{1}(B \alpha, 0)$, and

$$
\left(G_{1} \circ J\right)^{\wedge}(k)=(\operatorname{det} B)^{-1} G_{1}(\cdot, 0)^{\wedge}\left(B^{-1} k\right), \quad k \in \mathbf{R}^{2}-\{0\} .
$$

We can now combine this with (2.13), using $\left|B^{-1}(k)\right|=\left|\left(J^{*}\right)^{-1} k\right|$, to obtain

$$
\left(G_{1} \circ J\right)^{\wedge}(k)=\left(\operatorname{det} J^{*} J\right)^{-1 / 2} \Gamma\left(\left|\left(J^{*}\right)^{-1} k\right|\right), \quad k \in \mathbf{R}^{2}-\{0\} .
$$

If the two columns of $J$ are vectors $X_{1}, X_{2} \in \mathbf{R}^{3}$, then $\left(J^{*} J\right)_{\mu \nu}=X_{\mu} \cdot X_{\nu}=g_{\mu \nu}$, the usual metric induced in the coordinate plane. Also $\left|\left(J^{*}\right)^{-1} k\right|^{2}=g^{\mu \nu} k_{\mu} k_{\nu}$, summed over $\mu, \nu$, where $\left(g^{\mu \nu}\right)=\left(g_{\mu \nu}\right)^{-1}$. Thus the formula above becomes

$$
\left(G_{1} \circ J\right)^{\wedge}(k)=\left(\operatorname{det} g_{\mu \nu}\right)^{-1 / 2} \Gamma\left(\sqrt{g^{\mu \nu} k_{\mu} k_{\nu}}\right), \quad k \in \mathbf{R}^{2}-\{0\} .
$$

Of course $\left(G_{1} \circ J\right)^{\wedge}(k)<0$ since $\hat{G}_{1}<0$; this will be important later.

We now describe a specific choice of $G_{h}$ which meets all our requirements. We start with the error function

$$
\operatorname{erf}(r)=\frac{2}{\sqrt{\pi}} \int_{0}^{r} e^{-s^{2}} d s .
$$

It fulfills conditions (2.2), (2.3). Using (2.10), we define $s(r)=\operatorname{erf}(r)+r \operatorname{erf}^{\prime}(r)$ or

$$
s(r)=\operatorname{erf}(r)+2 \pi^{-1 / 2} r e^{-r^{2}}
$$

so that $s$ satisfies the moment condition (2.9). For this $s$ we find

$$
\begin{gathered}
\tilde{s}(r)=\operatorname{erf}(r)+2 \pi^{-1 / 2}\left(2 r^{3}-r\right) e^{-r^{2}}, \\
\psi(r)=\pi^{-3 / 2}\left(4-2 r^{2}\right) e^{-r^{2}} .
\end{gathered}
$$

As for the transform, we have

$$
\hat{G}_{1}(k)=-(2 \pi)^{-3 / 2}\left(\frac{1}{2}+|k|^{-2}\right) e^{-|k|^{2} / 4}, \quad k \in \mathbf{R}^{3} .
$$

Then, from the above,

$$
\Gamma(\rho)=-(2 \pi)^{-2} \int_{-\infty}^{\infty}\left(\frac{1}{2}+\frac{1}{\rho^{2}+\ell^{2}}\right) e^{-\left(\rho^{2}+\ell^{2}\right) / 4} d \ell .
$$

Using [13], formula 3.466, we have

$$
\Gamma(\rho)=-(2 \pi)^{-2}\left(\sqrt{\pi} e^{-\rho^{2} / 4}+\frac{\pi}{\rho} \operatorname{erfc}(\rho / 2)\right),
$$

where erfc $=1-$ erf. We could decide to use $s(|x| / a h)$ instead of $s(|x| / h)$ in the definition of $G_{h}$, where $a>0$ is some constant. The effect would be to replace $G_{h}$ by $G_{a h}$. Then $\left(G_{1} \circ J\right)$ would be replaced by $\left(G_{a} \circ J\right)$, and (2.17) by

$$
\left(G_{a} \circ J\right)^{\wedge}(k)=a\left(\operatorname{det} g_{\mu \nu}\right)^{-1 / 2} \Gamma(a \rho), \quad \rho=\sqrt{g^{\mu \nu} k_{\mu} k_{\nu}} .
$$

The condition $\hat{G}_{h}<0$ is evident in (2.22) for the specific choice of $G_{h}$ given by (2.19). The condition would hold for a variety of other choices as well. Suppose we first choose a smooth delta function as in (2.6) by taking $\psi_{(1)}$ to be a smooth, rapidly decreasing, radial function, with integral one, such that $\hat{\psi}_{(1)}(k)>0$ and $\hat{\psi}_{(1)}$ decreases with $|k|$. If we define $s_{(1)}$ by (2.5), $s_{(3)}$ by (2.10), and $G_{h}$ by (2.1) using $s_{(3)}$, it follows that the needed conditions hold, including $\hat{G}_{h}<0$. 
We next discuss the periodic versions of the Green's function and its regularization. Because of the slow decay at infinity, we write the sum with a reflection and with a constant subtracted from each term. We define

$$
G^{\pi}(x)=\frac{1}{2} \sum_{n \in \mathbf{Z}^{2}}^{\prime}\left(G(x-2 \pi(n, 0))+G(x+2 \pi(n, 0))+\frac{1}{2 \pi|n|}\right),
$$

where the prime on the sum means that $1 /|n|$ is omitted at $n=0$. The gradient is

$$
\nabla G^{\pi}(x)=\frac{1}{2} \sum_{n \in \mathbf{Z}^{2}}(\nabla G(x-2 \pi(n, 0))+\nabla G(x+2 \pi(n, 0))),
$$

and we define $G_{h}^{\pi}, \nabla G_{h}^{\pi}$ similarly.

Lemma 2.1. The sums for $G_{h}^{\pi}(x)$ and $\nabla G_{h}^{\pi}(x)$ converge uniformly on bounded sets for fixed $h$, and those for $G^{\pi}(x)$ and $\nabla G^{\pi}(x)$ converge in $L^{1}$ on bounded sets. $G^{\pi}(x)$ and $G_{h}^{\pi}(x)$ are periodic and satisfy

$$
\Delta G^{\pi}(x)=\sum_{n} \delta(x+2 \pi(n, 0)), \quad \Delta G_{h}^{\pi}(x)=\sum_{n} \psi_{h}(x+2 \pi(n, 0)) .
$$

Proof. For fixed $x$ and large $y$, we have

$$
G(x+y)=-\frac{1}{4 \pi}\left(\frac{1}{|y|}-\frac{y \cdot x}{|y|^{3}}+O\left(|y|^{-3}\right) \quad \text { as } y \rightarrow \infty .\right.
$$

If we add $G(x \pm y)$, the second term above is canceled, and so with $y= \pm 2 \pi(n, 0)$, we see that the $n$th term in (2.26) is $O\left(|n|^{-3}\right)$. Thus the sum for $G^{\pi}(x)$ converges pointwise away from the singularity, and since the singularity is integrable, the sum also converges in $L^{1}$ on a bounded set. For the regularized version $G_{h}$, we note that

$$
\left|G_{h}(x \pm 2 \pi(n, 0))-G(x \pm 2 \pi(n, 0))\right|=O\left(|n|^{-5}\right) \quad \text { as } n \rightarrow \infty
$$

because of (2.2). Thus the sum for $G_{h}^{\pi}$ converges similarly to $G^{\pi}$, but $G_{h}^{\pi}$ is bounded for fixed $h$, so that the convergence is uniform on bounded sets. It is correct to apply $\Delta$ termwise in the distributional sense, so that both equations (2.28) hold as distributions. However, since $\Delta G_{h}^{\pi}$ is smooth, the second case is true in the classical sense as well. Similar considerations apply to the gradients.

It remains to verify that $G^{\pi}$ and $G_{h}^{\pi}$ are actually periodic. Specifically, let $x$ be a point which is not a periodic image of 0 ; we verify that $G^{\pi}(x+(2 \pi, 0,0))=$ $G^{\pi}(x)$. We will use the notation $r(n)=|n|^{-1}$ if $n \neq 0, r(0)=0$, and also $n^{+}=$ $\left(n_{1}+1, n_{2}, 0\right), n^{-}=\left(n_{1}-1, n_{2}, 0\right)$. Let $B_{M}=\left\{\left(n_{1}, n_{2}\right) \in \mathbf{R}^{2}:\left|n_{j}\right| \leq M, j=1,2\right\}$. Then

$$
-8 \pi G^{\pi}(x+(2 \pi, 0,0))=\lim _{M \rightarrow \infty} \sum_{B_{M}}\left(\left|x+2 \pi n^{+}\right|^{-1}+\left|x-2 \pi n^{-}\right|^{-1}-2 r(n)\right) .
$$

We will show below that we can replace $2 r(n)$ above by $r\left(n^{+}\right)+r\left(n^{-}\right)$. If we do this, the sum over $B_{M}$ can be rewritten as

$$
\sum_{B_{M}}\left(\left|x+2 \pi n^{+}\right|^{-1}-r\left(n^{+}\right)\right)+\sum_{B_{M}}\left(\left|x-2 \pi n^{-}\right|^{-1}-r\left(n^{-}\right)\right) .
$$

In the first sum we can shift the $n^{+}$back to $n$, ranging over $B_{M}$, except for $O(M)$ terms, each of size $O\left(M^{-2}\right)$. Since $B_{M}$ is symmetric, $n \rightarrow-n$, this sum converges 
to $-4 \pi G^{\pi}(x)$ as $M \rightarrow \infty$. The same argument applies to the $n^{-}$term, and the desired equality is verified. The same argument works for $G_{h}^{\pi}$.

We still must show that the replacement above did not change (2.31); i.e., we must show that the absolutely convergent sum $\sum\left(r\left(n^{+}\right)+r\left(n^{-}\right)-2 r(n)\right)$ is zero. It is the limit as $M \rightarrow \infty$ of

$$
\sum_{B_{M}}\left(r\left(n^{+}\right)-r(n)\right)+\sum_{B_{M}}\left(r\left(n^{-}\right)-r(n)\right) .
$$

We can shift the index in the second sum and write it as the sum of $r(n)-r\left(n^{+}\right)$ over a set which is $B_{M}$, except for $O(M)$ terms each of size $O\left(M^{-2}\right)$. Within $B_{M}$, the terms cancel exactly, and therefore the expression above approaches 0 as $M \rightarrow \infty$.

Finally we estimate the error introduced by the regularization of the Green's function for single or double layer potentials on a doubly periodic surface.

Theorem 2.2. Let $\alpha \rightarrow x$ be a smooth mapping from $\mathbf{R}^{2}$ to $\mathbf{R}^{3}$ so that $x(\alpha)-(\alpha, 0)$ is doubly periodic with period $2 \pi$ and (1.13) is satisfied. Let $f$ be a smooth periodic function of $\alpha, G^{\pi}$ the periodic Green's function, and $G_{h}^{\pi}$ the regularized version. Then for any $\alpha_{0}$

$$
\left|\int G_{h}^{\pi}\left(x\left(\alpha_{0}\right)-x(\alpha)\right) f(\alpha) d \alpha-\int G^{\pi}\left(x\left(\alpha_{0}\right)-x(\alpha)\right) f(\alpha) d \alpha\right| \leq C h^{3},
$$

where the integral is over a period square in $\alpha$.

Proof. We can take the domain of integration to be

$$
B_{0}=\left\{\alpha=\left(\alpha_{1}, \alpha_{2}\right) \in \mathbf{R}^{2}:\left|\alpha_{\nu}-\left(\alpha_{0}\right)_{\nu}\right|<\pi, \nu=1,2\right\} .
$$

To separate out the singularity, we introduce a cut-off function $\zeta$ on $\mathbf{R}^{2}$ so that $\zeta(\alpha)=1$ near $\alpha=0$ and $\zeta(\alpha)=0$ for $|\alpha|>\pi / 2$. Now define, for all $\alpha$,

$$
G_{h 0}^{\pi}(\alpha)=\sum_{n \in \mathbf{Z}^{2}} G_{h}\left(x\left(\alpha_{0}\right)-x(\alpha)-2 \pi(n, 0)\right) \zeta\left(\alpha_{0}-\alpha-2 \pi n\right) .
$$

Then $G_{h 0}^{\pi}$ is periodic in $\alpha$, since adding a period to $\alpha$ only shifts the sum; only one term can be nonzero. Similarly we define $G_{0}^{\pi}$ from $G^{\pi}$ using the same $\zeta$. Let $G_{h \infty}^{\pi}=G_{h}^{\pi}-G_{h 0}^{\pi}$ and $G_{\infty}^{\pi}=G^{\pi}-G_{0}^{\pi}$, as functions of $\alpha$. Now $G_{h \infty}^{\pi}$ and $G_{\infty}^{\pi}$ are smooth and periodic. We first argue that their difference contributes little to the integral. From (2.2), we have

$$
\left|G_{h}\left(x\left(\alpha_{0}\right)-x(\alpha)\right)-G\left(x\left(\alpha_{0}\right)-x(\alpha)\right)\right| \leq C h^{4}\left|x\left(\alpha_{0}\right)-x(\alpha)\right|^{-5}
$$

provided $\left|x\left(\alpha_{0}\right)-x(\alpha)\right|$ is bounded away from zero. By (1.13), the latter is true if $\left|\alpha_{0}-\alpha\right|$ is bounded away from zero, as it is on the support of $1-\zeta$. The same is true with $\alpha$ replaced by $\alpha+2 \pi n$. Consequently $G_{h \infty}^{\pi}-G_{\infty}^{\pi}$ is $O\left(h^{4}\right)$, uniformly in $\alpha$; the $n$th term decays like $|n|^{-5}$, so that the sum of estimates converges. Thus the integral corresponding to the difference $G_{h \infty}^{\pi}-G_{\infty}^{\pi}$ is also $O\left(h^{4}\right)$. We are now reduced to the case where $G_{h}^{\pi}$ and $G^{\pi}$ are replaced by $G_{h 0}^{\pi}$ and $G_{0}^{\pi}$. We note that for $\alpha \in B_{0}$ we have $\left|\alpha_{0}-\alpha \pm 2 \pi n\right|>\pi$ for $n \neq 0$, so that $\zeta=0$ there. Consequently $G_{h 0}^{\pi}(\alpha)=G_{h}\left(x\left(\alpha_{0}\right)-x(\alpha)\right) \zeta\left(\alpha_{0}-\alpha\right)$ for $\alpha \in B_{0}$, and similarly for $G^{\pi}$.

It remains to obtain a local estimate near the singularity at $\alpha_{0}$. For convenience we assume from now on that $\alpha_{0}=0$ and $x\left(\alpha_{0}\right)=0$. We also may as well assume 
that $f(\alpha)$ has support near $\alpha=0$, because of the $\zeta$ factor. The proof now amounts to showing that

$$
\left|\int_{\mathbf{R}^{2}}\left(G_{h}(x(\alpha))-G(x(\alpha))\right) f(\alpha) d \alpha\right| \leq C h^{3}
$$

The integral is

$$
\left.-\int_{\mathbf{R}^{2}}(s(r / h)-1)\right)\left(\frac{1}{4 \pi r}\right) f(\alpha) d \alpha, \quad r=|x(\alpha)| .
$$

Let $(\rho, \theta)$ be polar coordinates for $\alpha$; the integral becomes

$$
\left.-(4 \pi)^{-1} \int_{0}^{2 \pi} \int_{0}^{\infty}(s(r / h)-1)\right) \frac{\rho}{r} f(\alpha) d \rho d \theta
$$

We will change variables in the inner integral from $\rho$ to $r$. First we write the Taylor formula for $x(\alpha)$ as $x(\alpha)=J \alpha+q(\alpha)+O\left(\alpha^{3}\right)$, where $J=(\partial x / \partial \alpha)(0)$ and $q$ is bilinear. As a consequence we can write

$$
r / \rho=|x(\alpha)| /|\alpha|=a(\theta)+b(\theta) \rho+\gamma(\rho, \theta) \rho^{2},
$$

where $\gamma$ is smooth in $(\rho, \theta)$, regarded as independent variables. Here

$$
a(\theta)=|J \alpha| /|\alpha|, \quad b(\theta)=J \alpha \cdot q(\alpha) /|\alpha|^{2}|J \alpha| .
$$

For later use we note that $a$ is even and $b$ is odd in $\alpha$, i.e., $a(\theta+\pi)=a(\theta)$, $b(\theta+\pi)=-b(\theta)$. Assuming the support of the cut-off function $\zeta$ was chosen small enough, we can invert (2.41) to obtain

$$
\rho / r=\tilde{a}(\theta)+\tilde{b}(\theta) r+\tilde{\gamma}(r, \theta) r^{2},
$$

where $\tilde{a}=1 / a, \tilde{b}=-b / a^{3}$, so that $\tilde{a}, \tilde{b}$ have the same parity as $a, b$, and $\tilde{\gamma}(r, \theta)$ is smooth. We now convert the $\rho$-integral above to

$$
\left.\int_{0}^{\infty}(s(r / h)-1)\right) \frac{\rho}{r} \frac{\partial \rho}{\partial r} f(\alpha) d r .
$$

Only the low powers of $r$ will matter, and we use Taylor expansions. We have $\rho / r=\tilde{a}+\tilde{b} r+O\left(r^{2}\right)$ and $\partial \rho / \partial r=\tilde{a}+2 \tilde{b} r+O\left(r^{2}\right)$. We can write the expression for $f(\alpha)$ as $f=f_{0}+f_{1}(\theta) \rho+O\left(\rho^{2}\right)$, with $f_{1}(\theta)$ odd. Converting from $\rho$ to $r$, we have $f=f_{0}+\tilde{a} f_{1} r+O\left(r^{2}\right)$. Multiplication of these expressions gives

$$
(\rho / r)(\partial \rho / \partial r) f(\alpha)=\tilde{a}^{2} f_{0}+\left(3 \tilde{a} \tilde{b} f_{0}+\tilde{a}^{3} f_{1}\right) r+\tilde{f}(r, \theta) r^{2}
$$

with $\tilde{f}$ smooth in $r, \theta$. When we substitute this into the integral, the constant term contributes zero because of the moment condition (2.9). The term linear in $r$ has a coefficient which is odd, so that it also contributes zero after integration in $\theta$. The remaining part of the $r$-integral is now

$$
\left.\left.\int_{0}^{\infty}(s(r / h)-1)\right) \tilde{f}(r, \theta) r^{2} d r=h^{3} \int_{0}^{\infty}(s(r / h)-1)\right) \tilde{f}(r, \theta)(r / h)^{2} d(r / h) .
$$

The last integral is bounded uniformly in $h$, since $\tilde{f}$ is bounded and $s-1$ decreases rapidly. The total error has been reduced to the last expression and is therefore $O\left(h^{3}\right)$, as claimed.

Next we have the analogue of Theorem 2.2 for double layer potentials. 
Theorem 2.3. Let $\alpha \rightarrow x$ be the mapping of Theorem [2.2, and let $F(\alpha)$ be a smooth vector-valued function of $\alpha$. Assume that for some $\alpha_{0}$ the vector $F\left(\alpha_{0}\right)$ is parallel to $n_{0}$, the normal to the surface at $x\left(\alpha_{0}\right)$. Then

$$
\left|\int \nabla G_{h}^{\pi}\left(x\left(\alpha_{0}\right)-x(\alpha)\right) \cdot F(\alpha) d \alpha-\int \nabla G^{\pi}\left(x\left(\alpha_{0}\right)-x(\alpha)\right) \cdot F(\alpha) d \alpha\right| \leq C h^{3},
$$

where the integral is over a period square in $\alpha$.

Proof. As in Theorem 2.2, we can reduce the estimate to the case where $\alpha_{0}=0$, $x\left(\alpha_{0}\right)=0$, and $F$ is supported in a small neighborhood of $\alpha=0$. We then need to show that

$$
\left|\int_{\mathbf{R}^{2}}\left(\nabla G_{h}(x(\alpha))-\nabla G(x(\alpha))\right) \cdot F(\alpha) d \alpha\right| \leq C h^{3} .
$$

The integral is proportional to

$$
\left.\int_{\mathbf{R}^{2}}(\tilde{s}(r / h)-1)\right) r^{-3} x(\alpha) \cdot F(\alpha) d^{2} \alpha, \quad r=|x(\alpha)| .
$$

Proceeding as before, we obtain the $r$-integral

$$
\left.\int_{0}^{\infty}(\tilde{s}(r / h)-1)\right) x(\alpha) \cdot F(\alpha) r^{-3} \rho \frac{\partial \rho}{\partial r} d r
$$

Expanding $x$ as $x(\alpha)=J \alpha+q(\alpha)+O\left(\rho^{3}\right)$ and $F(\alpha)=F_{0}+F_{1} \rho+O\left(\rho^{2}\right)$, and noting that $J \alpha \cdot F_{0}=0$, we have

$$
x \cdot F(\alpha)=c_{2}(\theta) \rho^{2}+c_{3}(\theta) \rho^{3}+O\left(\rho^{4}\right)
$$

with $c_{2}$ even and $c_{3}$ odd. Now using $\rho=\tilde{a} r+\tilde{b} r^{2}+O\left(r^{3}\right)$, we get

$$
x \cdot F=c_{2} \tilde{a}^{2} r^{2}+\left(c_{3} \tilde{a}^{3}+2 \tilde{a} \tilde{b}\right) r^{3}+O\left(r^{4}\right) .
$$

Multiplying out, we find that

$$
(x \cdot F) r^{-3} \rho(\partial \rho / \partial r)=\tilde{a}^{4} c_{2}+\left(\tilde{a}^{5} c_{3}+2 \tilde{a}^{3} \tilde{b} c_{2}+3 \tilde{a}^{3} \tilde{b} c_{2}\right) r+O\left(r^{2}\right) .
$$

The coefficient of $r$ is odd, and the argument proceeds just as before.

\section{QuADRATURE OF SINGULAR INTEGRALS}

In this section we develop a general approach to quadrature for singular integrals with regularization. We find an expansion in powers of $h$ for the error in the trapezoidal rule, similar to that of Lyness [23] and Goodman et al. [12] without regularization, adapting the approach of [12]. We show that the largest error can be identified in terms of the Fourier transform of the regularized kernel using the Poisson summation formula. A correction can then be found to improve the order of accuracy. The application to single and double layer potentials is given in Theorem 3.7. We derive the correction terms in (1.26), (1.27) and prove that the quadrature errors in these equations are $O\left(h^{3}\right)$. For unregularized singular integrals, corrections are more difficult to find, but methods have been developed in [22], [17].

For later reference, we state the Poisson summation formula for a smooth, rapidly decreasing function $f$ on $\mathbf{R}^{d}$ :

$$
(2 \pi)^{-d / 2} \sum_{j \in Z^{d}} f(j h) e^{-i k j h} h^{d}=\sum_{n \in Z^{d}} \hat{f}(k+2 \pi n / h)
$$


We will need a general estimate for quadrature of mildly regular functions. The following statement was proved in [11], [1] for $F$ independent of $h$. The proof of Lemma 2.2 in [1] is direct from the Poisson formula.

Lemma 3.1. Let $F(x)$ be a function of $x$ in $\mathbf{R}^{d}$ such that $D_{x}^{\beta} F$ is in $L^{1}\left(\mathbf{R}^{d}\right)$ for multi-indices $\beta$ with $|\beta|=\ell$, where $\ell \geq d+1$. Then, with a universal constant $C_{\ell}$,

$$
\left|\sum_{n \in Z^{d}} F(n h) h^{d}-\int_{\mathbf{R}^{d}} F(x) d x\right| \leq C_{\ell} h^{\ell} \sum_{|\beta|=\ell}\left|D_{x}^{\beta} F\right|_{L^{1}} .
$$

We begin with a treatment of quadrature errors for singular integrals, regularized on the scale of the grid size. We start with a singular kernel $K(x)$, defined for nonzero $x$ in $d$-space $\mathbf{R}^{d}$, which is homogeneous of degree $m$ for integer $m$, i.e.,

$$
K(a x)=a^{m} K(x), \quad a>0, \quad x \neq 0 .
$$

We also work with modified kernels of the form $K_{h}(x)=K(x) s(x / h)$, where $s$ approaches 1 for large argument, and $s$ is chosen so that $K_{h}$ is smooth up to $x=0$. Note that $K_{h}(x)=h^{m} K_{1}(x / h)$. The following two lemmas will be fundamental. They are adapted from the proof of Lemma 1 in [12].

Lemma 3.2. Let $K$ and $s$ be smooth functions on $\mathbf{R}^{d}-\{0\}$ such that $K$ is homogeneous of degree $m$, in the sense of (3.3), and

$$
\left|D^{k} s(x)\right| \leq C|x|^{-|k|}, \quad|x| \geq 1 .
$$

For $h>0$, let $K_{h}(x)=K(x) s(x / h)$, and assume $K_{h}$ continues as a smooth function to $x=0$, so that $K_{h}(0)=h^{m} K_{1}(0)$. Finally, let $\zeta(x)$ be a smooth cut-off function with $\zeta(x) \equiv 1$ for $x$ in a neighborhood of 0 and $\zeta=0$ outside a bounded set. Now approximate the integral

$$
I=\int_{\mathbf{R}^{d}} K_{h}(x) \zeta(x) d x=\int_{\mathbf{R}^{d}} K(x) s(x / h) \zeta(x) d x
$$

by the sum of values at $n h$, where $n$ is a multi-integer:

$$
S=\sum_{n \in \mathbf{Z}^{d}} K_{h}(n h) \zeta(n h) h^{d}=\sum_{n \in \mathbf{Z}^{d}} K(n h) s(n) \zeta(n h) h^{d} .
$$

Then as $h \rightarrow 0$,

$$
S-I=c_{0} h^{d+m}+O\left(h^{\ell}\right),
$$

where $\ell$ is large depending on the smoothness of $K(x)$ and $s(x)$ for $|x| \geq 1$.

For example, if $m=1-d$, we have a first order error with a much smaller remainder. In some cases we can identify the constant $c_{0}$, as explained below. In the second expression for the sum $S$, the proper limiting value should be used for the $n=0$ term, namely $K_{1}(0) h^{d+m}$. The proof of the lemma below also shows that the quadrature error for the unmodified $K$, omitting the $n=0$ term, has the same behavior in case $m \geq 1-d$, as shown in [12]:

$$
\sum_{n \neq 0} K(n h) \zeta(n h) h^{d}-\int_{\mathbf{R}^{d}} K(x) \zeta(x) d x=c_{0}^{\prime} h^{d+m}+O\left(h^{\ell}\right) .
$$

In the regularized case, the $n=0$ term is $O\left(h^{d+m}\right)$, so that the conclusion is valid whether or not the $n=0$ term is included in the quadrature. 
Proof. We use a simplified version of the argument in [12, modified for the smoothing of the kernel. We can rewrite the integral $I$ using the substitution $y=x / h$ and the homogeneity of $K$ as

$$
I=h^{d+m} \mathcal{I}, \quad \mathcal{I} \equiv \int_{\mathbf{R}^{d}} K(y) s(y) \zeta(y h) d y .
$$

Similarly we can write the sum as

$$
S=h^{d+m} \mathcal{S}, \quad \mathcal{S} \equiv \sum_{n} K(n) s(n) \zeta(n h) .
$$

Now $h$ appears inside $\mathcal{I}$ and $\mathcal{S}$ only in $\zeta$. As in [12] we differentiate in $h$ and compare the resulting sum and integral. We find that

$$
\mathcal{I}^{\prime}(h)=\int_{\mathbf{R}^{d}} K(y) s(y) y \cdot \nabla \zeta(y h) d y=h^{-(d+m+1)} \int_{\mathbf{R}^{d}} K(x) s(x / h) x \cdot \nabla \zeta(x) d x
$$

and

$$
\mathcal{S}^{\prime}(h)=\sum_{n} K(n) s(n) n \cdot \nabla \zeta(n h)=h^{-(d+m+1)} \sum_{n} K(n h) s(n)(n h) \cdot \nabla \zeta(n h) \cdot h^{d} .
$$

The integrand and summand are nonzero only on the support of $\nabla \zeta$. We take this support to be $\left\{x: r_{1} \leq|x| \leq r_{2}\right\}$ for some $r_{2}>r_{1}>0$. We can regard the last sum as a trapezoidal rule approximation to the integral above. To estimate the error using Lemma 3.1 we need to estimate the derivatives of the integrand. Since $|x| \geq r_{1}$, derivatives of $K$ are uniformly bounded. Moreover, from 3.4 we have

$$
\left|D_{x}^{k}(s(x / h))\right| \leq C|x|^{-|k|}, \quad|x| \geq h .
$$

Thus the $L^{1}$ norm of each derivative of the integrand is bounded uniformly in $h$, up to some order limited by the smoothness of $K$ and $s$. Then, by Lemma 3.1

$$
\left|\mathcal{S}^{\prime}(h)-\mathcal{I}^{\prime}(h)\right| \leq C_{\ell} h^{\ell-d-m-1}, \quad h>0,
$$

for large $\ell$. Now $\mathcal{S}(h)-\mathcal{I}(h)$ is smooth for $h>0$ and has a limit at $h=0$ provided $\ell$ is large enough. Then

$$
(\mathcal{S}-\mathcal{I})(h)=(\mathcal{S}-\mathcal{I})(0)+\int_{0}^{h}\left(\mathcal{S}^{\prime}-\mathcal{I}^{\prime}\right)=c_{0}+O\left(h^{\ell-d-m}\right)
$$

for some constant $c_{0}$, so that $S(h)-I(h)=c_{0} h^{d+m}+O\left(h^{\ell}\right)$, as claimed.

Lemma 3.3. Let $K, s, K_{h}, m, \zeta$ be as in the previous lemma, and let $f(x)$ be a smooth function on $\mathbf{R}^{d}$ such that $f$ and its derivatives are rapidly decreasing. Assume that $K, s$ and $\zeta$ are even in $x$. Then the integral

$$
I=\int_{\mathbf{R}^{d}} K_{h}(x) f(x) d x=\int_{\mathbf{R}^{d}} K(x) s(x / h) f(x) d x
$$

is approximated by the sum

$$
S=\sum_{n \in \mathbf{Z}^{d}} K_{h}(n h) f(n h) h^{d}=\sum_{n \in \mathbf{Z}^{d}} K(n h) s(n) f(n h) h^{d} .
$$

with error

$$
S-I=h^{d+m}\left(c_{0} f(0)+C_{2} h^{2}+\cdots+C_{2 \ell} h^{2 \ell}+O\left(h^{2 \ell+2}\right)\right),
$$

where $c_{0}$ is as in (3.7) and $C_{2}$ etc. depend on $g$. If $K$ is odd and $s$ is even, we have a similar expansion but with $h^{d+m}$ replaced by $h^{d+m+1}$, and the leading error is proportional to $\nabla f(0)$. 
Proof. The error from $(1-\zeta) f$ is easily seen to be high order. For $\zeta f$ we use a second order Taylor expansion for $f$, multiplied by $\zeta$,

$$
\zeta(x) f(x)=f(0) \zeta(x)+(\nabla f(0) \cdot x) \zeta(x)+\tilde{f}(x),
$$

where $\tilde{f}$ is a remainder of compact support. The constant term in $f$ leads to an integral whose quadrature error is the first term above plus a higher order part, according to the preceding lemma. The linear term in $f$ leads to an integrand which is odd in $x$, provided $K$ and $s$ are even; thus the integral for this term is zero, and the same is true for the sum, since the grid respects the symmetry.

Next we estimate the quadrature error coming from $\tilde{f}(x)$. The remainder $\tilde{f}$ consists of a sum of terms, each of the form $\gamma(x) b(x)$, where $b$ is bilinear and $\gamma$ is smooth. The integrand arising from such a term is

$$
F(x ; h)=K(x) s(x / h) b(x) \gamma(x)=h^{m+2} g(x / h) \gamma(x),
$$

where $g$ is the smooth function $K s b=K_{1} b$. In order to apply Lemma 3.1, we estimate $x$-derivatives of $F$. Now from the assumption (3.4) on $s$ and the homogeneity of $K$ we have $\left|D_{x}^{k} g(x)\right| \leq|x|^{m+2-k}$, so that

$$
\left.\mid D_{x}^{k} F(x ; h)\right)\left.|\leq C| x\right|^{m+2-k}, \quad|x| \geq h, \quad k \geq 0 .
$$

On the other hand, for $|x| \leq h$, we can use the smoothness of $K_{1}$ to estimate

$$
\left|D_{x}^{k} F(x ; h)\right| \leq C h^{m+2-k}, \quad|x| \leq h, \quad k \geq 0
$$

Combining these two pointwise estimates and integrating, we get

$$
\left|D_{x}^{k} F\right|_{L^{1}} \leq C h^{m+2+d-k}
$$

Lemma 3.1 now implies that the quadrature error is $O\left(h^{m+2+d}\right)$, and we have proved (3.16) with $\ell=0$.

The expansion can be carried further in the same way, as allowed by the smoothness, with zero contribution from the odd order terms in the Taylor series for $f$ by symmetry. If $K$ is odd rather than even, the sum and integral from the even terms in the Taylor series drop out, beginning with the constant $f(0)$.

Lemma 3.4. With $K$ and $K_{h}$ as in Lemmas 3.2 and [3.3, the constant $c_{0}$ in (3.7), (3.16) is given by

$$
c_{0}=(2 \pi)^{d / 2} \sum_{n \neq 0} \hat{K}_{1}(2 \pi n)
$$

Proof. The hypothesis on $K_{h}$ implies that $D^{j} K_{1}$ is in $L^{1}\left(\mathbf{R}^{d}\right)$ for $j$ large; consequently $\hat{K}_{1}(k)$ and its derivatives decrease rapidly for $k \neq 0$. We use Lemma 3.3 with a convenient choice of $f$. Suppose $f(0)=1$. Then, from (3.16) and (3.3),

$$
c_{0}=\lim _{h \rightarrow 0} h^{-(m+d)}(S-I)=\lim _{h \rightarrow 0}\left(\sum K_{1}(n) f(n h)-\int K_{1}(y) f(y h) d y\right) .
$$

We choose $f$ so that $\hat{f}(k)$ is smooth everywhere and zero outside $|k| \leq 1$. Now let $F_{h}(x)=K_{1}(x) f(x h)$. Then $F_{h}$ is smooth and rapidly decreasing, and we can apply the Poisson summation formula (with $k=0, h=1$ in (3.1) ) to obtain

$$
c_{0}=\lim _{h \rightarrow 0}(2 \pi)^{d / 2} \sum_{n \neq 0} \hat{F}_{h}(2 \pi n) .
$$


We need to justify passage to the limit to obtain (3.22). Since $F_{h}$ is a product, $\hat{F}_{h}$ is the convolution of $\hat{K}_{1}$ with $(2 \pi)^{-d / 2} h^{-d} \hat{f}(k / h)$, an approximate delta function. We can write $\hat{F}_{h}(k)-\hat{K}_{1}(k)$ as an integral and show by a standard argument, using the compact support of $\hat{f}$ and the decay of $D \hat{K}_{1}$, that $\left|\hat{F}_{h}(k)-\hat{K}_{1}(k)\right| \leq C h|k|^{-d-1}$ for $k \geq 1$. Thus the sum in (3.24) tends to the sum with $\hat{K}_{1}$ in place of $\hat{F}_{h}$.

We now apply the above lemmas to determine quadrature errors for single or double layer potentials on a doubly periodic surface, using the regularized Green's function of (2.1). We first deal with the neighborhood of the singularity. For convenience we place the singularity at zero in both $\alpha$ and $x$ coordinates. Recall that $G_{h}(x)$ is a smooth function of $x$ the form $r^{-1} s(r / h), r=|x|$, and $G_{h}(x)=$ $h^{-1} G_{1}(x / h)$.

Lemma 3.5. Let $\alpha \rightarrow x$ be a smooth mapping from a neighborhood $U$ of 0 in $\mathbf{R}^{2}$ into $\mathbf{R}^{3}$, with $0 \rightarrow 0$. Let $J$ be the Jacobian matrix $(\partial x / \partial \alpha)(0)$, so that $x=$ $J \alpha+O\left(\alpha^{2}\right)$. Assume that $J$ has full rank and $U$ is small enough so that

$$
|J \alpha| / 2 \leq|x| \leq 2|J \alpha|, \quad \alpha \in U .
$$

Let $G_{h}$ be a regularized Green's function obeying (2.1)-(2.3), and let $f(\alpha)$ be a smooth function with support in $U$. Then as $h \rightarrow 0$

$$
\sum_{n \in \mathbf{Z}^{2}} G_{h}(x(n h)) f(n h) h^{2}-\int_{U} G_{h}(x(\alpha)) f(\alpha) d \alpha=c_{0} f(0) h+O\left(h^{3}\right),
$$

where

$$
c_{0}=2 \pi \sum_{n \neq 0}\left(G_{1} \circ J\right)^{\wedge}(2 \pi n),
$$

or, with $\Gamma$ as in (2.14), $g_{\mu \nu}=\left(J^{*} J\right)_{\mu \nu}$, and $g^{\mu \nu}$ the inverse,

$$
c_{0}=2 \pi\left(\operatorname{det} g_{\mu \nu}\right)^{-1 / 2} \sum_{n \neq 0} \Gamma\left(2 \pi \sqrt{g^{\mu \nu} n_{\mu} n_{\nu}}\right) .
$$

Proof. Since $G_{1}$ is a smooth function of $x$, it is a smooth function of $|x|^{2}$, i.e., we can write $G_{1}(x)=g\left(|x|^{2}\right)$ for a smooth function $g$ of one variable, and therefore $G_{h}(x)=$ $h^{-1} g\left(\rho^{2}\right)$, where $\rho=|x| / h$. For later use we note that the decay assumption (2.2) for $s$ implies that

$$
\left|D^{k} g(\sigma)\right| \leq C|\sigma|^{-(k+1 / 2)}, \quad|\sigma| \geq 1 .
$$

We want to relate the composition $G_{h}(x(\alpha))$ to the simpler case where $x(\alpha)$ is replaced by $J \alpha$, a case to which the earlier lemmas apply. To this end we use a Taylor expansion for $g$ as a function of $\rho^{2}$ about $\rho_{1}^{2}$, and then set $\rho=|x(\alpha)| / h$ and $\rho_{1}=|J \alpha| / h$ :

$$
g\left(\rho^{2}\right)=g\left(\rho_{1}^{2}\right)+g^{\prime}\left(\rho_{1}^{2}\right)\left(\rho^{2}-\rho_{1}^{2}\right)+\tilde{g}\left(\rho_{1}, \rho\right)\left(\rho^{2}-\rho_{1}^{2}\right)^{2},
$$

with some smooth $\tilde{g}$. Further, we can write $x(\alpha)$ in a Taylor expansion about $\alpha=0$, beginning with $J \alpha$, to obtain

$$
\rho^{2}-\rho_{1}^{2}=h^{-2}\left(p_{3}(\alpha)+y(\alpha)\right),
$$

where $p_{3}$ is trilinear in $\alpha$ and the remainder $y$ has degree 4 or higher. Combining these, we have an expansion of $G_{h}$ in terms with successively higher homogeneity 
in $\alpha$ and $h$ together; we write $G_{h}=G_{h}^{(-1)}+G_{h}^{(0)}+G_{h}^{(1)}$, as a function of $\alpha$, where

$$
\begin{gathered}
G_{h}^{(-1)}=h^{-1} g\left(\rho_{1}^{2}\right)=G_{h}(J \alpha)=-(4 \pi|J \alpha|)^{-1} s(|J \alpha| / h), \\
G_{h}^{(0)}=h^{-3} g^{\prime}\left(\rho_{1}^{2}\right) p_{3}(\alpha),
\end{gathered}
$$

with nonhomogeneous remainder

$$
G_{h}^{(1)}=h^{-3} g^{\prime}\left(\rho_{1}^{2}\right) y+h^{-1} \tilde{g}\left(\rho_{1}, \rho\right)\left(\rho^{2}-\rho_{1}^{2}\right)^{2} .
$$

We multiply the expanded integrand by a cut-off function $\zeta(\alpha)$ which is even and equals 1 on the support of $f$, and assess the quadrature errors from the various terms. For the leading term $G_{h}^{(-1)}$, we have the integrand $G_{h}(J \alpha) f(\alpha) \zeta(\alpha)$. Lemma 3.3 applies with $m=-1$ and $x, K_{h}$ replaced by $\alpha, G_{h} \circ J$. Recalling (2.17), we obtain the first order error term as stated. For the next term we note that $p_{3}$ is odd in $\alpha$ and $\rho_{1}^{2}$ is even, so that $G_{h}^{(0)}(\alpha)$ is odd, and the contribution from $f(0)$ drops out. Using Lemma 3.3 we see that the error from $G_{h}^{(0)}(\alpha) f(\alpha) \zeta(\alpha)$ is $O\left(h^{3}\right)$.

Finally we verify that the quadrature error resulting from $G_{h}^{(1)}$ is $O\left(h^{3}\right)$, considering the two terms separately. The remainder $y$ in (3.31) is a sum of terms of the form $\tilde{y}(\alpha) p_{4}(\alpha)$, where $p_{4}$ is homogeneous of degree 4 and $\tilde{y}$ is smooth. Thus by Lemma 3.3 the integrand $h^{-3} g^{\prime}\left(\rho_{1}^{2}\right) \tilde{y}(\alpha) p_{4}(\alpha)$ has quadrature error $O\left(h^{-3+4+2}\right)=O\left(h^{3}\right)$. For the last term we need to estimate directly. The remainder $\tilde{g}$ in $g$ is

$$
\tilde{g}\left(\rho_{1}, \rho\right)=\int_{0}^{1} t g^{\prime \prime}\left(t \rho_{1}^{2}+(1-t) \rho^{2}\right) d t .
$$

Using (3.29) and (3.25), we can conclude that

$$
\left|D_{\alpha}^{k} \tilde{g}\left(\rho_{1}, \rho\right)\right| \leq C h^{5}|\alpha|^{-(5+k)}, \quad|\alpha| \geq c_{1} h,
$$

with $c_{1}$ a constant large enough so that $|\alpha| \geq c_{1} h$ implies $|x| \geq h$. We can write $\rho^{2}-\rho_{1}^{2}$ as a sum of terms of the form $h^{-2} q_{3}(\alpha) r(\alpha)$, where $q_{3}$ is trilinear. Thus the last term in (3.34) leads to the integrand $F(\alpha)=h^{-5} \tilde{g}\left(\rho_{1}, \rho\right) q_{3}(\alpha)^{2} r(\alpha)^{2} \zeta(\alpha)$. We estimate the quadrature error using Lemma 3.1. Using (3.36), we find that

$$
\left|D^{k} F(\alpha)\right| \leq C|\alpha|^{1-k}, \quad|\alpha| \geq c_{1} h .
$$

On the other hand, for $|\alpha| \leq c_{1} h$, we have $\left|D_{\alpha}^{k} \tilde{g}\right|=O\left(h^{-k}\right)$, and consequently

$$
\left|D^{k} F(\alpha)\right| \leq C h^{1-k}, \quad|\alpha| \leq c_{1} h .
$$

Combining these two pointwise estimates, we have $\left|D^{k} F\right|_{L^{1}} \leq C h^{3-k}$, and by Lemma 3.1 the quadrature error is $O\left(h^{3}\right)$.

The next lemma concerns the double layer potential near the singularity. We recall that the gradient of the regularized Green's function, $\nabla G_{h}$, has the form $r^{-3} x \tilde{s}(|x| / h)$. We assume it is multiplied by a vector-valued function $F(\alpha)$ which is normal at the singular point, so that the product is integrable.

Lemma 3.6. Assume the mapping $\alpha \rightarrow x$, the set $U$, and the function $G_{h}$ are as in Lemma 3.5 Let $F(\alpha)$ be a smooth vector-valued function with support in $U$ such that $F(0)$ is parallel to $n_{0}$, the unit normal to the surface $x(\alpha)$ at $x(0)=0$. Then

$$
\sum_{n \in \mathbf{Z}^{2}} \nabla G_{h}(x(n h)) \cdot F(n h) h^{2}-\int_{U} \nabla G_{h}(x(\alpha)) \cdot F(\alpha) d \alpha=C_{0} h+O\left(h^{3}\right),
$$


for some $C_{0}$ depending on $F$. In particular, $C_{0}=0$ and the error is $O\left(h^{3}\right)$ under the stronger assumption

$$
F(\alpha) \cdot n_{0}=O(\alpha) \quad \text { and } \quad F^{T}(\alpha)=O\left(\alpha^{2}\right) \quad \text { as } \quad \alpha \rightarrow 0,
$$

where $F^{T}(\alpha)$ is the projection of $F(\alpha)$ orthogonal to $n_{0}$.

Proof. We use an argument like the proof of Lemma 3.5. The function $\tilde{s}$ in $\nabla G_{h}$ (cf. (2.11)) has the form $\tilde{s}(\rho)=\rho^{3} g\left(\rho^{2}\right)$, where $g$ is a smooth function, so that

$$
\nabla G_{h}(x)=h^{-3} g\left(\rho^{2}\right) x, \quad \rho=|x| / h .
$$

Let $x(\alpha)=x_{1}(\alpha)+x_{2}(\alpha)+\ldots$ be the Taylor expansion for $x(\alpha)$ about $\alpha=0$, with $x_{1}=J \alpha$ linear, $x_{2}$ bilinear, etc. As in (3.30) we expand $g\left(\rho^{2}\right)$ about $\rho_{1}^{2}$, with $\rho_{1}=\left|x_{1}\right| / h$, and substitute for $\rho^{2}-\rho_{1}^{2}$ in terms of the $x$ 's. We collect terms according to degree in $\alpha, h$ together. In this way we write $\nabla G_{h}=K_{-2}+K_{-1}+K_{0}+K_{1}+\ldots$ as functions of $\alpha$. The first two are

$$
\begin{gathered}
K_{-2}=h^{-3} g\left(\rho_{1}^{2}\right) x_{1}, \\
K_{-1}=h^{-3} g\left(\rho_{1}^{2}\right) x_{2}+2 h^{-5} g^{\prime}\left(\rho_{1}^{2}\right)\left(x_{1} \cdot x_{2}\right) x_{1} .
\end{gathered}
$$

We consider first the quadrature of $K_{-2}(\alpha) \cdot F(\alpha)$. (As usual we multiply by a cut-off covering the support of $F$.) The term $K_{-2}(\alpha) \cdot F(0)$ is zero, since $x_{1}(\alpha)$ is tangent and $F(0)$ is normal by hypothesis. Then Lemma 3.3 implies that the quadrature error has the form $C_{0} h+O\left(h^{3}\right)$, with $C_{0}$ determined by the scalar product of $x_{1}(\alpha)$ with the linear term in the expansion of $F$. Under the hypothesis (3.40), this linear term is parallel to $n_{0}$, so that the $O(h)$ error vanishes. For $K_{-1}$, we again have an error of the form $C h+O\left(h^{3}\right)$, according to Lemma 3.3 The $O(h)$ term comes from the constant $F(0)$, and it vanishes under hypothesis (3.40). It can be seen that $K_{0}$ is odd in $\alpha$, so that by Lemma 3.3 it contributes an error $O\left(h^{3}\right)$. Similar considerations show that higher order terms beyond $K_{0}$ lead to errors $O\left(h^{3}\right)$ or smaller. To obtain terms up to $K_{0}$, we need to expand $g$ to the second order, $x$ to the third order, and $\rho^{2}-\rho_{1}^{2}$ to the fourth degree terms, all with remainders. Finally, we can estimate the quadrature errors due to the remainders, in a manner similar to Lemma 3.5, and show that they are $O\left(h^{3}\right)$. The remainder in $g\left(\rho^{2}\right)$ has the form $\tilde{g}\left(\rho, \rho_{1}\right)\left(\rho^{2}-\rho_{1}^{2}\right)^{3}$, where $\tilde{g}$ is an average of $g^{\prime \prime \prime}$. In this case $g\left(\rho^{2}\right)$ decays like $\rho^{-3}$, and the assumptions on the decay of $\tilde{s}$ imply that $\left|D_{\alpha}^{k} \tilde{g}\left(\rho, \rho_{1}\right)\right| \leq C|\alpha|^{-(9+k)}$ for $|\alpha| \geq c_{1} h$. Otherwise the treatment of this term proceeds as before.

Now that we have treated the quadrature of the single or double layer potential near the singularity, we can obtain similar results for the integral over the doubly periodic surface.

Theorem 3.7. Let $\alpha \rightarrow x$ be a smooth mapping from $\mathbf{R}^{2}$ to $\mathbf{R}^{3}$ such that $x(\alpha)-$ $(\alpha, 0)$ is doubly $2 \pi$-periodic and (1.13) holds. Let $f$ be a smooth periodic function of $\alpha$, and $G_{h}^{\pi}$ a regularized periodic Green's function as in (2.1)-(2.3), (2.26). Let $\alpha_{j}=j h$ for $j \in \mathbf{Z}^{2}$, and $x_{j}=x\left(\alpha_{j}\right)$. Then, for each $j \in \mathbf{Z}^{2}$,

$$
\sum_{\ell \in I} G_{h}^{\pi}\left(x_{j}-x_{\ell}\right) f\left(\alpha_{\ell}\right) h^{2}-\int G_{h}^{\pi}\left(x_{j}-x\left(\alpha^{\prime}\right)\right) f\left(\alpha^{\prime}\right) d \alpha^{\prime}=\gamma_{j} f\left(\alpha_{j}\right) h+O\left(h^{3}\right),
$$


where $I$ is the index set (1.10) and the integral is over one period square. Here

$$
\gamma_{j}=2 \pi\left(\operatorname{det} g^{\mu \nu}\left(\alpha_{j}\right)\right)^{1 / 2} \sum_{0 \neq n \in \mathbf{Z}^{2}} \Gamma\left(2 \pi \sqrt{g^{\mu \nu}\left(\alpha_{j}\right) n_{\mu} n_{\nu}}\right)
$$

with $\Gamma$ as in (2.14) and $g^{\mu \nu}$ the inverse metric tensor.

Similarly, let $\tilde{f}\left(\alpha, \alpha^{\prime}\right)$ be smooth and periodic in $\alpha, \alpha^{\prime}$, and let $n\left(\alpha^{\prime}\right)$ be the unit normal vector at $x\left(\alpha^{\prime}\right)$. Then the quadrature error for the integral

$$
\int \nabla G_{h}^{\pi}\left(x_{j}-x\left(\alpha^{\prime}\right)\right) \cdot n\left(\alpha^{\prime}\right) \tilde{f}\left(\alpha_{j}, \alpha^{\prime}\right) d \alpha^{\prime} .
$$

is $O(h)$. If $\tilde{f}\left(\alpha, \alpha^{\prime}\right)=0$ for $\alpha=\alpha^{\prime}$, the error improves to $O\left(h^{3}\right)$.

Proof. We need to reduce the general case to the local estimates of Lemmas 3.5 and 3.6. As in the proof of Theorem 2.2, we can introduce a cut-off function $\zeta(\alpha)$ with support near zero and write $G_{h}^{\pi}\left(x_{j}-x\left(\alpha^{\prime}\right)\right)$ as a sum $G_{h 0}^{\pi}\left(\alpha^{\prime}\right)+G_{h \infty}^{\pi}\left(\alpha^{\prime}\right)$, depending on $j$, where the singularity is localized in $G_{h 0}^{\pi}\left(\alpha^{\prime}\right)$, and $G_{h \infty}^{\pi}\left(\alpha^{\prime}\right)$ is smooth, with derivatives bounded uniformly in $h$. We take the domain of integration to be the period square $S_{j}$ centered at $\alpha_{j}$. We can now split the single layer integral into two parts. The integral with $G_{h \infty}^{\pi}\left(\alpha^{\prime}\right)$ has smooth periodic integrand, and its quadrature is high order accurate. The remaining part of the quadrature error comes from the integral with $G_{h 0}^{\pi}$. As in the proof of Theorem $2.2 G_{0}^{\pi}\left(\alpha^{\prime}\right)=$ $G_{h}\left(x_{j}-x\left(\alpha^{\prime}\right)\right) \zeta\left(\alpha_{j}-\alpha^{\prime}\right)$ for $\alpha^{\prime} \in S_{j}$. Thus the single-layer integral is reduced to the case of Lemma 3.5, we need to choose the support of $\zeta$ so that (3.25) holds, depending on the mapping $x(\alpha)$. For the double layer integral we use a similar argument to reduce to the case of Lemma 3.6. the hypothesis (3.40) holds when $\tilde{f}=0$ for $\alpha=\alpha^{\prime}$.

We can apply this theorem to the discretization error for the four sums representing integrals in the discrete integral equation (1.25)-(1.27). This error results not only from the quadrature but also from the replacement of the derivative by the discrete operator $D_{h}$, and we begin with the latter. If $f$ is a smooth, doubly periodic function of $\alpha$, we can regard the values of $D_{h} f$ on the grid as restrictions of a smooth function $D_{h} f$, defined for all $\alpha$ using the Fourier series for $f$. Then $D_{h} f=D f+h^{3} r$, where $r$ is a smooth function of $\alpha$, depending on $h$, with a high norm bounded uniformly in $h$. Thus $X_{k}^{(h)}$, the tangent vector computed using $D_{h}$, differs from the exact tangent vector $X_{k}$ by a smooth function of order $h^{3}$, and similarly for $N, n, X_{k}^{*}$. In the same way, $-D_{h}^{*}$ differs from $D$ to $O\left(h^{3}\right)$.

We first compare the integral

$$
I \equiv \int \nabla^{T} G_{h}^{\pi}\left(x_{j}-x\left(\alpha^{\prime}\right)\right) \cdot n\left(\alpha_{j}\right) \phi_{n}\left(\alpha^{\prime}\right)\left|N\left(\alpha^{\prime}\right)\right| d \alpha^{\prime}
$$

as in (1.26) with the sum

$$
S \equiv \sum_{\ell} \nabla_{h}^{T} G_{h}^{\pi}\left(x_{j}-x_{\ell}\right) \cdot n_{j}^{(h)}\left(\phi_{n}\right)_{\ell}\left|N_{\ell}^{(h)}\right| h^{2}
$$

and derive a correction so that the remaining error is $O\left(h^{3}\right)$. (Upper $(h)$ indicates the use of $D_{h}$. We have $G_{h}$ in the integral since we have already treated the error in regularizing $G$ in $₫ 2$ ) We start by writing out $\nabla^{T}$ in the integral and integrating 
by parts to obtain (with sum over $k=1,2$ and $D_{k}$ the derivative in $\alpha_{k}^{\prime}$ )

$$
I=\int G_{h}^{\pi}\left(x_{j}-x\left(\alpha^{\prime}\right)\right) D_{k}\left\{X_{k}^{*}\left(\alpha^{\prime}\right) \cdot n\left(\alpha_{j}\right) \phi_{n}\left(\alpha^{\prime}\right)\left|N\left(\alpha^{\prime}\right)\right|\right\} d \alpha^{\prime} .
$$

Using the remarks above, we can replace $X_{k}^{*}\left(\alpha^{\prime}\right)$ by $X_{k}^{*(h)}\left(\alpha^{\prime}\right)$ etc., with pointwise error $O\left(h^{3}\right)$. We also replace $D_{k}$ outside with $-D_{h, k}^{*}$. Since $G_{h}^{\pi}$ is integrable, the error in the integral is $O\left(h^{3}\right)$. We now have

$$
I=-\int G_{h}^{\pi}\left(x_{j}-x\left(\alpha^{\prime}\right)\right) D_{h, k}^{*}\left\{X_{k}^{*(h)}\left(\alpha^{\prime}\right) \cdot n_{j}^{(h)} \phi_{n}\left(\alpha^{\prime}\right)\left|N^{(h)}\left(\alpha^{\prime}\right)\right|\right\} d \alpha^{\prime}+O\left(h^{3}\right) .
$$

Similarly we write out $\nabla_{h}^{T}$ in the sum and use summation by parts, obtaining

$$
S=-\sum_{\ell} G_{h}^{\pi}\left(x_{j}-x_{\ell}\right) D_{h, k}^{*}\left\{X_{k \ell}^{*(h)} \cdot n_{j}^{(h)}\left(\phi_{n}\right)_{\ell}\left|N_{\ell}^{(h)}\right|\right\} h^{2},
$$

where $D_{h, k}^{*}$ acts on the $\ell$ variable. With the integral and sum in this form, the above theorem applies directly; up to $O\left(h^{3}\right)$, the error is the $O(h)$ term with coefficient $f\left(\alpha_{j}\right)$ given by

$$
r_{j}=-\left.D_{h, k}^{*}\left\{X_{k \ell}^{*(h)} \cdot n_{j}^{(h)}\left(\phi_{n}\right)_{\ell}\left|N_{\ell}^{(h)}\right|\right\}\right|_{\ell=j} .
$$

To simplify this, we can replace $-D_{h}^{*}$ by $D$, use the fact that $X_{k j}^{*(h)} \cdot n_{j}^{(h)}=0$, and then replace $D$ with $-D_{h}^{*}$, committing $O\left(h^{3}\right)$ errors, to rewrite (3.52) as

$$
r_{j}=-\left(D_{h, k}^{*} X_{k}^{*(h)}\right)_{j} \cdot N_{j}^{(h)}\left(\phi_{n}\right)_{j} .
$$

In summary, the integral (3.47) and the sum (3.48) differ by

$$
S-I=\gamma_{j} r_{j} h+O\left(h^{3}\right) .
$$

It is this correction term that appears in 1.26), (1.29) except for the operator $P_{h}$. The smoothing by $\rho(k \sqrt{h})$ contributes an error of order $h \cdot h^{2}=h^{3}$, provided $\rho(\xi)-1=O\left(\xi^{4}\right)$ as in (1.33).

The first sum in (1.27) can be treated analogously. The correction simplifies since the last factor vanishes at $\ell=j$, and we obtain (1.30). We can use a similar argument to show that the two sums in (1.26), (1.27) with normal derivative of $G_{h}^{\pi}$ differ from the corresponding integrals by $O\left(h^{3}\right)$. After replacing $D$ with $D_{h}$ again, we have a sum minus an integral with kernel $\nabla G_{h}^{\pi} \cdot N_{\ell}^{(h)}$. We cannot apply the second part of Theorem 3.7 directly, since $N_{\ell}^{(h)}$ is not exactly normal at $x\left(\alpha_{\ell}\right)$. However, we can write it as the exact $N\left(\alpha_{\ell}\right)$ plus a smooth remainder of order $h^{3}$. The theorem applies to the main part, since the integrand has a factor which vanishes on the diagonal. For the remainder, an argument like Lemmas 3.5 and 3.6 shows that the quadrature for $\nabla G_{h}^{\pi}$ has $O(1)$ error, and this is multiplied by $O\left(h^{3}\right)$ in our remainder term. The total quadrature error is thus $O\left(h^{3}\right)$.

\section{Discrete PSEudodifferential operators}

In this section we define a class of discrete pseudodifferential operators and derive basic properties such as boundedness on $L_{h}^{2}$. We use a symbol class which allows us to compose operators by multiplying symbols, in some cases, with bounded remainder. We prove a discrete version of Gårding's inequality and related facts which will be applied to the operator $\Lambda$ in 6 . We interpret discrete integral operators in this 
context and express the symbol in terms of the Fourier transform. This connection will be used in $\$ 5$ For the usual theory of pseudodifferential operators, see e.g., [30]. For an extensive theory of discrete operators with high wavenumber cut-off, see [25].

We will work with operators acting on functions defined on the grid $I h$, with $I$ as in (1.10). The general form of a discrete pseudodifferential operator $A$ is

$$
A f(j h)=(2 \pi)^{-2} \sum_{k \in I} \sum_{\ell \in I} a(j h, \ell h, k ; h) e^{i k(j-\ell) h} f(\ell h) h^{2}
$$

or

$$
A f(x)=(2 \pi)^{-2} \sum_{k \in I} \sum_{y \in I h} a(x, y, k ; h) e^{i k(x-y)} f(y) h^{2}, \quad x \in I h .
$$

We generally assume $a(j h, \ell h, k ; h)$ is extended periodically to all integer $j, \ell, k$. We will often omit the $h$-dependence in writing $a$, although it will be important that our estimates have constants independent of $h$.

In the special case where $a$ is independent of the second variable $\ell h$ or $y$, we can rewrite the operator (4.1) as

$$
A f(j h)=\sum_{k} a(j h, k ; h) e^{i k j h} \ddot{f}(k),
$$

where $\ddot{f}$ is the discrete Fourier transform (1.11). This is the discrete analogue of the standard form of a pseudodifferential operator. On the other hand, if $a$ is independent of the first variable $j h$ or $x$, we have from (4.1)

$$
(A f){ }^{*}(k)=(2 \pi)^{-2} \sum_{\ell} a(\ell h, k ; h) e^{-i k \ell h} f(\ell h) h^{2} .
$$

As usual we refer to $a$ as the symbol of the operator $A$. The following lemma is a direct consequence of the definitions.

Lemma 4.1. Suppose $A$ is an operator of type (4.2) with symbol $a(j h, k)$ and $B$ is an operator of type (4.3) with symbol b(lh,k). Then the composition $A B$ is of type (4.1) with symbol $a(j h, k) b(\ell h, k)$. The adjoint of $A$ is an operator $A^{*}$ of type (4.3) with symbol $\bar{a}(\ell h, k)$.

Often we will want to assume that the symbol $a(j h, \ell h, k ; h)$ is smooth in $j h, \ell h$ with order $m$ in $k$, at least in the sense that

$$
\left|D_{+}^{s} a(j h, \ell h, k ; h)\right| \leq C(|k|+1)^{m}
$$

with $C$ independent of $j h, \ell h \in I h, k \in I$, as well as $h$. Here $s$ is a multi-index, arbitrary up to some large order, and $D_{+}$is a forward divided difference in $j h$ or $\ell h$. The next lemma gives the $L_{h}^{2}$ boundedness of an operator with such a symbol with order $m=0$.

Lemma 4.2. Assume (4.4) holds with $m=0$ and with s large. Then

(i) The discrete transform $\ddot{a}(\xi, \eta, k ; h)$ of a with respect to $(x, y)$ satisfies

$$
|\ddot{a}(\xi, \eta, k ; h)| \leq C\left(1+|\xi|^{2}+|\eta|^{2}\right)^{-\nu / 2}
$$

with $\nu$ large depending on $s$.

(ii) The operator A defined by (4.1) is bounded in norm on $L_{h}^{2}$ uniformly in $h$. 
Proof. For (i), we note that the five-point Laplacian of $a$ in $j h$ is bounded by hypothesis, and therefore its transform $-4 h^{-2}\left(\sin ^{2}\left(\xi_{1} h / 2\right)+\sin ^{2}\left(\xi_{2} h / 2\right)\right) \ddot{a}(\xi, \eta, k)$ is also. This is at least $(2 / \pi)^{2}|\xi|^{2}|\ddot{a}|$ in magnitude. Similarly, using powers of the discrete Laplacian in $j h$ or $\ell h$, we obtain the estimate (4.5).

Part (ii) can be proved by adapting a standard argument, e.g., [30], Prop. II.6.1. With $x, y \in I h$ we can write

$$
\begin{gathered}
a(x, y, k)=\sum_{\xi, \eta \in I} \ddot{a}(\xi, \eta, k) e^{i \xi x} e^{i \eta y}, \quad A=\sum_{\xi, \eta \in I} A_{\xi \eta}, \\
A_{\xi \eta} f(x)=(2 \pi)^{-2} \sum_{k} \sum_{\ell} \ddot{a}(\xi, \eta, k) e^{i \xi x} e^{i \eta \ell h} e^{i k(x-\ell h)} f(\ell h) h^{2} .
\end{gathered}
$$

Now

$$
A_{\xi \eta} f(x)=\sum_{k \in I} e^{i(k+\xi) x} \ddot{a}(\xi, \eta, k) \ddot{f}(k-\eta) .
$$

Each factor is periodic in $k$, and we can shift $k$ to rewrite this as

$$
A_{\xi \eta} f(x)=\sum_{k \in I} e^{i(k+\xi+\eta) x} \ddot{a}(\xi, \eta, k+\eta) \ddot{f}(k) .
$$

Thus $A_{\xi \eta} f(x)$ is $e^{i(\xi+\eta) x}$ times a function whose transform is $\ddot{a}(\xi, \eta, k+\eta) \ddot{f}(k)$. It follows from (i) that $\left|A_{\xi \eta} f\right|_{L^{2}} \leq C\left(\left(1+|\xi|^{2}+|\eta|^{2}\right)^{-\nu / 2}|f|_{L^{2}}\right.$. Then $|A f|_{L^{2}}$ is bounded by the sum of such estimates; we obtain the result provided $\nu$ is large enough so that the sum over $\xi, \eta$ has an upper bound independent of $h$.

For further results we need an assumption that the undivided difference in $k$, written as $\Delta_{+(k)}$, reduces the order of the symbol by 1 . For this reason we define a symbol class somewhat like that of the continuous case. We will say that $a(x, y, k ; h)$ is a symbol of class $S^{m}$, for real $m$, if $a(x, y, k ; h)$ is defined for all $x, y \in R^{2}$ and integer $k$, is periodic in each variable, and satisfies the estimate

$$
\left|\Delta_{+(k)}^{n} D^{s} a(x, k ; h)\right| \leq C(1+|k|)^{m-|n|}, \quad k \in I, \quad|n|=0 \text { or } 1,
$$

where $n$ and $s$ are multi-indices and $D^{s}$ is an $(x, y)$-derivative, with $s$ up to large degree. Note that (4.10) is required to hold even at the border points of $I$. We are primarily interested in the special case of a symbol for an operator of type (4.2) or (4.3). The following lemma shows that the composition of two operators of type (4.2) and (4.3) with total order $\leq 1$ can be written as an operator of type (4.2) plus a bounded remainder.

Lemma 4.3. Let $a(x, k ; h), b(y, k ; h)$ be symbols of class $S^{m_{1}}, S^{m_{2}}, m_{1}+m_{2} \leq 1$. Let $A, B$ be the operators of type (4.2), (4.3) corresponding to $a(j h, k ; h), b(\ell h, k ; h)$. Also let $M$ be the operator of type (4.2) with symbol $a(j h, k ; h) b(j h, k ; h)$. Then $M-A B$ is bounded as an operator on $L_{h}^{2}$, uniformly in $h$.

Proof. The operator $M-A B$ has type (4.1) with symbol $a(x, k)(b(x, k)-b(y, k))$. We want to perform a summation by parts using

$$
\Delta_{+\nu} e^{i k(x-y)}=\left(e^{i\left(x_{\nu}-y_{\nu}\right)}-1\right) e^{i k(x-y)},
$$

where $\Delta_{+\nu}$ is the forward difference in $k_{\nu}$. We can write

$$
b(x, k)-b(y, k)=\sum_{\nu=1,2} q_{\nu}(x, y, k)\left(e^{i\left(x_{\nu}-y_{\nu}\right)}-1\right)
$$


with

$$
q_{1}=\frac{b\left(x_{1}, x_{2}, k\right)-b\left(y_{1}, x_{2}, k\right)}{e^{i\left(x_{1}-y_{1}\right)}-1}, \quad q_{2}=\frac{b\left(y_{1}, x_{2}, k\right)-b\left(y_{1}, y_{2}, k\right)}{e^{i\left(x_{2}-y_{2}\right)}-1} .
$$

The denominator in $q_{\nu}$ vanishes only where $x_{\nu}=y_{\nu}$ modulo $2 \pi$, and $q_{\nu}$ can be extended there so that $q_{\nu}$ is smooth and periodic in $x, y$. It can be checked that $q_{\nu}$ satisfies the same estimates as $b$. We now have

$$
(M-A B) f(x)=(2 \pi)^{-2} \sum_{\nu, k, y} a(x, k) q_{\nu}(x, y, k) \Delta_{+\nu} e^{i k(x-y)} f(y) h^{2},
$$

or, with a summation by parts,

$$
(M-A B) f(x)=-(2 \pi)^{-2} \sum_{\nu, k, y} \Delta_{-\nu}\left(a(x, k) q_{\nu}(x, y, k)\right) e^{i k(x-y)} f(y) h^{2},
$$

using the periodicity in $k$. The estimates for $a, b, q_{\nu}$ imply that $\Delta_{-\nu} D_{x, y}^{s}\left(a q_{\nu}\right)$ is uniformly bounded for large $s$, independent of $k, h$, and the same holds if the $(x, y)$ derivatives are replaced by differences. Finally we can conclude from Lemma 4.2 that the operator $M-A B$ is $L_{h}^{2}$-bounded, independent of $h$.

The next three results use this lemma. It is convenient to use an operator $E$ on grid functions representing an absolute first derivative, defined as

$$
(E f)^{*}(k)=\left(1+|k|^{2}\right)^{1 / 2} \ddot{f}(k), \quad k \in I .
$$

More generally, we define $E^{r}$ as multiplication by $\left(1+|k|^{2}\right)^{r / 2}$ in the transform for real $r$. It is important to us that this factor is itself in class $S^{r}$, i.e., first differences in $k$ of the periodic extension reduce the order in $k$.

Lemma 4.4. Let $A$ be an operator of type (4.2) with symbol $a(x, k ; h)$ in the class $S^{1}$. Then there are operators $B_{1}, B_{2}, B_{3}$, bounded on $L_{h}^{2}$ uniformly in $h$, so that

$$
A=B_{1} E, \quad A=E B_{2}, \quad A=E^{1 / 2} B_{3} E^{1 / 2} .
$$

Proof. Let $b(x, k ; h)=a(x, k ; h) \eta(k)^{-1}$ for $k \in I$, where $\eta(k)=\left(1+|k|^{2}\right)^{1 / 2}$, and extend periodically in $k$. Then $b$ is a symbol of class $S^{0}$. Let $B_{1}$ be the operator of type (4.2) with symbol $b(x, k ; h)$. Then $B_{1}$ is bounded uniformly in $h$, according to Lemma 4.2, and $A=B_{1} E$. This gives the first equation. For the second, we start with $B_{0} \equiv B_{1}^{*}$, a bounded operator of type (4.3) with symbol $b(y, k ; h)$. We apply Lemma 4.3 with $E, B_{0}$ in place of $A, B$, and $m_{1}=1, m_{2}=0$. We conclude that $E B_{0}$ differs by a bounded operator from the type (4.2) operator with symbol $\eta(k) b(x, k ; h)$, namely $A$. Thus we have $A=E B_{0}+B_{2}$ for some $B_{2}$, or $A=E\left(B_{0}+E^{-1} B_{2}\right)$, which is the second equation except for notation.

The third equation is proved similarly. Let $R$ be the operator of type (4.2) with symbol $r(x, k ; h)=a(x, k ; h) \eta(k)^{-1 / 2}$ for $k \in I$, so that $r$ is of class $S^{1 / 2}$, $A=R E^{1 / 2}$, and $r=\eta(k)^{1 / 2} b$. We apply Lemma 4.3 to the operators $E^{1 / 2}, B_{0}$, with $B_{0}$ as above, and conclude that $R=E^{1 / 2} B_{0}+B_{4}$ for some bounded $B_{4}$. Then $A=\left(E^{1 / 2} B_{0}+B_{4}\right) E^{1 / 2}=E^{1 / 2}\left(B_{0}+E^{-1 / 2} B_{4}\right) E^{1 / 2}$.

Next we derive a result like Gårding's inequality, relating the positivity of a symbol of class $S^{1}$ to the positivity of the operator. The resulting estimate is crucial for the numerical stability estimates of 96 . 
Lemma 4.5. Let $a(x, k ; h)$ be a symbol of class $S^{1}$. Assume that $a(x, k ; h) \geq c_{0}|k|$ for $k \in I$ and all $x$, with constant $c_{0}>0$. Let $A$ be the operator of type (4.2) with symbol $a(x, k ; h)$. Then $A^{*}-A$ is $L_{h}^{2}$-bounded, uniformly in $h$, and for all grid functions $f$ and some $C \geq 0$,

$$
\operatorname{Re}(A f, f)_{L_{h}^{2}} \geq \frac{c_{0}}{2} \sum_{k \in I}|k||\ddot{f}(k)|^{2}-C|f|_{L_{h}^{2}}^{2} .
$$

Proof. We mimic the standard proof (e.g., 30], Lemma II.6.2), using Lemma 4.3 We may assume that $a(x, k ; h) \geq c_{0} \eta(k)$ for $k \in I$, where $\eta(k)=\left(1+|k|^{2}\right)^{1 / 2}$, by adding a constant to $a$ without affecting the conclusion. We set $b(x, k ; h)=$ $a(x, k ; h) / \eta(k)$ for $k \in I$ and extend periodically in $k$. Then $b$ is a symbol of class $S^{0}$. Let $a_{1}(x, k ; h)=a(x, k ; h)-\left(c_{0} / 2\right) \eta(k)$ for $k \in I$, and let $A=A_{0}+A_{1}$, where $A_{0}$ has symbol $\left(c_{0} / 2\right) \eta(k)$ and $A_{1}$ has symbol $a_{1}(x, k ; h), k \in I$. Then $a_{1}(x, k ; h)=\eta(k)\left(b(x, k ; h)-c_{0} / 2\right)$ for $k \in I$; note the last factor is at least $c_{0} / 2$. We want to approximate $A_{1}$ by an operator of the form $R R^{*}$, which is necessarily positive. Let $r(x, k)=\eta(k)^{1 / 2}\left[b(x, k ; h)-c_{0} / 2\right]^{1 / 2}$. We check that $r$ is a symbol of class $S^{1 / 2}$ : The bound for $D_{x}^{s} r$ follows from the one for $D_{x}^{s} b$. As for the $k$-difference, the estimate for $\Delta_{+(k)} D_{x}^{s} b$ implies that $\left|\Delta_{+(k)} D_{x}^{s}\left[b(x, k ; h)-c_{0} / 2\right]^{1 / 2}\right| \leq C \eta(k)^{-1}$ and therefore $\left|\Delta_{+(k)} D_{x}^{s} r(x, k ; h)\right| \leq C \eta(k)^{-1 / 2}$. Thus Lemma 4.3 applies to the operator $R$ with symbol $r(x, k ; h)$ and the adjoint $R^{*}$ with symbol $r(y, k ; h)$. We conclude that $A_{1}=R R^{*}+B$ with $B$ bounded. Since $R R^{*}$ and $A_{0}$ are self-adjoint, $A^{*}-A=B^{*}-B$, and the first statement is proved. Further, we have

$$
(A f, f)_{L_{h}^{2}}=\left(A_{0} f, f\right)_{L_{h}^{2}}+\left|R^{*} f\right|_{L_{h}^{2}}^{2}+(B f, f)_{L_{h}^{2}}
$$

and the second statement follows.

The next lemma expresses the absolute derivative $E$ in terms of the operator $A$ of Lemma 4.5, this is needed in $\$ 6$.

Lemma 4.6. Let $A$ be as in Lemma 4.5 Then for each $h$ there are operators $B_{4}, B_{5}$, bounded on $L_{h}^{2}$ uniformly in $h$, so that $E=B_{4} A+B_{5}$.

Proof. Let $Q$ be the operator of type (4.3) with symbol $b^{-1}$, where $b=a / \eta$ for $k \in I$. Lemma 4.3 applies with $A, Q$ in place of $A, B$, and $m_{1}=1, m_{2}=0$. We conclude that $A Q$ differs by a bounded operator from the operator of type (4.2) with symbol $a b^{-1}=\eta$; That is, $A Q=E+B_{1}$ for some bounded $B_{1}$. Now $Q$ is bounded, according to Lemma 4.2 Taking adjoints, we get $Q^{*} A^{*}=E+B_{1}^{*}$. Then, since $A^{*}-A \equiv B_{3}$ is a bounded operator, we have $B_{4} A=E+B_{1}^{*}-B_{4} B_{3}$, with $B_{4}=Q^{*}$, or $E=B_{4} A+B_{5}$.

In dealing with discrete potentials, we will apply the above to discrete integral operators of the form

$$
A f(j h)=\sum_{\ell \in I} K^{\pi}(j h, j h-\ell h) f(\ell h) h^{2}
$$

where $K^{\pi}(x, z)$ is periodic in both $x$ and $z$, and $K^{\pi}$ has the form

$$
K^{\pi}(x, z)=\sum_{n \in Z^{2}} K(x, z+2 \pi n)
$$

for a function $K(x, z)$ which is smooth in $x$ and $z$, periodic in $x$, and rapidly decreasing in $z$. For each $j$, we can regard the sum (4.20) as a discrete convolution 
of $K^{\pi}(j h, \cdot)$ with $f$, evaluated at $j h$. Then $A f$ is the inverse discrete transform of a product,

$$
A f(j h)=(2 \pi)^{2} \sum_{k \in I} \ddot{K}^{\pi}(j h, k) e^{i k j h} \ddot{f}(k),
$$

an operator of type (4.2). Furthermore, since $K^{\pi}$ has the form (4.21), its transform as a discrete periodic function can be written as a discrete transform of $K$ itself,

$$
\ddot{K}^{\pi}(j h, k)=(2 \pi)^{-2} \sum_{m \in Z^{2}} K(j h, m h) e^{-i k m h} h^{2} .
$$

The latter can be related to the usual Fourier transform of $K$, as a function of $z$, using the Poisson summation formula (3.1):

$$
\ddot{K}^{\pi}(j h, k)=(2 \pi)^{-1} \sum_{n \in Z^{2}} \hat{K}(j h, k+2 \pi n / h) .
$$

This relation will allow us to study operators related to $G_{h}^{\pi}$ by estimating their transforms, and to derive an important positivity property using Lemma 4.5 .

\section{Estimates FOR INTEGRAL OPERATORS}

In this section we derive boundedness properties on $L_{h}^{2}$ of discrete integral operators with kernels related to the regularized Green's function. We expand $G_{h}$ near the singularity into terms where derivatives of $G_{h}$ are composed with the linearized coordinate mapping. The Fourier transforms of such terms are estimated. It is then shown, using the results of 4 , that various operators are bounded, with gain of derivatives as for the original integrals.

We consider operators of the form

$$
A f\left(\alpha_{j}\right)=\sum_{\ell \in I} K\left(\alpha_{j}, \alpha_{\ell}\right) f\left(\alpha_{\ell}\right) h^{2}
$$

acting on grid functions $f$. The kernel $K$ will be related to

$$
G_{h}^{\pi}\left(x_{j}-x_{\ell}\right)=G_{h}^{\pi}\left(x\left(\alpha_{j}\right)-x\left(\alpha_{\ell}\right)\right) .
$$

In order to say that certain operators gain derivatives in $L_{h}^{2}$, we use the operator $E$ defined in (4.16), representing an absolute derivative in a discrete sense. Thus if $A$ is an operator of type (4.2) such that $|a(x, k ; h)| \leq C\left(1+|k|^{2}\right)^{-n / 2}$ for some $n \geq 0$, with the same estimate for $x$-derivatives of $a$, then $A E^{n}$ is bounded on $L_{h}^{2}$, uniformly in $h$, according to Lemma 4.2, i.e., $A$ gains $n$ derivatives. When $A E^{n}$ is bounded in this sense for $n \geq 0$, we will say that $A$ is of order $-n$ as an operator on $L_{h}^{2}$. The following theorem summarizes properties of discrete operators associated with $G_{h}$ which will be needed for the stability estimates for the scheme.

Theorem 5.1. Suppose that the regularized Green's function $G_{h}$ satisfies the assumptions (2.1) -(2.3) and $\hat{G}_{h}(k)<0$. Let $A$ be the operator

$$
A f\left(\alpha_{j}\right)=\sum_{\ell \in I} G_{h}^{\pi}\left(x_{j}-x_{\ell}\right) f\left(\alpha_{\ell}\right) h^{2} .
$$

Then $A$ is of order -1, i.e., $A E$ is bounded on $L_{h}^{2}$ uniformly in $h$. Specifically, $A$ has the form $A=-A^{(1)}-A^{(2)}$, where $A^{(1)}$ has order $-1, A^{(2)}$ has order -2 , and 
$A^{(1)}$ is an operator of type (4.2) whose symbol $a_{1}(\alpha, k ; h)$ is of class $S^{-1}$, in the sense of (4.10), and also satisfies, for some $c_{1}>0$,

$$
a_{1}(\alpha, k ; h) \geq c_{1}\left(1+|k|^{2}\right)^{-1 / 2}
$$

More generally, suppose $\tilde{x}_{j}$ approximates $x_{j}=x\left(\alpha_{j}\right)$, with $\left|\tilde{x}_{j}-x_{j}\right| \leq c_{0} h$ for each $j$, and $\tilde{x}_{j}-x_{j}$ is periodic. Let $\mu\left(\alpha, \alpha^{\prime}\right)$ be an arbitrary smooth, periodic function, and let $A_{m}$ be the operator of the form (5.1) which has the kernel $\mu\left(\alpha_{j}, \alpha_{\ell}\right) D_{x}^{m} G_{h}^{\pi}\left(\tilde{x}_{j}-\tilde{x}_{\ell}\right)$, where $D_{x}^{m}$ is a derivative of order $|m|$ for multi-index $m$. Then $A_{m}$ is of order -1 for $m=0$ and order 0 for $|m|=1$. For $|m|=2$, the operator $h A_{m}$ is of order 0 . In the special case that $\mu=0$ on the diagonal $\alpha=\alpha^{\prime}$, the operator $A_{m}$ has order $|m|-2$ for $0 \leq|m| \leq 2$. Also for arbitrary $\mu$ the operator with kernel $\mu \partial G_{h}^{\pi}\left(x_{j}-x_{\ell}\right) / \partial n$ is of order -1 , where $n$ denotes either normal vector $n\left(x_{j}\right)$ or $n\left(x_{\ell}\right)$.

We also need a more special result concerning the discrete derivative of $G_{h}$, showing an extra gain of a derivative due to a smooth factor $\mu$ which vanishes on the diagonal.

Theorem 5.2. With $G_{h}$ as above, let $D_{h}$ be a first order derivative operator as in (1.14) -(1.16), and let $\mu\left(\alpha, \alpha^{\prime}\right)$ be smooth and periodic, with $\mu=0$ when $\alpha=\alpha^{\prime}$. Then the following two operators are bounded on $L_{h}^{2}$ uniformly in $h$, where both $D_{h}$ and $E$ act with respect to $\alpha_{\ell}$ :

$$
\begin{gathered}
B_{1} f\left(\alpha_{j}\right)=\sum_{\ell \in I} D_{h} G_{h}^{\pi}\left(x_{j}-x_{\ell}\right) \mu\left(\alpha_{j}, \alpha_{\ell}\right) E f\left(\alpha_{\ell}\right) h^{2}, \\
B_{2} f\left(\alpha_{j}\right)=\sum_{\ell \in I} E^{2} G_{h}^{\pi}\left(x_{j}-x_{\ell}\right) \mu\left(\alpha_{j}, \alpha_{\ell}\right) f\left(\alpha_{\ell}\right) h^{2}
\end{gathered}
$$

To begin deriving these facts, we first split the Green's function into a far-field part and a local part, in order to focus attention on the latter. We wish to restrict the local analysis to a small neighborhood of the singularity in which the coordinate mapping is well approximated by its linearization. Let $J(\alpha)$ be the Jacobian matrix $\partial x / \partial \alpha$ at $\alpha$. Because of (1.13) and the smoothness of $x(\alpha)$, there is some $\delta_{0}$ small enough so that $\delta_{0}<\pi / 2$ and

$$
\left|J(\alpha)\left(\alpha-\alpha^{\prime}\right)\right| / 2 \leq\left|x(\alpha)-x\left(\alpha^{\prime}\right)\right| \leq 2\left|J(\alpha)\left(\alpha-\alpha^{\prime}\right)\right|, \quad\left|\alpha-\alpha^{\prime}\right| \leq \delta_{0} .
$$

Also from (1.13) there exists $r_{0}>0$ with $r_{0}<\pi / 2$ so that $\left|x(\alpha)-x\left(\alpha^{\prime}\right)\right| \leq r_{0}$ implies $\left|\alpha-\alpha^{\prime}\right| \leq \delta_{0}$. We can also assume that $\left|J(\alpha)\left(\alpha-\alpha^{\prime}\right)\right| \leq r_{0}$ implies $\left|\alpha-\alpha^{\prime}\right| \leq \delta_{0}$. We now choose a cut-off function $\zeta: \mathbf{R}^{3} \rightarrow \mathbf{R}$ so that $\zeta(x)=1$ for $x$ near 0 and $\zeta(x)=0$ for $|x|>r_{0}$. Thus (5.6) holds whenever $x(\alpha)-x\left(\alpha^{\prime}\right)$ is within the support of $\zeta$. We write the regularized Green's function as $G_{h}=\zeta G_{h}+(1-\zeta) G_{h}$. The periodic version is then split as $G_{h}^{\pi}=G_{h 0}^{\pi}+G_{h \infty}^{\pi}$, as in Theorem 2.2. The far-field part $G_{h \infty}^{\pi}$ is smooth and periodic. Consequently a discrete integral operator with kernel $D^{m} G_{h \infty}^{\pi}\left(x_{j}-x_{\ell}\right)$ is $L_{h}^{2}$-bounded, uniformly in $h$, for $D^{m}$ an $x$-derivative of any order $m$. The local term $G_{h 0}^{\pi}$ when evaluated on the surface is

$$
G_{h 0}^{\pi}\left(x(\alpha)-x\left(\alpha^{\prime}\right)\right)=G_{h}\left(x(\alpha)-x\left(\alpha^{\prime}\right)\right) \zeta\left(x(\alpha)-x\left(\alpha^{\prime}\right)\right)
$$

when $\left|\alpha_{\nu}^{\prime}-\alpha_{\nu}\right| \leq \pi$; the remaining terms in the sum are zero because of the small support of $\zeta$. Thus the boundedness properties of a discrete operator with kernel $D^{m} G_{h}^{\pi}\left(x_{j}-x_{\ell}\right)$ reduce to consideration of $D^{m}\left(G_{h 0}\right)\left(x_{j}-x_{\ell}\right)$, where $G_{h 0}(x)=$ $G_{h}(x) \zeta(x)$. 
We now consider the boundedness properties of the operator (5.2). Because of the above remarks, we replace $G_{h}^{\pi}$ by $G_{h 0}^{\pi}$. We will make a Taylor expansion of the kernel, which will enable us to use the representation (4.20) for the resulting terms. We treat $x_{j}-x_{\ell}$ as a perturbation of its linearization $y_{j \ell}=J_{j}\left(\alpha_{j}-\alpha_{\ell}\right)$, where $J_{j}=J\left(\alpha_{j}\right)$. Thus we can write $G_{h 0}^{\pi}\left(x_{j}-x_{\ell}\right)$ as an expansion in terms $D_{x}^{m} G_{h 0}^{\pi}\left(y_{j \ell}\right)\left(z_{j \ell}\right)^{m}$, summed over $m$ with remainder, where $z_{j \ell}=x\left(\alpha_{j}\right)-x\left(\alpha_{\ell}\right)-y_{j \ell}$, and $m$ is a multi-index. We can further expand $z_{j \ell}$ in powers of $\alpha_{j}-\alpha_{\ell}$, quadratic or higher. We then have a linear combination of terms, a typical one being, for $\left|\alpha_{\ell \nu}-\alpha_{j \nu}\right|<\pi$

$$
D_{x}^{m} G_{h 0}^{\pi}\left(y_{j \ell}\right)\left(\alpha_{j}-\alpha_{\ell}\right)^{p}=D_{x}^{m} G_{h 0}^{\pi}\left(J_{j}\left(\alpha_{j}-\alpha_{\ell}\right)\right)\left(\alpha_{j}-\alpha_{\ell}\right)^{p} .
$$

Here $p$ is another multi-index, with $|p| \geq 2|m|$. The discrete integral operator with (5.8) as kernel, extended periodically, has the form (4.20), and therefore (4.22), with symbol given by (4.24). Thus we can derive boundedness properties of this operator by estimating the Fourier transform of the kernel $\alpha^{p} D_{x}^{m} G_{h 0}\left(J_{j} \alpha\right)$ with respect to $\alpha$. The relevant estimates are stated in the following lemma. The rapid decay for $k h$ large is needed for the convergence of the sum (4.24). These estimates and the previous remarks show that the operator with kernel (5.8) gains $|p|+1-|m|$ derivatives, i.e., it is of order $|m|-|p|-1$ on $L_{h}^{2}$ in the sense defined above.

Lemma 5.3. Let $K_{m p}(\alpha)=\alpha^{p} D_{x}^{m} G_{h 0}(J \alpha)$, where $J$ is the Jacobian matrix at some fixed $\alpha_{0}$. Assume that $|p|+1 \geq|m|$. Then the Fourier transform $\hat{K}_{m p}$ satisfies the following estimates uniformly in $h$ :

$$
\left|\hat{K}_{m p}(k)\right| \leq \begin{cases}C, & |k| \leq 1, \\ C|k|^{|m|-|p|-1}, & 1 \leq|k| \leq C_{0} / h, \\ C h^{-|m|+|p|+1}|k h|^{-n}, & |k| \geq 1 / h,\end{cases}
$$

with $n$ large. Similar estimates hold for the derivatives of $\hat{K}_{m p}$ with respect to $\alpha_{0}$. The same estimates hold for $|k| \geq 1$ if $G_{h 0}$ is replaced by $G_{h}$. For $p=0$ and $|m|=2$, we have the same estimate for $|k|>1 / h$, and $\left|\hat{K}_{m p}(k)\right| \leq C h^{-1}$ for $|k| \leq C_{0} / h$.

In the rest of this section we present the proof of the Lemma 5.3, then the proofs of Theorems 5.1 and 5.2, and one final lemma.

Proof of Lemma 5.3. In estimating this transform it is helpful to note that the $x$ derivatives on $G_{h}$ can be replaced by $\alpha$-derivatives, as we now explain. First we extend the Jacobian $J: \mathbf{R}^{2} \rightarrow \mathbf{R}^{3}$ at $\alpha_{0}$ to a nonsingular operator $\tilde{J}: \mathbf{R}^{3} \rightarrow \mathbf{R}^{3}$ by defining

$$
\tilde{J}\left(\alpha_{1}, \alpha_{2}, \alpha_{3}\right)=J\left(\alpha_{1}, \alpha_{2}\right)+\alpha_{3} N
$$

where $N=X_{1} \times X_{2}$ and $X_{1}, X_{2}$ are the tangent vectors at $\alpha_{j}$, as before. Now since $G_{h}$ is radial, $\left(\nabla G_{h}\right)(J \alpha)$ has the direction of $J \alpha$ for $\alpha \in R^{2}$, and thus is perpendicular to $N$, so that $\left(\nabla G_{h}\right)(J \alpha)=\left(G_{h} \circ J\right)_{\alpha_{1}} X_{1}^{*}+\left(G_{h} \circ J\right)_{\alpha_{2}} X_{2}^{*}$. Thus $D_{x} G_{h}$ can be replaced with $D_{\alpha}\left(G_{h} \circ J\right)$. Next we note that the matrices of second derivatives are related by $D_{\alpha}^{2}\left(G_{h} \circ \tilde{J}\right)=\tilde{J}^{*}\left(D_{x}^{2} G_{h}\right) \tilde{J}$. This shows that $D_{x}^{2} G_{h}$ can be expressed in $\alpha$-derivatives of $G_{h} \circ \tilde{J}$ for $\alpha \in R^{3}$. However, for $\alpha_{3}=0$ we have $\partial\left(G_{h} \circ \tilde{J}\right) / \partial \alpha_{3}=0$, and the same is true for the second partials in $\alpha_{3}, \alpha_{1}$ or $\alpha_{3}, \alpha_{2}$. Also the second partial in $\alpha_{3}, \alpha_{3}$ can be rewritten in $\alpha_{1}, \alpha_{2}$-derivatives plus a multiple of $\psi_{h} \circ \tilde{J}$, since $\Delta G_{h}=\psi_{h}$. Thus we have reduced the second 
$x$-derivatives to $\alpha_{1}, \alpha_{2}$-derivatives plus the $\psi_{h}$ term. For higher derivatives, we can convert $D_{x}^{m} G_{h}(\tilde{J} \alpha)$ to $D_{\alpha}^{m}\left(G_{h} \circ \tilde{J}\right)$ for $\alpha \in \mathbf{R}^{3}$, and then repeat the above process, converting to $\alpha_{1}, \alpha_{2}$-derivatives, until a term with $\psi_{h}$ appears. In summary, a term of the form $D_{x}^{m} G_{h}(J \alpha)$ with $\alpha \in R^{2}$ can be replaced by a sum of terms of the form $D_{\alpha}^{m}\left(G_{h} \circ J\right)$, with $\alpha_{1}, \alpha_{2}$-derivatives only, and $D_{x}^{m-2} \psi_{h}(J \alpha)$.

We first estimate the transform of $F_{m p}(\alpha)=\alpha^{p} D_{\alpha}^{m}\left(G_{h}(J \alpha)\right)$. (We ignore the cut-off $\zeta$ for now and account for its effect later.) First, since $\Delta G_{h}=\psi_{h}$, we have $\hat{G}_{h}(k)=-\hat{\psi}_{h}(k) /|k|^{2}=-\hat{\psi}(k h) /|k|^{2}$ for the 3-D transforms. Now, as in \$2 we obtain the two-dimensional transform of $G_{h} \circ J$ from

$$
\left(G_{h} \circ J\right)^{\wedge}(k)=C \int_{-\infty}^{\infty} \hat{G}_{h}(M(k, \ell)) d \ell=C \int_{-\infty}^{\infty}|M(k, \ell)|^{-2} \hat{\psi}(M(k, \ell) h) d \ell,
$$

where $k \in \mathbf{R}^{2}$ and $M=\left(\tilde{J}^{*}\right)^{-1}$, with $\tilde{J}$ as above. To obtain the transform of $\alpha^{p} D_{\alpha}^{m}\left(G_{h}(J \alpha)\right)$, we need to multiply this by $(i k)^{m}$ and then apply $\left(i \partial_{k}\right)^{p}$. We obtain various terms where e.g., $q$ derivatives apply to $\hat{\psi}$ and the remaining $|p|-q$ apply to $k^{m}|M(k, \ell)|^{-2}$. It is important that $|M(k, \ell)|^{2}$ depends on $\ell$ only through an $\ell^{2}$ term; this follows from the definition of $\tilde{J}$. Thus $\partial_{k}|M(k, \ell)|^{2}$ in linear in $k$, without $\ell$ dependence. The resulting term has the form

$$
C \int_{-\infty}^{\infty} k^{s} h^{q}|M(k, \ell)|^{-n}\left(\partial^{q} \hat{\psi}\right)(M(k, \ell) h) d \ell
$$

with $n \geq 2$, and the total degree in $k$ has been lowered by $|p|-q$, i.e., $s-n=$ $|m|-2-|p|+q$. If we let $\kappa=|k h|$ and $\xi=\ell h$, and convert the integral to $\xi$, we see that the integral is dominated by

$$
C|k|^{s} h^{q+n-1} \int_{-\infty}^{\infty}\left(\kappa^{2}+\xi^{2}\right)^{-n / 2}\left(1+\kappa^{2}+\xi^{2}\right)^{-N / 2} d \xi
$$

for some large $N$; we have used the smoothness of $\psi$. We first estimate this assuming $k \neq 0$ and $|k h| \leq C_{0}$. In this case we drop the last factor in the integral (the resulting integral exists since $n \geq 2$ ) and convert the integral to $\xi / \kappa$. We find that the integral is of order $\kappa^{-n+1}$, and consequently the entire term above is bounded by $C|k|^{s} h^{q+n-1} \kappa^{-n+1}=|k|^{|m|-|p|-1}\left(|k h|^{q}\right)$, and in summary

$$
\left|\hat{F}_{m p}(k)\right| \leq C|k|^{|m|-|p|-1}, \quad|k h| \leq C_{0}, \quad k \neq 0 .
$$

On the other hand, for $|k h| \geq 1$, we return to 5.13 , drop the 1 in the second factor, and find that the integral is of order $|\kappa|^{-(n+N-1)}$. The expression (5.13) is then bounded by $C|\kappa|^{s-(n+N-1)} h^{q+n-1-s}=C|\kappa|^{s-(n+N-1)} h^{-|m|+|p|+1}$. The power of $\kappa=|k h|$ can be made large negative by taking $N$ large, and we obtain

$$
\left|\hat{F}_{m p}(k)\right| \leq C h^{-|m|+|p|+1}|k h|^{-n}, \quad|k h| \geq 1 .
$$

We need a similar estimate for the transform of $P_{m p}(\alpha)=\alpha^{p}\left(D_{x}^{m-2} \psi_{h}\right)(J \alpha)$. Omitting the $\alpha^{p}$ factor for the moment, we have

$$
\left(\left(D_{x}^{m-2} \psi_{h}\right) \circ J\right)^{\wedge}(k)=C \int_{-\infty}^{\infty}|M(k, \ell)|^{m-2} \hat{\psi}(M(k, \ell) h) d \ell .
$$


To obtain the transform of $P_{m p}$, we apply $\partial_{k}^{p}$ to the above. If $q$ derivatives apply to the $\hat{\psi}$ factor and $|p|-q$ apply to the first factor, we obtain an expression

$$
\int_{-\infty}^{\infty} \Phi(k, \ell) h^{q}\left(\partial^{q} \hat{\psi}\right)(M(k, \ell) h) d \ell
$$

where $\Phi$ is homogeneous of degree $s=|m|-2-|p|+q$. We now rewrite this with $\kappa=|k h|, \xi=\ell h$ and estimate by

$$
h^{q-s-1} \int_{-\infty}^{\infty}\left(\kappa^{2}+\xi^{2}\right)^{s / 2}\left(1+\kappa^{2}+\xi^{2}\right)^{-N / 2} d \xi
$$

for large $N$. For $k \neq 0$ and $\kappa=|k h| \leq C_{0}$ we replace the integrand in (5.18) by $\left(1+\kappa^{2}+\xi^{2}\right)^{-(N-s) / 2}$. The integral is $O(1)$, and the term is of order $h^{q-s-1}=$ $h^{-|m|+|p|+1}$; that is,

$$
\left|\hat{P}_{m p}(k)\right| \leq C h^{-|m|+|p|+1}, \quad|k h| \leq C_{0}, \quad k \neq 0 .
$$

If the exponent of $h$ is $\geq 0$, we can majorize by $C|k|^{|m|-|p|-1}$, as in the estimate (5.14) for $\hat{F}_{m p}$. On the other hand, for $|k h| \geq 1$, we drop the 1 in the second factor in (5.18), and find that the integral is of order $\kappa^{-n}$ for large $n$, so that

$$
\left|\hat{P}_{m p}(k)\right| \leq C h^{-|m|+|p|+1}|k h|^{-n}, \quad|k h| \geq 1 .
$$

Next we consider the cut-off function $\zeta$. We first show that the difference in the transform due to $\zeta$ is negligible for $|k| \geq 1$, i.e., we show that the transform of

$$
Q_{m p}(\alpha)=\alpha^{p} D_{x}^{m} G_{h}(J \alpha) \cdot(1-\zeta(J \alpha))
$$

is rapidly decreasing for large $k$. If we apply $D_{\alpha}^{s}$ to $Q_{m p}$ with $s$ of large degree, we can estimate using the chain rule and the fact that $\left|D_{x}^{\ell} G_{h}(x)\right| \leq C|x|^{-\ell-1}$ for $|x| \geq h$. We find that $\left|D_{\alpha}^{s} Q_{m p}(\alpha)\right| \leq C|\alpha|^{|p|-|m|-1-|s|}$, and of course $Q_{m p}(\alpha)=0$ for $\alpha$ near 0 and for $\alpha$ large. Thus $D_{\alpha}^{s} Q_{m p}$ is bounded in $L^{1}$ independent of $h$ for $|s|$ large. This implies that $\left|\hat{Q}_{m p}(k)\right| \leq C|k|^{-N}$ for $|k| \geq 1$ with $N$ large. This estimate is small enough that it will not affect the estimates already obtained for $|k| \geq 1$ when $F_{m p}, P_{m p}$ are multipled by $\zeta(J \alpha)$. There are additional terms where $\zeta$ is differentiated, but these terms vanish near 0 ; consequently their derivatives are bounded independent of $h$, and the transform is again rapidly decreasing.

It remains to estimate the transforms of $F_{m p}(\alpha) \zeta(J \alpha)$ and $P_{m p}(\alpha) \zeta(J \alpha)$ for $|k| \leq 1$. In the first case the transform is

$$
(2 \pi)^{-1} \int \alpha^{p} D_{\alpha}^{m}\left(G_{h}(J \alpha)\right) e^{-i k \alpha} \zeta(J \alpha) d^{2} \alpha .
$$

We can integrate by parts so that the $m$ derivatives apply to $\alpha^{p} e^{-i k \alpha} \zeta(J \alpha)$. The result is an integrand bounded by $\left|G_{h}(J \alpha)\right|$, for $|k| \leq 1$, over the support of $\zeta$. Since $\left|G_{h}(x)\right| \leq h^{-1}$ and $\left|G_{h}(x)\right| \leq|x|^{-1}$ for $|x| \geq h$, we can easily show that this last integral is independent of $h$. For the case of $P_{m p} \zeta$ we cannot integrate by parts because we have $x$-derivatives; however, we can use the scaling of $\psi_{h}$ in $h$ to show that the transform is bounded by $C h^{-|m|+|p|+1}$.

Finally, combining the estimates (5.14), (5.15), (5.19), (5.20) and the last two paragraphs, we obtain the estimates stated for $\hat{K}_{m p}$. It can be checked that derivatives in $\alpha_{0}$ do not seriously affect the estimates, so that the same bounds can be obtained in this case; the dependence on $\alpha_{0}$ is through $J=J\left(\alpha_{0}\right)$ in $K_{m p}$ and therefore through $M$ in the transform. 
Proof of Theorem [5.1] We first consider the operator (5.2). As explained before, we reduce consideration to $G_{h 0}^{\pi}$ and form the Taylor expansion in terms (5.8). The operator with kernel (5.8) has the form (4.20), (4.22), with symbol given by (4.24), and it follows from the estimates of Lemma 5.3 and from Lemma 4.2 that this operator is bounded of order $|m|-|p|-1$. The first term, with $m=p=0$, is of order -1 and the others -2 or less, since $|p| \geq 2|m|$. It remains to show that the remainder is of order -2 . Suppose we carry the expansion for $G_{h 0}^{\pi}$ to terms with $|m|<N$ and the one for $z_{j \ell}$ to terms of degree $\leq N$ in $\alpha$. Then we have two kinds of remainders: One has the form $F_{N}\left(\alpha_{j}, \alpha_{\ell}\right)\left(z_{j \ell}\right)^{N}$, where $F_{N}$ is an average of $D_{x}^{N} G_{h 0}^{\pi}$ over the line from $y_{j \ell}$ to $x_{j}-x_{\ell}$. The other type of remainder, from expanding $z_{j \ell}$, is $D_{x}^{m} G_{h 0}^{\pi}\left(y_{j \ell}\right)\left(\alpha_{j}-\alpha_{\ell}\right)^{p} g\left(\alpha_{j}-\alpha_{\ell}\right)$, where $g$ is some smooth function and $|p| \geq N+2|m|-1$. It is routine to check that derivatives up to order $N-1$ of either remainder with respect to $\alpha_{\ell}$, treated as a continuous variable, are uniformly bounded. We can use this fact to estimate the remainders crudely. We need to show that $R E^{2}$ is bounded as an operator on $L^{2}$, where $R$ is the operator corresponding to a remainder term. If $R$ has kernel $R\left(\alpha_{j}, \alpha_{\ell}\right)$, we can regard $\left(R E^{2} f\right)\left(\alpha_{j}\right)$ as the inner product of $R\left(\alpha_{j}, \cdot\right)$ with $E^{2} f$. Since $E^{2}$ is self-adjoint, this will be uniformly bounded by $|f|_{L^{2}}$ provided $E^{2} R\left(\alpha_{j}, \cdot\right)$ is bounded uniformly in $\alpha_{j}, \alpha_{\ell}$, where $E^{2}$ acts on the $\alpha_{\ell}$ variable. It can be shown that the spectral derivative $E^{2} R$ is bounded by derivatives $D_{\alpha}^{m} R$ for $|m| \leq 5$ (cf. 31], pp. 250-4), and thus the operator $R E^{2}$ is bounded provided $N \geq 6$.

The decomposition $A=-A^{(1)}-A^{(2)}$ essentially follows from separating out the leading term in the expansion, namely $G_{h 0}^{\pi}\left(J\left(\alpha_{j}\right)\left(\alpha_{j}-\alpha_{\ell}\right)\right)$. This term contributes to $A$ an operator of the form (4.22) whose symbol is given by (4.24) with $K$ replaced by $G_{h 0} \circ J$. Since $\hat{G}_{h}(k)<0$, and since $\left(G_{h 0} \circ J\right)^{\wedge}(k)-\left(G_{h} \circ J\right)^{\wedge}(k)$ decreases rapidly for large $k$, as seen below (5.21), we will take $A^{(1)}$ to be a similar operator with kernel $K$ whose transform agrees with $\left(-G_{h} \circ J\right)^{\wedge}(k)$ for large $k$. Let $\chi:[0, \infty) \rightarrow$ $[0,1]$ be a smooth function with $\chi(r)=1$ for $r \leq 1$ and $\chi(r)=0$ for $r \geq 2$. We define the kernel $K$ by $\hat{K}(k)=\chi(|k|)+(1-\chi(|k|))\left(-G_{h} \circ J\right)^{\wedge}(k)$. Then $A^{(2)}=-A-A^{(1)}$ has order -2 . We proceed to verify the properties of the symbol $a_{1}$ of $A^{(1)}$, which is the sum (4.24). The estimates of Lemma 5.3 show that the sum converges; the sum is positive since the terms are. To show that $a_{1}$ is bounded below by $|k|^{-1}$ it is enough to show that $-\left(G_{h} \circ J\right)^{\wedge}(k) \geq c_{1}|k|^{-1}$ for $1 \leq|k| \leq C_{0} / h$. This can be seen by rewriting (5.11) with $(k \cdot \ell)=|k|(\theta, \xi)$ as

$$
-\left(G_{h} \circ J\right)^{\wedge}(|k| \theta)=C|k|^{-1} \int_{-\infty}^{\infty}|M(\theta, \xi)|^{-2} \hat{\psi}(M(\theta, \xi)|k| h) d \xi
$$

and recalling that $\hat{\psi}$ is positive and smooth. The estimate for $D_{\alpha}^{s} a_{1}$ follows from the lemma. With regard to the $k$-difference in (4.10), we note that $i \partial\left(G_{h} \circ J\right)^{\wedge} / \partial k_{\nu}$ is the transform of $\alpha_{\nu} G_{h} \circ J$. This transform decays like $|k|^{-2}$, according to the lemma. The estimate for $\Delta_{(k)} D_{\alpha}^{s} a_{1}$ follows, bounding $k$-differences by derivatives for $|k| \geq 2$.

Next we consider the other operators, first ignoring the smooth factor $\mu$ and assuming $\tilde{x}_{j}=x_{j}$. For the operator with kernel $D_{x} G_{h}^{\pi}\left(x_{j}-x_{\ell}\right)$ the argument above applies for the boundedness, except that the first term in the expansion now has $m=1, p=0$, so that the leading operator has order 0 . A similar remark applies to $D_{x}^{2} G_{h}^{\pi}$. If the factor $\mu\left(\alpha_{j}, \alpha_{\ell}\right)$ is included, we expand it in the second variable in powers of $\alpha_{j}-\alpha_{\ell}$ and incorporate this into the previous expansion, so that there is 
no qualitative change. For $\partial G_{h}^{\pi} / \partial n\left(\alpha_{\ell}\right)=-n\left(\alpha_{\ell}\right) \cdot \nabla G_{h}^{\pi}$, we use a similar expansion for $n\left(\alpha_{\ell}\right)$ in powers of $\alpha_{j}-\alpha_{\ell}$. The leading term is $-n\left(\alpha_{j}\right) \cdot \nabla G_{h}\left(J\left(\alpha_{j}\right)\left(\alpha_{j}-\alpha_{\ell}\right)\right)$, and this is zero since $G_{h}$ is radial and the range of $J\left(\alpha_{j}\right)$ is tangent at $\alpha_{j}$; the remaining operator is of order -1 . In case $\mu=0$ on the diagonal, the leading term in the expansion of $\mu$ is zero. The other terms have at least one factor of $\left(\alpha_{j}-\alpha_{\ell}\right)$, effectively raising the index $p$ by 1 in each term, and the order of the operator is changed as stated.

Finally we consider the effect of perturbing $x_{j}$ to $\tilde{x}_{j}$. First, using the pointwise estimates (2.4) for derivatives of $G_{h}$, we can show that

$$
\sum_{\ell \in I}\left|D_{x}^{m} G_{h}^{\pi}\left(\tilde{x}_{j}-\tilde{x}_{\ell}\right)\right| h^{2} \leq \begin{cases}C, & |m|=0 \\ C|\log h|, & |m|=1 \\ C h^{-|m|+1}, & |m|=2,\end{cases}
$$

for $\left|\tilde{x}_{j}-x_{j}\right| \leq c_{0} h$, and the same for the sum over $j$; see e.g., Lemma 5 of [16] or Lemma 3.2 of [7]. It follows from Young's inequality that the discrete integral operator with kernel $D_{x}^{m} G_{h}^{\pi}\left(\tilde{x}_{j}-\tilde{x}_{\ell}\right)$, regarded an operator from $L_{h}^{2}$ to itself, has norm bounded by the right side of (5.24) (e.g., cf. [7], pp. 13-14). The same is true if the kernel is multiplied by a uniformly bounded factor such as $\mu$.

Now suppose the operator with kernel $D_{x} G_{h}^{\pi}\left(x_{j}-x_{\ell}\right)$ is perturbed by replacing $x_{j}, x_{\ell}$ by $\tilde{x}_{j}, \tilde{x}_{\ell}$. The difference of the two kernels can be written as an average of $D_{x}^{2} G_{h}^{\pi}$, evaluated along the line from $x_{j}-x_{\ell}$ to $\tilde{x}_{j}-\tilde{x}_{\ell}$, multiplied by $\left(\tilde{x}_{j}-x_{j}\right)-\left(\tilde{x}_{\ell}-x_{\ell}\right)$. If we assume that $\max \left|\tilde{x}_{j}-x_{j}\right| \leq C_{0} h$, then we find, using the above with $|m|=2$, that the operator on $L_{h}^{2}$ corresponding to this difference is bounded in norm by $C h^{-1} \cdot h=C$. A similar argument works for the kernel $\mu D_{x} G_{h}^{\pi}\left(\tilde{x}_{j}-\tilde{x}_{\ell}\right)$, and this verifies the statement in the theorem regarding this operator. It also improves the statement made above for bounds on $L_{h}^{2}$ operators in the case $|m|=1$, replacing $O(|\log h|)$ by $O(1)$. That is, the norm of the operator on $L^{2}$ with kernel $\mu D_{x}^{m} G_{h}^{\pi}\left(x_{j}-x_{\ell}\right)$ is $O(1)$ if $|m|=0,1$, and $O\left(h^{-|m|+1}\right)$ if $m \geq 2$. We can now use this improved statement with $|m|=1$ to verify the assertion that $\mu G_{h}^{\pi}\left(\tilde{x}_{j}-\tilde{x}_{\ell}\right)$ gains one derivative: the error due to the change in the $x$ 's is found to be $O(h)$ as an operator on $L^{2}$, and $h E$ is a bounded operator on $L^{2}$. We can treat the operator $h \mu D_{x}^{2} G_{h}^{\pi}$ similarly.

In the special case with $\mu=0$ on the diagonal, the pointwise estimates for the kernel are better, and we can show that the operator $\mu D_{x}^{2} G_{h}^{\pi}\left(\tilde{x}_{j}-\tilde{x}_{\ell}\right)$ is bounded on $L^{2}$ by an argument very similar to that above. We can then use that fact to show that $\mu D_{x} G_{h}^{\pi}\left(\tilde{x}_{j}-\tilde{x}_{\ell}\right)$ gains one derivative. In turn, this last fact can be used to show that $\mu G_{h}^{\pi}\left(\tilde{x}_{j}-\tilde{x}_{\ell}\right)$ gains two derivatives.

Proof of Theorem 5.2. We prove that $B_{1}$ is bounded and then remark on $B_{2}$. Let us say that $D_{h}$ acts in the $r$-direction, so that e.g., $D_{h} g$ has transform $h^{-1} \sigma\left(k_{r} h\right) \ddot{g}(k)$ for $k \in I$. We will write $\eta(k)=(1+|k|)^{1 / 2}$ for the symbol of $E$. Regarding the sum as an inner product, we can rewrite the operator as

$$
B_{1} f\left(\alpha_{j}\right)=\sum_{\ell \in I} G_{h}^{\pi}\left(x_{j}-x_{\ell}\right) D_{h, r}^{*}\left(\mu\left(\alpha_{j}, \alpha_{\ell}\right) E f\left(\alpha_{\ell}\right)\right) h^{2} .
$$

We will compare this with

$$
B_{0} f\left(\alpha_{j}\right)=\sum_{\ell \in I} G_{h}^{\pi}\left(x_{j}-x_{\ell}\right) \mu\left(\alpha_{j}, \alpha_{\ell}\right) D_{h, r}^{*} E f\left(\alpha_{\ell}\right) h^{2} .
$$


The previous theorem tells us that $G_{h}^{\pi} \mu$ gains two derivatives, since $\mu=0$ on the diagonal, and it follows that $B_{0}$ is bounded on $L^{2}$, independent of $h$. (We think of $D_{h, r}^{*}$ as $E$ times a bounded operator.) Thus we need only treat the difference of the two operators.

To express $\left(B_{1}-B_{0}\right) f$, we write $f$ and $\mu$ in Fourier sums:

$$
f\left(\alpha_{\ell}\right)=\sum_{k \in I} \ddot{f}(k) e^{i k \ell h}, \quad \mu\left(\alpha_{j}, \alpha_{\ell}\right)=\sum_{\nu \in I} \ddot{\mu}\left(\alpha_{j}, \nu\right) e^{i \nu \ell h} .
$$

Since $\mu$ is assumed smooth, $\ddot{\mu}\left(\alpha_{j}, \nu\right)$ decays rapidly in $\nu$. Then

$$
\left(B_{1}-B_{0}\right) f\left(\alpha_{j}\right)=\sum_{\nu, \ell} \ddot{\mu}\left(\alpha_{j}, \nu\right)|\nu| e^{i \nu \ell h} G_{h}^{\pi}\left(x_{j}-x_{\ell}\right) \sum_{k} b(\nu, k) \eta(k) \ddot{f}(k) e^{i k \ell h} h^{2},
$$

where we have set $h^{-1}\left(\bar{\sigma}\left(\left(\nu_{r}+k_{r}\right) h\right)-\bar{\sigma}\left(k_{r} h\right)\right)=|\nu| b(\nu, k)$. Since we assume $\sigma$ is continuous with bounded derivative, we have $\left|\bar{\sigma}\left(\left(\nu_{r}+k_{r}\right) h\right)-\bar{\sigma}\left(k_{r} h\right)\right| \leq C h\left|\nu_{r}\right|$, so that $b(\nu, k)$ is uniformly bounded. We think of the $k$-sum as an operator on $f$ and write this as

$$
\left(B_{1}-B_{0}\right) f\left(\alpha_{j}\right)=\sum_{\nu, \ell} \ddot{\mu}\left(\alpha_{j}, \nu\right)|\nu| e^{i \nu \ell h} G_{h}^{\pi}\left(x_{j}-x_{\ell}\right)\left(E \Phi_{\nu} f\right)(\ell h) h^{2},
$$

where $\Phi_{\nu}$ is defined by $\left(\Phi_{\nu} f\right)^{*}(k)=b(\nu, k) \ddot{f}(k)$. The operator $\Phi_{\nu}$ is bounded on $L^{2}$ uniformly in $\nu$ as well as $h$. Finally, we can think of the above as a sum over $\nu$ of operators applied to $E \Phi_{\nu} f$, with kernel $\ddot{\mu}\left(\alpha_{j}, \nu\right)|\nu| e^{i \nu \ell h} G_{h}^{\pi}\left(x_{j}-x_{\ell}\right)$. Each such operator gains one derivative according to Theorem [5.1 i.e., when composed with $E$, it gives a bounded operator on $L^{2}$ for each $\nu$, with norm depending on the smoothness in $\left(\alpha_{j}, \alpha_{\ell}\right)$ of the factor $\ddot{\mu}\left(\alpha_{j}, \nu\right)|\nu| e^{i \nu \alpha_{\ell}}$. To sum over $\nu$, we need a bound for some high norm of the factor $e^{i \nu \alpha_{\ell}}$, and this bound grows with $\nu$. However, assuming $\mu$ is smooth enough, $\ddot{\mu}\left(\alpha_{j}, \nu\right)$ decays so rapidly in $\nu$ that the sum is bounded, and we can conclude that $B_{1}-B_{0}$ is bounded on $L^{2}$ independent of $h$.

It suffices to prove the second statement with $E^{2}$ replaced by $D_{h, r}, r=1,2$, since $E^{2}$ can be written in terms of these. For this we compare (5.5) with

$$
\sum_{\ell \in I} D_{h} G_{h}^{\pi}\left(x_{j}-x_{\ell}\right) \mu\left(\alpha_{j}, \alpha_{\ell}\right) D_{h}^{*} f\left(\alpha_{\ell}\right) h^{2} .
$$

The latter is bounded according to (5.4), and the difference can be treated as in the above argument.

Finally we need one more fact about sums with $D^{m} G_{h}^{\pi}$ :

Lemma 5.4. Suppose $\mu\left(\alpha, \alpha^{\prime}\right)$ is a smooth, periodic function. Let $\tilde{x}$ be a grid function approximating the coordinate mapping $x$ so that $\left|\tilde{x}\left(\alpha_{j}\right)-x\left(\alpha_{j}\right)\right| \leq c_{0} h$ for each $j$. Then the sum

$$
\sum_{\ell \in I} D^{m} G_{h}^{\pi}\left(\tilde{x}_{j}-\tilde{x}_{\ell}\right) \mu\left(\alpha_{j}, \alpha_{\ell}\right) h^{2}
$$

is bounded uniformly in $j$ and $h$, where $m$ is a multi-index with $|m|=1$, i.e., $D^{m}$ denotes any first derivative in $x$. If $\mu=0$ when $\alpha=\alpha^{\prime}$, then the sum is also uniformly bounded for any $m$ with $|m|=2$. 
Proof. The essential point is that, since $G_{h}$ is radial, $D G_{h}$ is odd in $x$ and $D^{2} G_{h}$ is even. The proofs of the two cases are similar; we prove the second. Only a small neighborhood of the diagonal matters; we assume $\mu$ is supported on such a neighborhood and replace $G_{h}^{\pi}$ with $G_{h}$. At first we assume $\tilde{x}$ is identical with $x$. For simplicity we take $j=0$ and assume $x_{0}=x\left(\alpha_{0}\right)=0$. Since $\mu(0, \alpha)=0$ at $\alpha=0$, we can write $\mu(0, \alpha)=\mu_{1}(\alpha)+\mu_{2}(\alpha)$, where $\mu_{1}$ is odd in $\alpha$ and $\mu_{2}=O\left(\alpha^{2}\right)$ as $\alpha \rightarrow 0$. Thus, if we replace $\mu$ by $\mu_{1}$ in the sum and average with the sum where $\ell$ is changed to $-\ell$, we get

$$
\frac{1}{2} \sum_{\ell \in I}\left[D^{2} G_{h}\left(x\left(\alpha_{\ell}\right)\right)-D^{2} G_{h}\left(x\left(-\alpha_{\ell}\right)\right)\right] \mu_{1}\left(\alpha_{\ell}\right) h^{2} .
$$

Since $x$ is smooth, $x(-\alpha)=-x(\alpha)+O\left(\alpha^{2}\right)$, and $D^{2} G_{h}\left(x\left(-\alpha_{\ell}\right)\right)=D^{2} G_{h}\left(-x\left(\alpha_{\ell}\right)\right)$ $+R_{3}$, where the remainder $R_{3}$ is $O\left(\alpha_{\ell}^{2}\right)$ times an average of $D^{3} G_{h}$ at points near $x\left(-\alpha_{\ell}\right)$. When we substitute this in the last sum, the $D^{2} G_{h}$ terms cancel because of the evenness property. We are left with the sum of $R_{3} \mu_{1}$. This is of order $\left|\alpha_{\ell}\right|^{-1}$ for $\ell \neq 0$, since $D^{3} G_{h}$ is of order $\left|\alpha_{\ell}\right|^{-4}$ and $\mu_{1}$ is of order $\left|\alpha_{\ell}\right|$. This corresponds to an integrable singularity in 2-D, and the sum is bounded independent of $h$. For the sum with $\mu_{2}$, we arrive more directly at a similar estimate, using $\mu_{2}=O\left(|\alpha|^{2}\right)$ and $D^{2} G_{h}=O\left(|\alpha|^{-3}\right)$.

To complete the proof, we estimate the change in the sum when $x$ is perturbed to $\tilde{x}$. Again with $j=0, x(0)=0$, the change in $D^{2} G_{h}$ is $O(h)$ times an average of $D^{3} G_{h}$ at points near $x\left(-\alpha_{\ell}\right)$. For some $C_{1},\left|\tilde{x}\left(\alpha_{\ell}\right)\right|>h$ and $\left|x\left(\alpha_{\ell}\right)\right|>h$ when $\left|\alpha_{\ell}\right|>C_{1} h$. Then for such $\ell$ we again use $D^{3} G_{h}=O\left(\left|\alpha_{\ell}\right|^{-4}\right)$ to bound the resulting sum by

$$
h \cdot \sum_{\ell \neq 0}\left|\alpha_{\ell}\right|^{-4}\left|\alpha_{\ell}\right| h^{2}=h^{3} \sum_{\ell \neq 0}|\ell h|^{-3}=C .
$$

The remaining $\ell$ 's are $O(1)$ in number, and it can be checked that their contribution to the sum is $O(1)$.

\section{The CONVERGEnce PRoOf}

We now prove that the numerical solution converges to the presumed smooth solution with error $O\left(h^{3}\right)$. As usual we separate consistency and stability, beginning with the former. Assuming an exact solution given, we first compare the velocity $v$, as computed by the scheme at one time from $x\left(\alpha_{j}, t\right), \phi\left(\alpha_{j}, t\right)$, with the exact velocity. First $X_{1}, X_{2}$ are found at $\alpha_{j}$ using the discrete operator $D_{h}$, and then $N, n, X_{1}^{*}, X_{2}^{*}$. The tangential part of $v$ is

$$
\nabla_{h}^{T} \phi=\sum_{k=1,2}\left(D_{h, k} \phi\right) X_{k}^{*}
$$

The full computed velocity is

$$
v=\nabla_{h}^{T} \phi+w n,
$$

where $w$ is found from the discrete integral equation (1.25), with the operator $\mathcal{K}_{h}$ defined by (1.26) and $f_{j}$ by (1.27). The following lemma, proved in $\$ 7$, concerns the solvability of (1.25).

Lemma 6.1. Given $x(\alpha, t)$ smooth for $0 \leq t \leq T$, the operator $I+2 \mathcal{K}_{h}$ is invertible for $h$ sufficiently small, and the norm of $\left(I+2 \mathcal{K}_{h}\right)^{-1}$ as an operator on $L_{h}^{2}$ is bounded uniformly in $h$ and $t$. 
It is evident that $\nabla_{h}^{T} \phi$ and $n$ differ by $O\left(h^{3}\right)$ from the exact values. To compare $w$ with $\phi_{n}$, determined by the harmonic extension of $\phi$, we note that $\phi_{n}$ satisfies the exact integral equation (1.3). The quadrature results in $\$ 3$ show that $\phi_{n}$ has truncation error $O\left(h^{3}\right)$ in the discrete version (1.25). Then Lemma 6.1 implies that $w-\phi_{n}=O\left(h^{3}\right)$ at least in $L_{h}^{2}$. Thus $v$ of (6.2) also differs by $O\left(h^{3}\right)$ from the actual velocity. A similar statement follows for Bernoulli's equation, and in summary the exact solution satisfies the following with $p=3$ :

$$
x_{t}=v+O\left(h^{p}\right), \quad \phi_{t}=\frac{1}{2}|v|^{2}-g x_{3}+O\left(h^{p}\right) \quad \text { in } L_{h}^{2} .
$$

It seems that $O\left(h^{3}\right)$ accuracy is not quite enough for simple treatment of the nonlinear terms in the stability estimates. For this reason we use a version of Strang's method (see [26], §5.6). That is, we construct a modification of the exact solution which matches the scheme to higher order, and then compare the computed solution with this modification. The following lemma (proved in 97 ) asserts that this is possible.

Lemma 6.2. Suppose $x^{(0)}(\alpha, t), \phi^{(0)}(\alpha, t)$ is a smooth, exact solution of the water wave equations (1.1), (1.2). Then there exist smooth functions $x^{(1)}(\alpha, t), \phi^{(1)}(\alpha, t)$ differing from $x^{(0)}(\alpha, t), \phi^{(0)}(\alpha, t)$ by $O\left(h^{3}\right)$, so that if $v^{(1)}$ is found from $x^{(1)}, \phi^{(1)}$ according to (6.1), (6.2), (1.25)-(1.30), then (6.3) holds for $x^{(1)}, \phi^{(1)}, v^{(1)}$ for any $p<4$. Also $w^{(1)}$ is within $O\left(h^{3}\right)$ of $\phi_{n}^{(1)}$ in $L_{h}^{2}$.

For the rest of this section we compare quantities such as $v^{(1)}$, found by the scheme from $x^{(1)}, \phi^{(1)}$ at each time, with the corresponding quantities in the computed solution. For simplicity we will drop the superscript (1) for the former. In contrast, we use tildes for the computed solution, determined by

$$
\tilde{x}_{t}=\tilde{v}, \quad \tilde{\phi}_{t}=\frac{1}{2}|\tilde{v}|^{2}-g \tilde{x}_{3}
$$

with $\tilde{v}$ found from $\tilde{x}, \tilde{\phi}$ as in (6.1), (6.2), (1.25)-(1.30). We write $\delta x$ for $\tilde{x}-x$, and similarly for other differences. Thus, e.g., $X_{1}=D_{h, 1} x, \tilde{X}_{1}=D_{h, 1} \tilde{x}, \delta X_{1}=\tilde{X}_{1}-X_{1}$. Then $\delta v=\tilde{v}-v$ is the error in the computed velocity due to $\delta x, \delta \phi$. Comparing (6.3) and (6.4), we have

$$
(\delta x)_{t}=\delta v+O\left(h^{p}\right), \quad(\delta \phi)_{t}=\frac{1}{2} \delta|v|^{2}-g \delta x_{3}+O\left(h^{p}\right) \quad \text { in } L_{h}^{2}, \quad p<4 .
$$

We will show that $\delta x, \delta \phi=O\left(h^{p-1 / 2}\right)$ in $L_{h}^{2}$. With $p$ near 4 , it will then follow from the triangle inequality that $\tilde{x}, \tilde{\phi}$ differ from the original exact solution in $L_{h}^{2}$ by $O\left(h^{3}\right)$, thereby completing the proof that the scheme converges.

Our main task now is to express the error $\delta v$ in computed velocity in terms of $\delta x, \delta \phi$. To do this we need to find the errors in such quantities as $n, \nabla_{h}^{T} \phi$, and $w$. For the latter we vary the integral equation which determines $w$.

Because we are proving that $\delta x, \delta \phi$ are almost $O\left(h^{7 / 2}\right)$ in $L_{h}^{2}$, we will assume a lower order bound on the errors,

$$
|\delta x|_{L_{h}^{2}} \leq h^{3}, \quad|\delta \phi|_{L_{h}^{2}} \leq h^{3}, \quad|\delta w|_{L_{h}^{2}} \leq h^{2}, \quad 0 \leq t \leq T .
$$

It follows that

$$
|\delta x|_{L_{h}^{\infty}} \leq h^{2}, \quad|\delta \phi|_{L_{h}^{\infty}} \leq h^{2}, \quad|\delta w|_{L_{h}^{\infty}} \leq h, \quad 0 \leq t \leq T .
$$

as well as $\left|\delta D_{h} x\right|_{L_{h}^{\infty}} \leq C_{0} h$, etc. This assumption is helpful in estimating nonlinear terms in the $\delta$ 's. It will be removed at the end of the argument. 
In order to make use of the gain of derivatives in certain operators related to $G_{h}^{\pi}$, seen in $\$ 5$ we will use the discrete Sobolev space $H_{h}^{-1}$ of grid functions with norm

$$
|f|_{H_{h}^{-1}}^{2}=\sum_{k \in I}\left(1+|k|^{2}\right)^{-1}|\ddot{f}(k)|^{2} h^{2} .
$$

We can write $f=E \cdot E^{-1} f$, so that $\left|E^{-1} f\right|_{L_{h}^{2}}=|f|_{H_{h}^{-1}}$, with $E$ as in (4.16). Thus if an operator $A$ is of order -1 on $L_{h}^{2}$, in the language of $\$ 5$, then $A$ is bounded from $H_{h}^{-1}$ to $L_{h}^{2}$, independent of $h$. In the next lemma we note some facts which will be used.

Lemma 6.3. We have $|f|_{H_{h}^{-1}} \leq|f|_{L_{h}^{2}}$ and $|f|_{L_{h}^{2}} \leq C h^{-1}|f|_{H_{h}^{-1}}$ for any $f$. The operators $D_{h, \ell}, D_{h, \ell}^{*}$ are bounded from $L_{h}^{2}$ to $H_{h}^{-1}$, uniformly in $h$. If $\psi$ is a grid function which either is the restriction of a smooth function or satisfies $|\psi|_{L_{h}^{\infty}} \leq C h$, then $|\psi f|_{H_{h}^{-1}} \leq C_{0}|f|_{H_{h}^{-1}}$, where $C_{0}$ depends on $\psi$ but not on $h$.

Proof. The first two statements follow from definitions. For the last, we represent elements of $H_{h}^{-1}$ as differences. Let $D_{h, \ell}^{+}$be the first-order forward difference in direction $\ell, \ell=1,2$. Since the Fourier symbol of $D_{h, \ell}^{+}$is bounded above and below by $\left|k_{\ell}\right|$ for $k \in I$, we can write any $f \in H_{h}^{-1}$ as $f=f_{0}+D_{h, 1}^{+} f_{1}+D_{h, 2}^{+} f_{2}$, where $f_{0}, f_{1}, f_{2}$ are bounded in $L_{h}^{2}$ by the $H_{h}^{-1}$ norm of $f$. Then, e.g., $\psi D_{h, 1}^{+} f_{1}=$ $D_{h, 1}^{+}\left(\psi f_{1}\right)-\left(D_{h, 1}^{+} \psi\right) f_{1}^{\sharp}$, where $f_{1}^{\sharp}$ is $f_{1}$ with a coordinate shift. The factor $D_{h, 1}^{+} \psi$ is uniformly bounded under either hypothesis on $\psi$, and it follows that $\psi D_{h, 1}^{+} f_{1}$ has the appropriate bound in $H_{h}^{-1}$, and in turn the same holds for $\psi f$.

Lemma 6.4. Assume (6.6). Then for the tangent vectors $X_{\ell}, \tilde{X}_{\ell}, \ell=1,2$, we have $\left|\tilde{X}_{\ell}-X_{\ell}\right|_{H_{h}^{-1}} \leq C|\tilde{x}-x|_{L_{h}^{2}}$. The same is true for the errors in $N,|N|$, n, and $X_{\ell}^{*}$.

Proof. The statement for $X_{\ell}$ is apparent from the definition of the norm. For the other quantities we use the following general fact: Suppose $u$ is a smooth function of $\alpha$ on the periodic square, possibly vector valued, and suppose $\tilde{u}_{j}$ is an approximation to $u\left(\alpha_{j}\right)$ such that $|\tilde{u}-u|_{H_{h}^{-1}} \leq C|\tilde{x}-x|_{L_{h}^{2}}$. Assume (6.6), and let $F$ be a smooth function on an open set covering the range of $u$. Then

$$
|F(\tilde{u})-F(u)|_{H_{h}^{-1}} \leq C^{\prime}|\tilde{x}-x|_{L_{h}^{2}} .
$$

The lemma follows from this fact, since the various quantities are functions of $X_{1}, X_{2}$.

To verify this claim, we first note that, for each $\alpha_{j}$,

$$
\left|\tilde{u}_{j}-u\left(\alpha_{j}\right)\right| \leq C h^{-1}|\delta u|_{L_{h}^{2}} \leq C h^{-2}|\delta u|_{H_{h}^{-1}} \leq C h^{-2} \cdot h^{3}=O(h)
$$

by hypothesis, so that $\tilde{u}$ is uniformly close to $u$. Next, with $u=u\left(\alpha_{j}\right)$, we write $F(\tilde{u})-F(u)=D F(u)(\delta u)+R(\tilde{u}, u)(\delta u)^{2}$, where $R$ is an average of the second derivative of $F$ between $u$ and $\tilde{u}$. The first term is bounded in $H_{h}^{-1}$ by $C|\delta u|_{H_{h}^{-1}}$ according to Lemma 6.3, since $D F(u)$ is a smooth factor. For the second term we estimate in $L_{h}^{2}$. The first factor is uniformly bounded, and for the rest we have

$$
\left|(\delta u)^{2}\right|_{L_{h}^{2}} \leq C|\delta u|_{L_{h}^{\infty}}|\delta u|_{L_{h}^{2}} \leq C h|\delta u|_{L_{h}^{2}} \leq C h \cdot h^{-1}|\delta u|_{H_{h}^{-1}}
$$

where we have used (6.10). Thus both terms are bounded by $|\delta u|_{H_{h}^{-1}} \leq C|\delta x|_{L_{h}^{2}}$, and (6.9) is proved. 
In order to simplify the expressions for errors, we will not be specific about terms contributing to $\delta v$ which are bounded in $L_{h}^{2}$ by $|\delta x|_{L_{h}^{2}},|\delta \phi|_{L_{h}^{2}}$, since they do not affect the numerical stability of the scheme. For this reason we use the generic notation $B(f)$ for a grid function which is bounded in $L_{h}^{2}$, uniformly in $h$, by $|f|_{L_{h}^{2}}$. Thus we write remainders such as $B(\delta x), B(\delta \phi)$.

In the next lemma we find the error in the unit normal, $\delta n=\tilde{n}-n$, due to $\delta x=\tilde{x}-x$, and also the error in the tangential gradient of an arbitrary function $\mu$, defined as in (6.1). Here and below we use the product rule (1.17) for $D_{h}$.

Lemma 6.5. Assuming (6.6), the error in $n$ due to $\delta x$ is

$$
\delta n=-\nabla_{h}^{T}(n \cdot \delta x)+B(\delta x) .
$$

Similarly, if $\mu$ is a smooth function of $\alpha$ and $\tilde{\mu}$ is an approximation on the grid, then

$$
\begin{aligned}
& \delta\left(\nabla_{h}^{T} \mu\right)=\nabla_{h}^{T}(\delta \mu)-\nabla_{h}^{T}\left(\delta x \cdot \nabla_{h}^{T} \mu\right)-\left(\delta n \cdot \nabla_{h}^{T} \mu\right) n+B(\delta x)+B(\delta \mu), \\
& =\nabla_{h}^{T}(\delta \mu)-\nabla_{h}^{T}\left(\delta x \cdot \nabla_{h}^{T} \mu\right)+\left(\nabla_{h}^{T}(n \cdot \delta x) \cdot \nabla_{h}^{T} \mu\right) n+B(\delta x)+B(\delta \mu) .
\end{aligned}
$$

Proof. Thinking of $n=X_{1} \times X_{2}$ as a pointwise function of $X_{k}=D_{h, k} x, k=1,2$, we introduce the infinitesimal change

$$
\dot{n}=\sum_{k=1,2}\left(\partial n / \partial X_{k}\right) \cdot \delta X_{k},
$$

where of course $\delta X_{k}=\tilde{X}_{k}-X_{k}$. Then

$$
\delta n\left(\alpha_{j}\right)=\dot{n}\left(\alpha_{j}\right)+O\left(\left|\delta X_{k}\left(\alpha_{j}\right)\right|^{2}\right) .
$$

Differentiating $n \cdot n=1$ shows $\dot{n} \cdot n=0$, i.e., $\dot{n}\left(\alpha_{j}\right)$ is tangential at $x\left(\alpha_{j}\right)$, and similarly $\dot{n} \cdot X_{k}=-n \cdot \delta X_{k}$. Then

$$
\dot{n}=\sum_{k=1,2}\left(\dot{n} \cdot X_{k}\right) X_{k}^{*}=-\sum_{k=1,2}\left(n \cdot \delta X_{k}\right) X_{k}^{*} .
$$

Now since $\delta X_{k}=D_{h, k}(\delta x)$, we can write $n \cdot \delta X_{k}=D_{h, k}(\delta x \cdot n)+B(\delta x)$, Then the above becomes $\dot{n}=-\nabla_{h}^{T}(\delta x \cdot n)+B(\delta x)$. We combine this with (6.16) to obtain the result (6.12); the remainder in (6.16) can be estimated in $L_{h}^{2}$ using (6.6) by

$$
\left|D_{h}(\delta x)\right|_{L_{h}^{\infty}}\left|D_{h}(\delta x)\right|_{L_{h}^{2}} \leq C h^{-1}\left|D_{h}(\delta x)\right|_{L_{h}^{2}}^{2} \leq C h^{-3}|\delta x|_{L_{h}^{2}}^{2} \leq C|\delta x|_{L_{h}^{2}} .
$$

Next we find a similar expression for $\delta X_{k}^{*}=\tilde{X}_{k}^{*}-X_{k}^{*}$. As before, let $\dot{X}_{k}^{*}$ be the infinitesimal change in $X_{k}^{*}$ due to $\delta x$. Again $\delta X_{k}^{*}-\dot{X}_{k}^{*}$ is quadratic in $D_{h}(\delta x)$, and it can be estimated as in (6.18). We have $\dot{X}_{k}^{*} \cdot X_{\ell}=-X_{k}^{*} \cdot \delta X_{\ell}$ and $\dot{X}_{k}^{*} \cdot n=-X_{k}^{*} \cdot \dot{n}$. Then

$$
\dot{X}_{k}^{*}=\sum_{\ell=1,2}\left(\dot{X}_{k}^{*} \cdot X_{\ell}\right) X_{\ell}^{*}+\left(\dot{X}_{k}^{*} \cdot n\right) n=-\sum_{\ell=1,2}\left(X_{k}^{*} \cdot \delta X_{\ell}\right) X_{\ell}^{*}-\left(X_{k}^{*} \cdot \dot{n}\right) n,
$$

and, after moving the $\ell$-derivative and replacing $\dot{n}$,

$$
\delta X_{k}^{*}=-\nabla_{h}^{T}\left(\delta x \cdot X_{k}^{*}\right)+\left(\nabla_{h}^{T}(n \cdot \delta x) \cdot X_{k}^{*}\right) n+B(\delta x)
$$

Finally,

$$
\delta\left(\nabla_{h}^{T} \mu\right)=\sum_{k=1,2} D_{h, k}(\delta \mu) X_{k}^{*}+\left(D_{h, k} \mu\right) \delta X_{k}^{*}+D_{h, k}(\delta \mu) \delta X_{k}^{*} .
$$

The last term can be estimated by $C|\delta \mu|_{L_{h}^{2}}$ using (6.6). Combining (6.20), (6.21) we then obtain (6.14), and ( $\underline{6.13})$ follows. 
In the next lemma we find an expression for $\delta v$ in terms of $\delta w$.

Lemma 6.6. With $v$ as in (6.2) and similarly for $\tilde{v}$, we have, assuming (6.6),

$$
\delta v=\nabla_{h}^{T}(\delta \phi-v \cdot \delta x)+(\delta w) n-\left(\delta n \cdot \nabla_{h}^{T} \phi\right) n+B(\delta x)+B(\delta \phi) .
$$

Proof. Varying (6.2), we have

$$
\delta v=\delta\left(\nabla_{h}^{T} \phi\right)+\delta(w n)=\delta\left(\nabla_{h}^{T} \phi\right)+(\delta w) n+w(\delta n)+(\delta w)(\delta n) .
$$

From (6.13) we have

$$
\delta\left(\nabla_{h}^{T} \phi\right)=\nabla_{h}^{T}(\delta \phi)-\nabla_{h}^{T}\left(\nabla_{h}^{T} \phi \cdot \delta x\right)-\left(\delta n \cdot \nabla_{h}^{T} \phi\right) n+B(\delta x)+B(\delta \phi),
$$

and from 6.12)

$$
w(\delta n)=-w \nabla_{h}^{T}(n \cdot \delta x)+B(\delta x)=-\nabla_{h}^{T}(w n \cdot \delta x)+B(\delta x) .
$$

For the last term in (6.23) we can estimate, using (6.6) and Lemma 6.4.

$$
|(\delta w)(\delta n)|_{L_{h}^{2}} \leq C|\delta w|_{L_{h}^{\infty}}|\delta n|_{L_{h}^{2}} \leq C h \cdot h^{-1}|\delta n|_{H_{h}^{-1}} \leq C|\delta x|_{L_{h}^{2}} .
$$

Now we substitute (6.24)-(6.26) into (6.23). We can combine terms in (6.24), (6.25) using (6.2) to obtain (6.22).

The calculation of $\delta v$ is now reduced to that of $\delta w$. To find $\delta w$, we subtract the discrete integral equation (1.25) for $w$ from that for $\tilde{w}$, and obtain an expression with variations in the discrete integrals entering the equation. We state the variations below; they are derived in $\S 7$. In the sums, $\nabla G_{h}^{\pi}=\nabla G_{h}^{\pi}\left(x_{j}-x_{\ell}\right)$, etc., while in the integrals $\nabla G_{h}^{\pi}=\nabla G_{h}^{\pi}\left(x_{j}-x\left(\alpha^{\prime}\right)\right)$.

$$
\begin{gathered}
\delta \sum_{\ell} \nabla G_{h}^{\pi} \cdot N_{\ell}\left[\left(n_{\ell} \cdot n_{j}\right) w_{\ell}-w_{j}\right] h^{2}=\sum_{\ell} \nabla G_{h}^{\pi} \cdot N_{\ell}\left[\left(n_{\ell} \cdot n_{j}\right) \delta w_{\ell}-\delta w_{j}\right] h^{2} \\
\quad+\left(\delta n_{j}\right) \cdot \int\left(\nabla G_{h}^{\pi} \cdot N\left(x\left(\alpha^{\prime}\right)\right) n\left(x\left(\alpha^{\prime}\right)\right) \phi_{n}\left(\alpha^{\prime}\right) d \alpha^{\prime}+h B(\delta w)+B(\delta x),\right.
\end{gathered}
$$

$$
\begin{aligned}
\delta \sum_{\ell} \nabla_{h}^{T} G_{h}^{\pi} \cdot n_{j} w_{\ell}\left|N_{\ell}\right| h^{2}=\sum_{\ell} \nabla_{h}^{T} G_{h}^{\pi} \cdot n_{j}\left(\delta w_{\ell}\right)\left|N_{\ell}\right| h^{2} \\
\quad+\sum_{\ell} \nabla_{h}^{T} G_{h}^{\pi} \cdot \nabla_{h}^{T}\left(w_{\ell} n_{\ell} \cdot \delta x_{\ell}\right)\left|N_{\ell}\right| h^{2} \\
+\left(\delta n_{j}\right) \cdot \int \nabla^{T} G_{h}^{\pi} \phi_{n}\left(\alpha^{\prime}\right)\left|N\left(\alpha^{\prime}\right)\right| d \alpha^{\prime}+h B(\delta w)+B(\delta x),
\end{aligned}
$$

$$
\begin{gathered}
\delta\left[n_{j} \cdot \sum_{\ell}\left[D_{h} G_{h}^{\pi}, X\right]_{\ell} \times\left(\nabla_{h}^{T} \phi_{\ell}-\nabla_{h}^{T} \phi_{j}\right) h^{2}-n_{j} \cdot \sum_{\ell} \nabla G_{h}^{\pi} \cdot N_{\ell}\left(\nabla_{h}^{T} \phi_{\ell}-\nabla_{h}^{T} \phi_{j}\right) h^{2}\right] \\
=\sum_{\ell} \nabla_{h}^{T} G_{h}^{\pi} \cdot \nabla_{h}^{T}\left(\delta \phi_{\ell}-\nabla_{h}^{T} \phi_{\ell} \cdot \delta x_{\ell}\right)\left|N_{\ell}\right| h^{2} \\
+\left(\delta n_{j}\right) \cdot \int \nabla G_{h}^{\pi} \times\left(N\left(\alpha^{\prime}\right) \times \nabla^{T} \phi\left(\alpha^{\prime}\right)\right) d \alpha^{\prime}+B(\delta x)+B(\delta \phi) .
\end{gathered}
$$

When we substitute these into the integral equation for $\delta w$, we can collect the terms. The integrals in (6.27) and (6.28) can be combined, and the second sum in (6.28) can be combined with the sum in (6.29) using (6.2). The first terms in (6.27) and (6.28) have the form $\mathcal{K}_{h}(\delta w)$, with $\mathcal{K}_{h}$ defined by (1.26), except for 
the correction. It can be checked that the error in the correction terms is of type $h B(\delta w)+B(\delta x)+B(\delta \phi)$; the operator $h P_{h} D_{h}^{*}$ is bounded from $H_{h}^{-1}$ to $L_{h}^{2}$ because of the high $k$ cut-off. Consequently we get

$$
\left(\frac{1}{2} I+\mathcal{K}_{h}\right)(\delta w)=\mathcal{L}(\delta \phi-v \cdot \delta x)+(\delta n) \cdot S+h B(\delta w)+B(\delta x)+B(\delta \phi),
$$

where the operator $\mathcal{L}$ and the function $S$ are defined by

$$
\begin{gathered}
(\mathcal{L} f)_{j}=\sum_{\ell} \nabla_{h}^{T} G_{h}^{\pi}\left(x_{j}-x_{\ell}\right) \cdot\left(\nabla_{h}^{T} f_{\ell}\right)\left|N_{\ell}\right| h^{2}, \\
S_{j}=\int \nabla G_{h}^{\pi} \times\left(N\left(\alpha^{\prime}\right) \times \nabla^{T} \phi\left(\alpha^{\prime}\right)\right) d \alpha^{\prime}-\int \nabla G_{h}^{\pi} \phi_{n}\left(\alpha^{\prime}\right)\left|N\left(\alpha^{\prime}\right)\right| d \alpha^{\prime} .
\end{gathered}
$$

Lemma 6.1 assures us that (6.30) can be solved for $\delta w$; the solvability is not affected by the small term $h B(\delta w)$. The following related lemma guarantees that $\tilde{w}$ can be found from its discrete integral equation.

Lemma 6.7. Under assumption (6.6) the operator $I+2 \tilde{\mathcal{K}}_{h}$, obtained by replacing $x$ with $\tilde{x}$ in (1.26), is invertible for $h$ sufficiently small, and the operator norm of the inverse is uniformly bounded. The discrete integral equation can be solved by the simple iteration (1.34).

The principal part of the operator $\mathcal{L}$ is of central importance in estimating the growth of numerical error. The following lemma identifies this principal part and its properties.

Lemma 6.8. The operator $\mathcal{L}$ on $L_{h}^{2}$ defined by (6.31) has the form $\mathcal{L}=\frac{1}{2} \Lambda+B_{0}$, where $B_{0}$ is bounded on $L_{h}^{2}$, uniformly in $h$, and $\Lambda$ is an operator with the following properties:

(i) $\Lambda$ is self-adjoint as an operator on $L_{h}^{2}, \Lambda f$ is real-valued for real $f$, and

$$
(\Lambda f, f)_{L_{h}^{2}} \geq c_{0}\left|E^{1 / 2} f\right|_{L_{h}^{2}}^{2}=c_{0} \sum_{k \in I}\left(1+|k|^{2}\right)^{1 / 2}|\ddot{f}(k)|^{2} .
$$

(ii) There are operators $B_{1}, \ldots, B_{5}$, bounded on $L_{h}^{2}$, uniformly in $h$, so that

$$
\begin{gathered}
\Lambda=B_{1} E, \quad \Lambda=E B_{2}, \\
\Lambda=E^{1 / 2} B_{3} E^{1 / 2}, \quad E=B_{4} \Lambda+B_{5} .
\end{gathered}
$$

Here $E$ is the absolute derivative defined in (4.16).

Proof. The result depends on the assumptions (1.14)-(1.16) for $D_{h}$, as well as the positivity of $-\hat{G}_{h}$. If we write out $\nabla_{h}^{T}$, we have

$$
\mathcal{L} f\left(\alpha_{j}\right)=-\sum_{\ell, \mu, \nu} D_{h, \mu} G_{h}^{\pi}\left(x_{j}-x_{\ell}\right)\left(D_{h, \nu} f_{\ell}\right)\left(X_{\mu \ell}^{*} \cdot X_{\nu \ell}^{*}\right)\left|N_{\ell}\right| h^{2}
$$

with $\mu, \nu=1,2$, or, after summing by parts and setting $b_{\ell}^{\mu \nu}=\left(X_{\mu \ell}^{*} \cdot X_{\nu \ell}^{*}\right)\left|N_{\ell}\right|=$ $\left(g^{\mu \nu}\right)_{\ell}\left|N_{\ell}\right|$,

$$
\mathcal{L} f\left(\alpha_{j}\right)=-\sum_{\ell, \mu, \nu} G_{h}^{\pi} D_{h, \mu}^{*}\left[b_{\ell}^{\mu \nu} D_{h, \nu} f_{\ell}\right] h^{2}=-\sum_{\ell, \mu, \nu} G_{h}^{\pi} b_{\ell}^{\mu \nu} D_{h, \mu}^{*} D_{h, \nu} f_{\ell} h^{2}+(B f)\left(\alpha_{j}\right) .
$$

To see that the remainder is bounded, we use (1.17) for $D_{h, \mu}^{*}$ and note that the operator with kernel $G_{h}^{\pi}$, times a smooth factor, is of order -1, according to Theorem 5.1. Next we replace $b_{\ell}^{\mu \nu}$ with $b_{j}^{\mu \nu}$; the difference is an operator applied to 
$D_{h, r}^{*} D_{h, \nu} f$ with the kernel $G_{h}^{\pi}$ times a function which vanishes at $\ell=j$. This operator has order -2 , by Theorem [5.1, and so the error is again bounded in $f$. We now have

$$
\mathcal{L} f\left(\alpha_{j}\right)=-\sum_{\mu, \nu} b_{j}^{\mu \nu}\left(A D_{h, \mu}^{*} D_{h, \nu} f\right)\left(\alpha_{j}\right)+(B f)\left(\alpha_{j}\right),
$$

where $A$ is the operator of Theorem 5.1, with kernel $G_{h}^{\pi}$. There we wrote $A$ as $A=-A^{(1)}-A^{(2)}$, where $A^{(2)}$ is of order -2 , and thus contributes a bounded term to $\mathcal{L}$, while $A^{(1)}$ is an operator of type (4.2) whose symbol $a_{1}(\alpha, k)$ is of class $S^{-1}$ with $a_{1}(\alpha, k) \geq c_{1}\left(1+|k|^{2}\right)^{-1 / 2}$ for $k \in I$. Thus $\mathcal{L}=\Lambda_{0}+B$, where $\Lambda_{0}$ is the operator of type (4.2) with symbol $\lambda_{0}$ of class $S^{1}$,

$$
\lambda_{0}(\alpha, k)=h^{-2} a_{1}(\alpha, k) \sum_{\mu, \nu} b^{\mu \nu}(\alpha) \bar{\sigma}\left(k_{\mu} h\right) \sigma\left(k_{\nu} h\right) .
$$

We have $\lambda_{0}(-k)=\bar{\lambda}_{0}(k)$, which implies that $\Lambda_{0}$ preserves real functions. Since the matrix $b^{\mu \nu}$ is positive definite and symmetric, and $h^{-1}\left|\sigma\left(k_{\mu} h\right)\right| \geq C\left|k_{\mu}\right|$ for $k \in I$, we have $\lambda_{0}(\alpha, k) \geq c_{2}|k|$ for $k \in I$. Thus Lemma 4.5 applies to $\Lambda_{0}$; the estimate (4.18) holds for $\Lambda_{0}$, and $\Lambda_{0}^{*}-\Lambda_{0}$ is bounded. Now we set $\Lambda=\Lambda_{0}^{*}+\Lambda_{0}+C_{0}$, where $C_{0}$ is a large enough constant. Then $\Lambda-2 \Lambda_{0}$ is bounded, and the properties of $\Lambda$ follow from Lemmas 4.44 .6

In view of this lemma, equation (6.30) reduces to

$$
\left(I+2 \mathcal{K}_{h}\right)(\delta w)=\Lambda(\delta \phi-v \cdot \delta x)+2(\delta n) \cdot S+h B(\delta w)+B(\delta x)+B(\delta \phi) .
$$

We now show that the term $\mathcal{K}_{h}(\delta w)$ is negligible. The two main terms on the right above can be written as $E f+B(\delta x)+B(\delta \phi)$ for some $f$ with $|f|_{L_{h}^{2}} \leq C\left(|\delta x|_{L_{h}^{2}}+\right.$ $\left.|\delta \phi|_{L_{h}^{2}}\right)$; this follows from (6.12) and (6.34). Then $\delta w$ is $\left(I+2 \mathcal{K}_{h}\right)^{-1} E f$ plus a remainder, and the main term in $\mathcal{K}_{h}(\delta w)$ is $\left(I+2 \mathcal{K}_{h}\right)^{-1} \mathcal{K}_{h} E f$. By Lemma 6.1 the last is bounded in $L_{h}^{2}$ norm by $\left|\mathcal{K}_{h} E f\right|_{L_{h}^{2}}$. Theorems 5.1 and 5.2 imply that the operator $\mathcal{K}_{h}$ is of order -1 ; for the second term in $\mathcal{K}_{h}$ we use the fact that $X_{k \ell}^{*} \cdot n_{j}=0$ when $\ell=j$. Thus $\left|\mathcal{K}_{h} E f\right|_{L_{h}^{2}} \leq C|f|_{L_{h}^{2}}$, and it follows that $\mathcal{K}_{h}(\delta w)=$ $h B(\delta w)+B(\delta x)+B(\delta \phi)$. We can now absorb $\mathcal{K}_{h}(\delta w)$ into the remainder in (6.40), and then invert $(I+h B)$ acting on $\delta w$, simplifying (6.40) to

$$
(\delta w)=\Lambda(\delta \phi-v \cdot \delta x)+2(\delta n) \cdot S+B(\delta x)+B(\delta \phi) .
$$

Next we substitute (6.41) into (6.22) to find a definitive expression for $\delta v$. After combining we have

$$
\delta v=\nabla_{h}^{T}(\delta \phi-v \cdot \delta x)+\Lambda(\phi-v \cdot \delta x) n+\delta n \cdot\left(2 S-\nabla^{T} \phi\right) n+B(\delta x)+B(\delta \phi) .
$$

(We have replaced $\nabla_{h}^{T} \phi$ with the exact $\nabla^{T} \phi$, at a cost of $h B(\delta n)=B(\delta x)$.) However, the third term above is negligible, as a consequence of potential theory: If $\Phi$ is a periodic harmonic function below the surface $x(\alpha)$, then

$$
2 \int \nabla^{T(x)} G^{\pi} \times\left(n(y) \times \nabla^{T} \Phi(y)\right) d S(y)-2 \int \nabla^{T(x)} G^{\pi} \frac{\partial \Phi}{\partial n(y)} d S(y)-\nabla^{T} \Phi(x)=0,
$$

where $G^{\pi}=G^{\pi}(x-y)$ and $\nabla^{T(x)}$ means the part of the gradient which is tangential at $x$. This follows from the same argument as for the integral equation (1.3), except that we take the limiting tangential derivative at the surface. If we replace $G^{\pi}$ by 
$G_{h}^{\pi}, \Phi$ by $\phi$, and $\partial \Phi / \partial n$ by $w$, the above holds with error $O(h)$, because of the regularization of $G$ and the modification in Lemma 6.2. The resulting expression almost matches $2 S-\nabla^{T} \phi$, except that the latter has $\nabla G^{\pi}$ rather than $\nabla^{T(x)} G^{\pi}$. But $\delta n$ is tangential except for a $B(\delta x)$ term; see (6.12). Therefore $\delta n \cdot\left(2 S-\nabla^{T} \phi\right)$ has the form $h B(\delta n)+B(\delta x)$ and thus $B(\delta x)$. In summary, we have

$$
\delta v=\nabla_{h}^{T}(\delta \phi-v \cdot \delta x)+\Lambda(\delta \phi-v \cdot \delta x) n+B(\delta x)+B(\delta \phi) .
$$

We want to replace the discrete approximation $v$ in (6.44) with $x_{t}$, the actual velocity of the modified exact solution. From (6.3) we see that $x_{t}-v$ is $O\left(h^{p}\right)$ in $L_{h}^{2}$ and thus $O\left(h^{p-1}\right)$ in $L_{h}^{\infty}$. The change in $\delta v$ is then $h^{p-2}(B(\delta x)+B(\delta \phi))$. Since $p>2$, this can be absorbed into the remainder. Thus we introduce

$$
u_{1}=\delta \phi-x_{t} \cdot \delta x
$$

and rewrite (6.44) as

$$
\delta v=\nabla_{h}^{T} u_{1}+\left(\Lambda u_{1}\right) n+B(\delta x)+B(\delta \phi) .
$$

We are now ready to estimate the growth in time of the errors in $\delta x, \delta \phi$. Because of the special structure of $\delta v$ in (6.46), we can use an argument similar to that in [5], [6]. We will replace $\delta x, \delta \phi$ by new state variables $u_{1}, u_{2}, u_{3}$, where $u_{1}$ is defined by 6.45 and

$$
u_{2}=\delta x \cdot n,
$$

so that, recalling (6.5), we have in $L_{h}^{2}$

$$
\begin{aligned}
u_{2, t}=(\delta x)_{t} \cdot n+B(\delta x)=\delta v \cdot n & +B(\delta x)+O\left(h^{p}\right) \\
& =\Lambda u_{1}+B(\delta x)+B(\delta \phi)+O\left(h^{p}\right) .
\end{aligned}
$$

We will choose $u_{3}$ to account for $(\delta x)^{T}$ while taking advantage of the similarity of the two main terms in (6.46). We will need to know that $B_{4}$ of Lemma 6.8, and later also $B_{3}$, have bounded time derivatives. This follows from their construction in $\$$ once we know that the $t$-derivative of the symbol $a_{1}(\alpha, k ; h)$ in Theorem 5.1 has bounds similar to those for $a_{1}$ itself, i.e., as a symbol of class $S^{-1}$. The essential point is that $G_{h}(J \alpha)$ depends on $t$ only through the Jacobian $J$, and its transform through the related matrix $M$ (see (5.11)); differentiation in $t$ does not change the degree of homogeneity in $\alpha$ or $k$, and the estimates for $G_{h}^{\pi}$ apply to $\partial G_{h}^{\pi} / \partial t$ as well.

To define $u_{3}$, we first write $\nabla_{h}^{T}=B_{6} \Lambda+B_{7}$, using (6.35), with $B_{6}, B_{7}$ bounded uniformly in $h, t$, determined by $B_{4}, B_{5}$. We now define

$$
u_{3}=(\delta x)^{T}-B_{6}(\delta x \cdot n) .
$$

Applying $\partial / \partial t$, using the boundedness of $B_{6, t}$, and dropping less important terms, we find that

$$
\begin{aligned}
u_{3, t}= & (\delta v)^{T}-B_{6}(\delta v \cdot n)+B(\delta x)+O\left(h^{p}\right) \\
& =\nabla_{h}^{T} u_{1}-B_{6}\left(\Lambda u_{1}\right)+B(\delta x)+O\left(h^{p}\right)=B(\delta x)+B(\delta \phi)+O\left(h^{p}\right),
\end{aligned}
$$

with no important terms remaining.

Next we derive the equation for $u_{1, t}$. From w6.5 obtain

$$
\delta \phi_{t}=\frac{1}{2} \delta(v \cdot v)-g \delta x_{3}+O\left(h^{p}\right)=v \cdot \delta v+\frac{1}{2}|\delta v|^{2}-g \delta x_{3}+O\left(h^{p}\right) .
$$

For the other term in $u_{1}$ we have

$$
\left(x_{t} \cdot \delta x\right)_{t}=x_{t} \cdot \delta v+x_{t t} \cdot \delta x+O\left(h^{p}\right) .
$$


When we subtract, we have a term $\left(v-x_{t}\right) \delta v$. From (6.5), $v-x_{t}=O\left(h^{p-1}\right)$ in $L_{h}^{\infty}$, and from (6.46) $\delta v$ has the form $h^{-1}(B(\delta x)+B(\delta \phi))$. Then this term is $h(B(\delta x)+B(\delta \phi))$, since $p>3$. Combining terms, we have

$$
u_{1, t}=-\left(x_{t t}+g e_{3}\right) \cdot \delta x+\frac{1}{2}|\delta v|^{2}+h B(\delta x)+h B(\delta \phi)+O\left(h^{p}\right) .
$$

For an exact solution of the Euler equations, $-\left(x_{t t}+g e_{3}\right)=\nabla p$, the pressure gradient. At the water surface, this is a normal vector, since $p=0$ there. Moreover, we saw in (1.35) that $-\nabla p \cdot n \geq c_{0}>0$ at the surface. We have modified the exact solution by $O\left(h^{3}\right)$, and thus in our case we have

$$
x_{t t}+g e_{3}=-c n+O\left(h^{3}\right), \quad c=c(\alpha, t) \geq c_{0}>0 .
$$

The first term in (6.53) is thus $-c \delta x \cdot n+h^{3} B(\delta x)=-c u_{2}+h^{3} B(\delta x)$. We need an estimate for the $(\delta v)^{2}$ term in (6.53). From (6.46) and (6.6) we have $|\delta v|_{L_{h}^{\infty}}=O(h)$, and also $|\delta v|_{L_{h}^{2}} \leq C\left|E u_{1}\right|_{L_{h}^{2}} \leq h^{-1 / 2}\left|E^{1 / 2} u_{1}\right|_{L_{h}^{2}}$, and combining these two facts, $\left|(\delta v)^{2}\right|_{L_{h}^{2}} \leq h^{1 / 2}\left|E^{1 / 2} u_{1}\right|_{L_{h}^{2}}$.

We can now estimate the growth of a norm of $\left(u_{1}, u_{2}, u_{3}\right)$. We define a norm so that the two principal terms will cancel in the growth estimates,

$$
U=\frac{1}{2}\left\{\left(\Lambda u_{1}, u_{1}\right)_{L_{h}^{2}}+\left|c^{1 / 2} u_{2}\right|_{L_{h}^{2}}^{2}+\left|c^{1 / 2} u_{3}\right|_{L_{h}^{2}}^{2}\right\} \equiv U_{1}+U_{2}+U_{3} .
$$

We have

$$
|\delta x|_{L_{h}^{2}}^{2}+|\delta \phi|_{L_{h}^{2}}^{2} \leq C U, \quad\left|E^{1 / 2} u_{1}\right|_{L_{h}^{2}}^{2} \leq C U
$$

and we can rewrite our equations as

$$
u_{1, t}=-c u_{2}+h^{1 / 2} r_{1}+h^{p} \tau_{1}, \quad u_{2, t}=\Lambda u_{1}+r_{2}+h^{p} \tau_{2}, \quad u_{3, t}=r_{3}+h^{p} \tau_{3},
$$

where the $r$ 's are bounded in $L_{h}^{2}$ by $U^{1 / 2}$, and the $\tau$ 's by a constant. Then

$$
\begin{aligned}
& U_{1, t}=\left(u_{1, t}, \Lambda u_{1}\right)+\frac{1}{2}\left(\Lambda_{t} u_{1}, u_{1}\right) \\
& \quad=-\left(c u_{2}, \Lambda u_{1}\right)+h^{1 / 2}\left(r_{1}, \Lambda u_{1}\right)+\frac{1}{2}\left(B_{3, t} E^{1 / 2} u_{1}, E^{1 / 2} u_{1}\right)+h^{p}\left(\tau_{1}, \Lambda u_{1}\right) .
\end{aligned}
$$

The second term can be estimated by

$$
C h^{1 / 2}\left|r_{1}\right|_{L_{h}^{2}}\left|E u_{1}\right|_{L_{h}^{2}} \leq C h^{1 / 2}\left|r_{1}\right|_{L_{h}^{2}} h^{-1 / 2}\left|E^{1 / 2} u_{1}\right|_{L_{h}^{2}} \leq C U
$$

and the third term by $C\left|E^{1 / 2} u_{1}\right|_{L_{h}^{2}}^{2} \leq C U$. Recalling that $\Lambda=B_{1} E$, we estimate the last term by

$$
C h^{p}\left|\Lambda u_{1}\right|_{L_{h}^{2}} \leq C h^{p}\left|E u_{1}\right|_{L_{h}^{2}} \leq C h^{p-1 / 2}\left|E^{1 / 2} u_{1}\right|_{L_{h}^{2}} \leq C h^{p-1 / 2} U^{1 / 2},
$$

and in summary

$$
U_{1, t}=\left(u_{1, t}, \Lambda u_{1}\right)+R_{1}, \quad\left|R_{1}\right| \leq C\left(U+h^{p-1 / 2} U^{1 / 2}\right) .
$$

For $U_{2}$ we have

$$
U_{2, t}=\left(c u_{2, t}, u_{2}\right)+\frac{1}{2}\left(c_{t} u_{2}, u_{2}\right)=\left(c \Lambda u_{1}, u_{2}\right)+R_{2}
$$

with $\left|R_{2}\right|$ bounded by $C\left(U+h^{p} U^{1 / 2}\right)$, and similarly $U_{3, t}=R_{3}$. When we add, the two main terms in (6.61), (6.62) cancel, and we have $U_{t} \leq C_{1}\left(U+h^{2 p-1}\right)$. Then $U(t) \leq C_{2} h^{2 p-1}$ for $0 \leq t \leq T$, and from (6.56)

$$
|\delta x|_{L_{h}^{2}}+|\delta \phi|_{L_{h}^{2}} \leq C_{3} h^{p-1 / 2}, \quad 0 \leq t \leq T .
$$


It follows from (6.46) and Lemma 6.6 that

$$
|\delta v|_{L_{h}^{2}},|\delta w|_{L_{h}^{2}} \leq C_{4} h^{p-3 / 2}, \quad 0 \leq t \leq T .
$$

We can now remove the assumption (6.6) used to derive (6.63), (6.64). We can take $p$ near 4 , so that $p-1 / 2$ is almost $7 / 2$. For $h$ small enough, (6.63), (6.64) imply that the $L_{h}^{2}$ norms of $\delta x, \delta \phi, h \delta w$ remain below $h^{3} / 2$ as long as they are less than $h^{3}$, and therefore they never reach $h^{3}$. Thus for small $h$ (6.63), (6.64) hold without qualification, and in view of Lemma 6.2 the computed solution stays within $O\left(h^{3}\right)$ of the actual solution in $L_{h}^{2}$.

\section{Proofs of LemMas}

Here we present the proofs of Lemma 6.1 Lemma 6.2, estimates 6.27)-(6.29), and Lemma 6.7 .

Proof of Lemma 6.1 The invertibility of $I+2 \mathcal{K}_{h}$ will follow from that of the operator $I+2 \mathcal{K}$ of which it is an approximation, where $\mathcal{K}$ is the operator acting on periodic functions of $\alpha$,

$$
\mathcal{K} f(\alpha)=\int \nabla G^{\pi}\left(x(\alpha)-x\left(\alpha^{\prime}\right)\right) \cdot n(x(\alpha)) f\left(\alpha^{\prime}\right)\left|N\left(\alpha^{\prime}\right)\right| d \alpha^{\prime} .
$$

Thus $\mathcal{K} f$ is the adjoint double layer potential due to $f$. With time $t$ fixed for the moment, the operator $\mathcal{K}$ is compact, as an operator on $L^{2}$ of the periodic square, so that its nonzero spectrum consists only of eigenvalues. Classical arguments show that the operator $2 \mathcal{K}$ and its adjoint $2 \mathcal{K}^{*}$ have spectral radius strictly less than 1 ; that is, if $\lambda$ is an eigenvalue of $2 \mathcal{K}$, then $|\lambda| \leq \rho(2 \mathcal{K})<1$. This fact depends on the periodic geometry; for bounded domains there is an eigenvalue with $|\lambda|=1$. A proof that $\rho(2 \mathcal{K})=\rho\left(2 \mathcal{K}^{*}\right)<1$ was given in [3] for double layer potentials in periodic regions in two dimensions; essentially the same proof applies to the present case of 3-D regions with doubly periodic boundaries.

Now let $\mathcal{K}(t)$ be the operator at time $t$ for $0 \leq t \leq T$. We argue that for some constants $\varepsilon>0$ and $C_{0}>0$, and for all complex $\lambda$ with $|\lambda| \geq 1-\varepsilon$, and all $t$ with $0 \leq t \leq T$, the operator $\lambda I-2 \mathcal{K}(t)$ is invertible, and $\left\|(\lambda I-2 \mathcal{K}(t))^{-1}\right\| \leq C_{0}$. First, since $\rho(2 \mathcal{K}(t))<1$, then also $\rho(2 \mathcal{K}(t)+A)<1$ if $A$ is small enough in norm as an operator on $L^{2}$; this is shown in [10, Theorem 1.37. It can be checked that $\mathcal{K}(t)$ depends continuously on $t$, as an operator on $L^{2}$, using the smoothness assumptions. Thus the above statement tells us that for each $t_{0}$ there is a $t$-interval near $t_{0}$ such that $\rho(2 \mathcal{K}(t))$ is bounded away from 1 on the interval. Since $[0, T]$ is a compact set, the same is true for this entire interval, i.e., there is some $\varepsilon>0$ so that $\rho(2 \mathcal{K}(t))<1-\varepsilon$ for each $t \in[0, T]$. This shows that $\lambda I-2 \mathcal{K}(t)$ is invertible for each $t$ and $\lambda$ with $|\lambda| \geq 1-\varepsilon$. The inverse depends continuously on $t, \lambda$, and so a uniform bound for the norm follows for $\lambda$ in a bounded set by compactness. Finally, we have a uniform bound for the norm of $\mathcal{K}(t)$, and from this we can easily obtain a bound for the inverse for large $\lambda$. This verifies the assertion.

The above fact allows us to show that operators close to $\mathcal{K}(t)$ have similar properties. Suppose $A$ is any operator on $L^{2}$ with $\|A\|<\left(2 C_{0}\right)^{-1}$, where $C_{0}$ is as above. Then for any $t$ and any $\lambda$ with $|\lambda| \geq 1-\varepsilon$, we have $\lambda I-(2 \mathcal{K}-A)=$ $(\lambda I-2 \mathcal{K})\left[I+(\lambda I-2 \mathcal{K})^{-1} A\right]$. By the statement above, each of the last two factors is invertible, and the inverse has norm bounded by $2 C_{0}$. Thus we have shown that $\rho(2 \mathcal{K}(t)-A)<1-\varepsilon$, and in particular $\left\|(I+2 \mathcal{K}(t)-A)^{-1}\right\| \leq 2 C_{0}$. To complete 
the proof of the lemma, it will be sufficient to show that $\mathcal{K}_{h}$ can be regarded as a small perturbation of $\mathcal{K}$ when $h$ is small.

We first treat the operator $\mathcal{K}_{h}^{0}$, which is $\mathcal{K}_{h}$ with the two terms with $w_{j}$ omitted. To show that $I+2 \mathcal{K}_{h}^{0}$ is invertible on $L_{h}^{2}$, we identify it with an operator on $L^{2}\left([-\pi, \pi)^{2}\right)$; i.e., we extend from the discrete variable $\alpha_{j} \in I h$ to $\alpha \in[-\pi, \pi)^{2}$. We partition the square by defining $B_{j}=\left\{\alpha \in[-\pi, \pi)^{2}: 0 \leq \alpha_{\nu}-\left(\alpha_{j}\right)_{\nu}<h, \nu=1,2\right\}$ for $j \in I$. If we write $\mathcal{K}_{h}^{0}$ in the form

$$
\mathcal{K}_{h}^{0} f\left(\alpha_{j}\right)=\sum_{\ell \in I} K_{h}\left(\alpha_{j}, \alpha_{\ell}\right) f\left(\alpha_{\ell}\right) h^{2},
$$

we can extend the kernel by defining $K_{h}\left(\alpha, \alpha^{\prime}\right)=K_{h}\left(\alpha_{j}, \alpha_{\ell}\right)$ for $\alpha \in B_{j}, \alpha^{\prime} \in B_{\ell}$. This kernel then gives an integral operator on $L^{2}\left([-\pi, \pi)^{2}\right)$. Now if $f$ is a function on $I h$, we can extend it to a piecewise constant function on $[-\pi, \pi)^{2}$ by defining $f(\alpha)=f\left(\alpha_{\ell}\right)$ for $\alpha \in B_{\ell}$. The integral operator applied to this extended $f$ produces a piecewise constant function with values given by (17.2). The $L^{2}$ norms of the extended $f$ and its image match the $L_{h}^{2}$ norms of the discrete functions. (Cf. [6], Lemma 5 ; 7], p. 16.) Thus if we show that the extended operator $I+2 \mathcal{K}_{h}^{0}$ has an inverse, bounded independent of $h$, then the same will be true for the discrete operator.

In view of the above remarks, it will be sufficient to show that the discrete operator $\mathcal{K}_{h}^{0}$, when extended, is close to the corresponding continuous version. The two terms of $\mathcal{K}_{h}^{0}$ have the form

$$
\begin{gathered}
J_{1} f\left(\alpha_{j}\right)=\sum_{\ell \in I} N_{\ell} \cdot \nabla G_{h}^{\pi}\left(x_{j}-x_{\ell}\right) \mu_{1}\left(\alpha_{j}, \alpha_{\ell}\right) f\left(\alpha_{\ell}\right) h^{2}, \\
J_{2} f\left(\alpha_{j}\right)=\sum_{\ell \in I} D_{h} G_{h}^{\pi}\left(x_{j}-x_{\ell}\right) \mu_{2}\left(\alpha_{j}, \alpha_{\ell}\right) f\left(\alpha_{\ell}\right) h^{2},
\end{gathered}
$$

where $\mu_{1}, \mu_{2}$ are smooth, $\mu_{2}$ is zero on the diagonal, $D_{h}$ is the discrete derivative with respect to $\alpha_{\ell}$, and $N_{\ell}$ is computed from $x(\alpha)$ using $D_{h}$. Thus, in the first case, we compare $J_{1}$ extended to $L^{2}$ with

$$
\int N\left(\alpha^{\prime}\right) \cdot \nabla G_{h}^{\pi}\left(x(\alpha)-x\left(\alpha^{\prime}\right)\right) \mu_{1}\left(\alpha, \alpha^{\prime}\right) f\left(\alpha^{\prime}\right) d \alpha^{\prime} .
$$

(We have already seen in Theorem 2.3 that the change in the integral due to regularizing $G$ to $G_{h}$ is $O\left(h^{3}\right)$.) The error to be accounted for is that from replacing $x(\alpha)$ by $x_{j}, x\left(\alpha^{\prime}\right)$ by $x_{\ell}$, and $N\left(\alpha^{\prime}\right)$ by $N_{\ell}$ in the kernel when $\alpha \in B_{j}, \alpha^{\prime} \in B_{\ell}$. We use an estimate for the first derivative of the kernel. We consider $G_{h}$ instead of $G_{h}^{\pi}$, since the remainder is smooth. Since $G_{h}$ is radial, we have

$$
N\left(\alpha^{\prime}\right) \cdot \nabla G_{h}\left(x(\alpha)-x\left(\alpha^{\prime}\right)\right)=\left|x(\alpha)-x\left(\alpha^{\prime}\right)\right|^{-1} \frac{\partial G_{h}}{\partial r}\left[N\left(\alpha^{\prime}\right) \cdot\left(x(\alpha)-x\left(\alpha^{\prime}\right)\right] .\right.
$$

The last factor is $O\left(\left|\alpha-\alpha^{\prime}\right|^{2}\right)$ for $\alpha$ near $\alpha^{\prime}$, since $x(\alpha)-x\left(\alpha^{\prime}\right)$ is almost tangent. From the estimates (2.4) for $G_{h}$, we have $\left|(\partial / \partial r)^{m} G_{h}\right| \leq C\left|\alpha-\alpha^{\prime}\right|^{-m-1}$ for $\left|\alpha-\alpha^{\prime}\right| \geq h$ and $O\left(h^{-m-1}\right)$ otherwise, where $m=1$ or 2 . Combining these facts, we find for the kernel $K\left(\alpha, \alpha^{\prime}\right)$ of (7.5) that $\left|D K\left(\alpha, \alpha^{\prime}\right)\right| \leq C\left|\alpha-\alpha^{\prime}\right|^{-2}$ for $\left|\alpha-\alpha^{\prime}\right| \geq h$ and $O\left(h^{-2}\right)$ otherwise; here $D$ denotes a first derivative in $\alpha$ or $\alpha^{\prime}$. Thus the error in discretizing the kernel is of order $h \cdot h^{-2}$ for $\alpha, \alpha^{\prime}$ close, and order $h \cdot\left|\alpha-\alpha^{\prime}\right|^{-2}$ when they are at least $O(h)$ apart. We can estimate the norm of the operator resulting from this error just as in the last part of the proof of Theorem [5.1 the 
norm is bounded by $C h|\log h|$. Replacing $N\left(\alpha_{\ell}\right)$ with $N_{\ell}$ leads to a smaller error, since they differ by $O\left(h^{3}\right)$.

We argue similarly for the second operator $J_{2}$. We need to compare the kernel $\mu_{2} D G_{h}^{\pi}\left(x(\alpha)-x\left(\alpha^{\prime}\right)\right)$ with discrete values of $\mu_{2} D_{h} G_{h}^{\pi}$. (Again the change in the integral from regularizing $G$ to $G_{h}$ is $O\left(h^{3}\right)$.) Assuming first that $D_{h}$ is a difference operator, the error in replacing $D G_{h}^{\pi}\left(x_{j}-x_{\ell}\right)$ with $D_{h} G_{h}^{\pi}\left(x_{j}-x_{\ell}\right)$ can be estimated by writing $\left(D_{h}-D\right) G_{h}^{\pi}$ as an integral of $D^{2} G_{h}^{\pi}$. This is controlled by a bound for $h \cdot D^{2} G_{h}^{\pi}$ on an $O(h)$ neighborhood of $x_{j}-x_{\ell}$. Since $\mu_{2}\left(\alpha_{j}, \alpha_{\ell}\right)=0$, we find that the contribution to the error in the kernel is then of order $h \cdot\left|\alpha_{j}-\alpha_{\ell}\right|^{-2}$ when $\alpha_{j}, \alpha_{\ell}$ are apart, and of order $h^{-1}$ when they are close. Just as before, this estimate leads to an error in the operator with norm of order $h|\log h|$. Next we assess the error in discretizing $\mu_{2} D G_{h}^{\pi}$. The error in the kernel has terms of the form $h \cdot \mu_{2} D^{2} G_{h}^{\pi}$ and $h \cdot D G_{h}^{\pi}$. For either of these we obtain the same estimates as before, using again the fact that $\mu_{2}=0$ on the diagonal. This completes the treatment of $J_{2}$ if $D_{h}$ is a difference operator. If not, we can reduce to the special case by comparing the discrete $\mu_{2} D_{h} G_{h}^{\pi}$ with one where $D_{h}$ is replaced by a difference operator. For this we note that $D_{h}^{(1)}-D_{h}^{(2)}$ has the form $h B E^{2}$ for some bounded $B$, and by Theorem 5.2 and (5.5), the two operators differ by $O(h)$.

In summary, we have shown that the error in discretizing each operator $J_{1}, J_{2}$ is $O(h|\log h|)$, and thus $\mathcal{K}_{h}^{0}-\mathcal{K}$ is of the same order. Finally we consider $\mathcal{K}_{h}-\mathcal{K}_{h}^{0}$. Each of the two terms multiplies $w_{j}$ by a factor. For the correction term this factor is $O(h)$; for the other term it is a sum which is within $O(h)$ of the first integral in (1.5), which is zero. Thus $\mathcal{K}_{h}-\mathcal{K}_{h}^{0}$ is $O(h)$, and therefore $\mathcal{K}_{h}-\mathcal{K}$ is $O(h|\log h|)$, as operators on $L_{h}^{2}$.

Proof of Lemma 6.2. The exact equations for $x, \phi$, as functions of $\alpha, t$, have the form $x_{t}=v, \phi_{t}=|v|^{2} / 2-g x_{3}$, with $v$ determined by $v=\nabla^{T} \phi+w n$, and with $\left(\frac{1}{2} I+\mathcal{K}(x)\right) w=f(x, \phi)$. We think of this system in the form $z_{t}=F(z)$, where $z=(x, \phi)$ and $F$ is a nonlocal mapping. Let $H^{s}$ be the Sobolev space of scalar-valued periodic functions of $\alpha$ with $s$ derivatives in $L^{2}, X^{s}$ the space of such vector-valued functions, and $Z^{s}$ the space of pairs $x \in X^{s}, \phi \in Y^{s}$. We choose $s_{0}>1$ and check that $F$ is $C^{2}$ from $Z^{s_{1}}$ to $Z^{s_{0}}$ when $s_{1}$ is large enough relative to $s_{0}$. First, the single and double layer potentials define bounded operators from $H^{s_{0}}$ to $H^{s_{0}+1}$, and they are at least $C^{2}$ as functions of $x \in X^{s_{1}}$; the $x$-derivatives do not change the order of the singularity. Then $f: Z^{s_{1}} \rightarrow H^{s_{0}}$ is $C^{2}$. Since $I+2 \mathcal{K}$ is a Fredholm operator on $H^{s_{0}}$ and is $1-1$, it is invertible, and the inverse is a $C^{2}$ function of $x \in X^{s_{1}}$. It now follows easily that $F: Z^{s_{1}} \rightarrow Z^{s_{0}}$ is $C^{2}$.

We need to use $D F\left(z^{0}\right)$, the derivative of $F$ at the exact, smooth solution $z^{0}$. We presume that $z^{0}$ exists in $Z^{s_{2}}$ for some $s_{2}$ larger than $s_{1}$. Finding $D F\left(z^{0}\right)$ amounts to linearizing the equations about $z^{0}$, and this can be done as for the discrete equations in $\S 6$ but more easily. Writing the infinitesimal variation in $z$ in the $u$ variables of (6.45), we have $D F\left(z^{0}\right) u=\left(-c u_{2}, \Lambda u_{1}, 0\right)+\left(0, B_{2} u, B_{3} u\right)$, where $\Lambda$ is a positive pseudodifferential operator of order 1 and $B_{2}, B_{3}$ are bounded in $H^{s_{1}}$ by $u_{2}, u_{3} \in H^{s_{1}}$. For solutions of $u_{t}=D F\left(z^{0}\right) u$, we can estimate as in 96 but in high norms. Let $U_{m}=\left(\Lambda D^{m} u_{1}, D^{m} u_{1}\right)_{L^{2}}+\left|D^{m}\left(c^{1 / 2} u_{2}\right)\right|_{L^{2}}^{2}+\left|D^{m}\left(c^{1 / 2} u_{3}\right)\right|_{L^{2}}^{2}$ where $m$ is a multi-index for the $\alpha$-derivative $D$, and let $U=\Sigma U_{m},|m| \leq s_{1}$. Then $U_{t} \leq C_{1} U$, and thus $U(t) \leq C_{2} U(0)$ for $0 \leq t \leq T$. (Cf. [5], p. 1282.)

The discrete equations have a similar structure $z_{h, t}=F_{h}\left(z_{h}\right)$, with $v_{h}$ determined by $w_{h}$ satisfying $\left(\frac{1}{2} I+\mathcal{K}_{h}\left(x_{h}\right)\right) w_{h}=f_{h}\left(z_{h}\right)$. The consistency argument shows 
that for any $z=(x, \phi) \in Z^{s_{1}}$ we have $\left(\frac{1}{2} I+\mathcal{K}_{h}(x)\right) \phi_{n}=f_{h}(z)+h^{3} r_{31}(z)+$ $r_{41}(z, h)$, where $r_{31}(z) \in H^{s_{0}}$, depending smoothly on $z \in Z^{s_{1}}$, and $r_{41}$ is uniformly $O\left(h^{4}\right)$. To invert, we use the fact that $\left(\frac{1}{2} I+\mathcal{K}_{h}(x)\right)^{-1}$ differs as an operator on $L^{2}$ from $\left(\frac{1}{2} I+\mathcal{K}(x)\right)^{-1}$ by $O(h|\log h|)$, as shown in the proof of Lemma 6.1. Thus we can write $\phi_{n}=\left(\frac{1}{2} I+\mathcal{K}_{h}(x)\right)^{-1} f_{h}(z)+h^{3} r_{32}(z)+r_{42}(z, h)$, where $r_{32}(z)=$ $\left(\frac{1}{2} I+\mathcal{K}(x)\right)^{-1} r_{31}(z)$ depends smoothly in $H^{s_{0}}$ on $z$ as above, and $r_{42}$ is bounded in $L_{h}^{2}$ by $C h^{4}|\log h|$. (The $\log h$ can be removed by a more careful argument.) Similarly, for any $z \in Z^{s_{1}}$, we get $F(z)-F_{h}(z)=h^{3} r_{3}(z)+r_{4}(z, h)$, where $r_{3}$ : $Z^{s_{1}} \rightarrow Z^{s_{0}}$ is smooth and $r_{4}(z, h)$ is $O\left(h^{4}|\log h|\right)$ in $L_{h}^{2}$. We can also assume that $r_{3}\left(z^{0}\right) \in Z^{s_{1}}$, since $z^{0} \in Z^{s_{2}}$.

Now we can combine the above to modify $z^{0}$. First, we can solve the linear equation $\eta_{t}=D F\left(z^{0}\right) \eta-r_{3}\left(z^{0}\right), \eta=0$ at $t=0$, with $\eta \in Z^{s_{1}}$; this can be done in a standard way from the linear estimate above. Now set $z^{1}=z^{0}+h^{3} \eta$ in $Z^{s_{1}}$. Then $F\left(z^{1}\right)=F\left(z^{0}\right)+h^{3} D F\left(z^{0}\right) \eta+O\left(h^{6}\right)$ in $Z^{s_{0}}$, since $F$ is $C^{2}$. We check that $z^{1}$ has the desired property. We have $z_{t}^{1}=F\left(z^{0}\right)+h^{3} D F\left(z^{0}\right) \eta-h^{3} r_{3}\left(z^{0}\right)=$ $F\left(z^{1}\right)-h^{3} r_{3}\left(z^{0}\right)+O\left(h^{6}\right)=F_{h}\left(z^{1}\right)-h^{3} r_{3}\left(z^{0}\right)+O\left(h^{6}\right)+h^{3} r_{3}\left(z^{1}\right)+r_{4}\left(z^{1}, h\right)$. Now since $z^{1}-z^{0}=O\left(h^{3}\right)$ in $Z^{s_{1}}$, we have $r_{3}\left(z^{1}\right)-r_{3}\left(z^{0}\right)=O\left(h^{3}\right)$ in $Z^{s_{0}}$. Thus the $O\left(h^{3}\right)$ part cancels, and and we conclude that $z_{t}^{1}=F_{h}\left(z^{1}\right)+O\left(h^{4}|\log h|\right)$ in $L_{h}^{2}$. The two components of this equation give (6.3) for $p<4$. The last assertion follows from combining this with (6.2).

Proof of (6.27). We need to assess the difference between

$$
\sum \nabla G_{h}^{\pi}\left(\tilde{x}_{j}-\tilde{x}_{\ell}\right) \cdot \tilde{N}_{\ell}\left[\left(\tilde{n}_{\ell} \cdot \tilde{n}_{j}\right) \tilde{w}_{\ell}-\tilde{w}_{j}\right] h^{2}
$$

and the same where the computed tilde quantities are replaced by the quantities without tildes. To do this we will consider various separate differences by adding and subtracting. (All sums are over $\ell \in I$.) We note first that under the hypothesis (6.6) we have, using Lemmas 6.3 and 6.4

$$
|\delta n|_{L_{h}^{\infty}} \leq C h^{-1}|\delta n|_{L_{h}^{2}} \leq C h^{-2}|\delta n|_{H_{h}^{-1}} \leq C h^{-2}|\delta x|_{L_{h}^{2}} \leq C h^{-2} h^{3}=C h,
$$

and similarly for $w, N$.

We begin with the sum

$$
\sum \nabla G_{h}^{\pi}\left(x_{j}-x_{\ell}\right) \cdot \tilde{N}_{\ell}\left[\left(\tilde{n}_{\ell} \cdot \tilde{n}_{j}\right) \tilde{w}_{\ell}-\tilde{w}_{j}\right] h^{2}
$$

and consider several differences which, when added to the above, will result in the corresponding sum with no tildes. The first such difference will be the replacement of $\tilde{N}_{\ell}$ by $N_{\ell}$. We split this into two terms,

$$
\begin{gathered}
\sum \nabla G_{h}^{\pi}\left(x_{j}-x_{\ell}\right) \cdot\left(\delta N_{\ell}\right)\left[\left(n_{\ell} \cdot n_{j}\right) w_{\ell}-w_{j}\right] h^{2}, \\
\sum \nabla G_{h}^{\pi}\left(x_{j}-x_{\ell}\right) \cdot\left(\delta N_{\ell}\right)\left[\delta\left(\left(n_{\ell} \cdot n_{j}\right) w_{\ell}\right)-\delta w_{j}\right] h^{2} .
\end{gathered}
$$

We view (7.10) as the action on $\delta N$ of the kernel $\nabla G_{h}^{\pi}$ times $\left(n_{\ell} \cdot n_{j}\right) w_{\ell}-w_{j}$. The latter factor is the restriction of a smooth function vanishing on the diagonal, except for a small error, according to the last statement in Lemma 6.2. We ignore the error, since it can be handled as below for (7.11). By Theorem 5.1 the resulting operator has order -1 on $L_{h}^{2}$, and according to the remark before Lemma 6.3 it is bounded from $H_{h}^{-1}$ to $L_{h}^{2}$. Thus the sum (7.10) is bounded in $L_{h}^{2}$ by $C|\delta N|_{H_{h}^{-1}} \leq C|\delta x|_{L_{h}^{2}}$. Now for (7.11), the quantity in brackets is uniformly $O(h)$, according to (7.8) and the analogue for $w$. We can use the boundedness of $\nabla G_{h}^{\pi}$ on $L_{h}^{2}$, again from Theorem 
[5.1, to estimate this term by $C h|\delta N|_{L_{h}^{2}} \leq C|\delta N|_{H_{h}^{-1}} \leq C|\delta x|_{L_{h}^{2}}$. Next, to replace $\tilde{n}_{\ell}$ by $n_{\ell}$, we consider the difference

$$
\sum \nabla G_{h}^{\pi}\left(x_{j}-x_{\ell}\right) \cdot N_{\ell}\left[\tilde{w}_{\ell}\left(\delta n_{\ell}\right)\right] \cdot \tilde{n}_{j} h^{2} .
$$

Now the operator $\nabla G_{h}^{\pi} \cdot N\left(\alpha_{\ell}\right)$ is of order -1 by Theorem 5.1 where $N\left(\alpha_{\ell}\right)$ is the exact normal. Since $N_{\ell}-N\left(\alpha_{\ell}\right)=O\left(h^{3}\right)$ and $\nabla G_{h}^{\pi}$ has order 0 , it follows that $\nabla G_{h}^{\pi} \cdot N_{\ell}$ is also of order -1 . Using this fact and Lemmas 6.3 and 6.4, we can estimate (7.12) by $C|\tilde{w}(\delta n)|_{H_{h}^{-1}} \leq C|\delta n|_{H_{h}^{-1}} \leq C|\delta x|_{L_{h}^{2}}$.

We have now replaced (7.9) with

$$
\sum \nabla G_{h}^{\pi}\left(x_{j}-x_{\ell}\right) \cdot N_{\ell}\left[\left(n_{\ell} \cdot \tilde{n}_{j}\right) \tilde{w}_{\ell}-\tilde{w}_{j}\right] h^{2} .
$$

If we replace $\tilde{w}_{\ell}, \tilde{w}_{j}$ with $w_{\ell}, w_{j}$, the resulting difference is the first term on the right in (6.27) plus

$$
\left(\delta n_{j}\right) \cdot \sum \nabla G_{h}^{\pi}\left(x_{j}-x_{\ell}\right) \cdot N_{\ell} n_{\ell}\left(\delta w_{\ell}\right) h^{2} .
$$

Since $\delta n_{j}$ is uniformly $O(h)$, the last is bounded in $L_{h}^{2}$ by $C h|\delta w|_{L_{h}^{2}}$. Then if we replace $\tilde{n}_{j}$ by $n_{j}$, the difference is

$$
\left(\delta n_{j}\right) \cdot \sum w_{\ell} n_{\ell} \nabla G_{h}^{\pi}\left(x_{j}-x_{\ell}\right) \cdot N_{\ell} h^{2} .
$$

In the sum above, we can replace $w_{\ell}$ with $\phi_{n}\left(\alpha_{\ell}\right)$, etc., using Lemma 6.2 with small error. The resulting sum is then within $O(h)$ of the integral term in 6.27), according to Theorem 3.7 The term from the quadrature error is bounded in $L_{h}^{2}$ by $C h|\delta n|_{L_{h}^{2}} \leq C|\delta x|_{L_{h}^{2}}$. We have now replaced the sum (7.9) with the corresponding sum without tildes, and obtained the two principal terms in (6.27).

Next we consider the difference which results from replacing $\tilde{x}_{j}, \tilde{x}_{\ell}$ with $x_{j}, x_{\ell}$ in the sum

$$
\sum \nabla G_{h}^{\pi}\left(\tilde{x}_{j}-\tilde{x}_{\ell}\right) \cdot N_{\ell}\left[\left(n_{\ell} \cdot n_{j}\right) w_{\ell}-w_{j}\right] h^{2} .
$$

The difference is a similar sum with $\delta \nabla G_{h}^{\pi}$ instead of $\nabla G_{h}^{\pi}$. We write $\delta \nabla G_{h}^{\pi}=$ $R_{2}\left(\alpha_{j}, \alpha_{\ell}\right) \cdot\left(\delta x_{j}-\delta x_{\ell}\right)$, where $R_{2}$ denotes an average of second derivatives of $G_{h}^{\pi}$ along the line from $x_{j}-x_{\ell}$ to $\tilde{x}_{j}-\tilde{x}_{\ell}$. Since the bracket term vanishes on the diagonal, Theorem 5.1 tells us that the $\delta x_{\ell}$ part of the error is bounded in $L_{h}^{2}$ by $C|\delta x|_{L_{h}^{2}}$. For the second part, we factor $\delta x_{j}$ out of the sum; the remaining sum is bounded, uniformly in $j$ and $h$, according to Lemma 5.4. Thus the second part of the error has the same bound as the first.

We are now left with the difference

$$
\sum \delta \nabla G_{h}^{\pi} \cdot \delta\left[N_{\ell}\left(\left(n_{\ell} \cdot n_{j}\right) w_{\ell}-w_{j}\right)\right] h^{2} .
$$

By considering cases we can see that this is of type $B(\delta x)$. From $\delta \nabla G_{h}^{\pi}$ we have $R_{2}$ times a factor of $\delta x_{j}$ or $\delta x_{\ell}$, and each term in the second factor has at least one difference in either $j$ or $\ell$. We discuss two specific terms. For the term

$$
\sum R_{2} \cdot \tilde{N}_{\ell}\left(\delta x_{j}\right)\left(\delta w_{j}\right) h^{2}=\left(\delta x_{j}\right)\left(\delta w_{j}\right) \sum R_{2} \cdot \tilde{N}_{\ell} h^{2}
$$

we note that $\left|\tilde{N}_{\ell}\right|$ is uniformly bounded, and $\delta w_{j}$ is uniformly $O(h)$. The last sum is $O\left(h^{-1}\right)$, uniformly in $j$; this can be seen from the pointwise estimates (2.4) for 
$D^{m} G_{h}$. Thus the term has the form $\delta x_{j}$ times a uniformly bounded function of $j$. Next we consider the term

$$
\sum R_{2} \cdot \tilde{N}_{\ell}\left(\delta x_{j}\right)\left(\delta n_{\ell}\right) \tilde{w}_{\ell} h^{2}
$$

We can estimate $|(\delta n) \tilde{w}|_{L_{h}^{2}}$ by $C h^{2}$. Then from Theorem 5.1, $h R_{2}$ is a bounded operator on $L_{h}^{2}$, so that the sum omitting the $\delta x_{j}$ factor is $O(h)$ in $L_{h}^{2}$, and therefore $O(1)$ in $L_{h}^{\infty}$. This is then multiplied by $\delta x_{j}$, and the resulting term is $B(\delta x)$. The other terms can be treated by the same methods.

Proof of (6.28). We have to find the difference between the sum

$$
-\sum D_{h, k} G_{h}^{\pi}\left(\tilde{x}_{j}-\tilde{x}_{\ell}\right) \tilde{X}_{k \ell}^{*} \cdot \tilde{n}_{j} \tilde{w}_{\ell}\left|\tilde{N}_{\ell}\right| h^{2}
$$

and the corresponding sum without tildes. We have written out $\nabla_{h}^{T} G_{h}^{\pi}$, recalling (1.23), with a sum over $k=1,2$ implicit. We begin with the sum

$$
-\sum D_{h, k} G_{h}^{\pi}\left(x_{j}-x_{\ell}\right) \tilde{X}_{k \ell}^{*} \cdot\left(\tilde{n}_{j}-\tilde{n}_{\ell}\right) \tilde{w}_{\ell}\left|\tilde{N}_{\ell}\right| h^{2}
$$

and add differences successively to remove the tildes. We have inserted $-\tilde{n}_{\ell}$; this does not affect the sum, since $\tilde{n}_{\ell} \perp \tilde{X}_{k \ell}^{*}$. We first replace $\tilde{n}_{j}$ with $n_{j}$, resulting in the difference, after a summation by parts,

$$
-\left(\delta n_{j}\right) \cdot \sum G_{h}^{\pi}\left(x_{j}-x_{\ell}\right) D_{h, k}^{*}\left[X_{k \ell}^{*} w_{\ell}\left|N_{\ell}\right|\right] h^{2}
$$

plus a remainder. The remainder has $\delta\left(X_{k \ell}^{*} w_{\ell}\left|N_{\ell}\right|\right)$, which is $O\left(h^{2}\right)$ in $L_{h}^{2}$, acted on by $D_{h, k} G_{h}^{\pi}$. Since $G_{h}^{\pi}$ is an operator of order -1 on $L_{h}^{2}$, by Theorem $5.1 D_{h, k} G_{h}^{\pi}$ is bounded, so that the image is $O\left(h^{2}\right)$ in $L_{h}^{2}$ and thus $O(h)$ in $L_{h}^{\infty}$. This is multiplied by $\delta n_{j}$, which is bounded in $L_{h}^{2}$ by $h^{-1}|\delta x|_{L_{h}^{2}}$, so that remainder term is of type $B(\delta x)$. As for the main term (7.22), the sum on the right is close to the integral in (6.28), after replacing $w_{\ell}$ by $\phi_{n}\left(\alpha_{\ell}\right)$ etc., with $O(h)$ quadrature error. This last error gives a term bounded by $h|\delta n|_{L_{h}^{2}}$ or $|\delta x|_{L_{h}^{2}}$.

Next we replace $\tilde{n}_{\ell}$ by $n_{\ell}$. The difference is

$$
\sum D_{h, k} G_{h}^{\pi}\left(x_{j}-x_{\ell}\right) X_{k \ell}^{*} \cdot\left(\delta n_{\ell}\right) w_{\ell}\left|N_{\ell}\right| h^{2}
$$

plus a remainder again with $\delta\left(X_{k \ell}^{*} w_{\ell}\left|N_{\ell}\right|\right)$. As before, the remainder is $B(\delta x)$. We can substitute in (7.23) for $\delta n$ from (6.12) and obtain the second term on the right in 6.28), up to a remainder which is $B(\delta x)$.

We now have reduced (7.20) to

$$
-\sum D_{h, k} G_{h}^{\pi}\left(x_{j}-x_{\ell}\right) \tilde{X}_{k \ell}^{*} \cdot\left(n_{j}-n_{\ell}\right) \tilde{w}_{\ell}\left|\tilde{N}_{\ell}\right| h^{2} .
$$

Now since $n_{j}-n_{\ell}$ vanishes at $j=\ell$, Theorem 5.2 tells us that the operator with kernel $\left(n_{j}-n_{\ell}\right) D_{h, k} G_{h}^{\pi}$ gains a derivative. Thus, to replace $\tilde{X}_{k \ell}^{*}\left|\tilde{N}_{\ell}\right|$, we estimate $\tilde{w} \delta\left(X_{k}^{*}|N|\right)$ in $H_{h}^{-1}$ by $|\delta x|_{L_{h}^{2}}$. The corresponding sum is then bounded by $|\delta x|_{L_{h}^{2}}$. To finish with (7.20), we replace $\tilde{w}_{\ell}$ with $w_{\ell}$, obtaining as the difference the first term on the right of (6.28).

Next we consider the difference

$$
\begin{aligned}
\sum \delta\left[D_{h, k} G_{h}^{\pi}\left(x_{j}-x_{\ell}\right)\right] X_{k \ell}^{*} \cdot n_{j} w_{\ell}\left|N_{\ell}\right| h^{2} & \\
= & \sum\left[\delta G_{h}^{\pi}\left(x_{j}-x_{\ell}\right)\right] \cdot n_{j} D_{h, k}^{*}\left[X_{k \ell}^{*} w_{\ell}\left|N_{\ell}\right|\right] h^{2} .
\end{aligned}
$$

We write $\delta G_{h}^{\pi}$ as $R_{1}\left(\alpha_{j}, \alpha_{\ell}\right) \cdot\left(\delta x_{j}-\delta x_{\ell}\right)$, where $R_{1}$ is an average of $D G_{h}^{\pi}$ from $x_{j}-x_{\ell}$ to $\tilde{x}_{j}-\tilde{x}_{\ell}$. For the $\delta x_{\ell}$ part, we conclude from the boundedness of $D G_{h}^{\pi}$ on 
$L_{h}^{2}$ that the sum is bounded by $|\delta x|_{L_{h}^{2}}$. For the second part, we bring $\delta x_{j}$ outside the sum. The remaining sum is uniformly bounded according to Lemma [5.4] and this term is $B(\delta x)$. We are left with more nonlinear terms, which can be handled as at the end of (6.27), after summing by parts to remove $D_{h, k}$ from $G_{h}^{\pi}$ as above.

Proof of (6.29). The difference has the form

$$
\delta(n \cdot \Sigma)=(\delta n) \cdot \Sigma+n \cdot \delta \Sigma+(\delta n) \cdot(\delta \Sigma)
$$

Since $\delta n_{j}$ is uniformly $O(h)$, by (6.6), it will be evident that the last term can be neglected after we have treated the second term. The sums in the first term are within $O(h)$ of the corresponding integrals, according to Theorem 3.7 (We use summation by parts.) The resulting error term is $B(h \delta n)=B(\delta x)$. The integrals can be converted to the integral in (6.29) by reversing the steps which led to (1.7). Now our task is to evaluate the second term in (7.26).

We treat the first sum in (6.29). As before, we begin with

$$
n_{j} \cdot \sum\left[D_{h} G_{h}^{\pi}, \tilde{X}\right]_{\ell} \times\left(\tilde{\nabla}_{h}^{T} \tilde{\phi}_{\ell}-\tilde{\nabla}_{h}^{T} \tilde{\phi}_{j}\right) h^{2},
$$

where $G_{h}^{\pi}=G_{h}^{\pi}\left(x_{j}-x_{\ell}\right)$ and $\tilde{\nabla}_{h}^{T} f=\sum_{k=1,2}\left(D_{h, k} f\right) \tilde{X}_{k}^{*}$. We remove the tildes in steps; we first replace $\tilde{\nabla}_{h}^{T} \tilde{\phi}_{\ell}$ with $\nabla_{h}^{T} \phi_{\ell}$. The difference is

$$
n_{j} \cdot \sum\left[D_{h} G_{h}^{\pi}, X\right]_{\ell} \times \delta\left(\nabla_{h}^{T} \phi_{\ell}\right) h^{2}
$$

plus a remainder with products of $\delta$ 's. This remainder is $B(\delta x)$; to see this, we note that $D_{h} G_{h}^{\pi}$ is a bounded operator on $L_{h}^{2}$ and products such as $\left(\delta X_{1 \ell}\right)\left(D_{h, 2} \delta \phi_{\ell}\right)$ are bounded in $L_{h}^{2}$ by $|\delta x|_{L_{h}^{2}}$, using (6.6). In (17.28) we can substitute for $\delta\left(\nabla_{h}^{T} \phi_{\ell}\right)$ from (6.14), obtaining, up to a negligible remainder,

$$
\begin{aligned}
\sum n_{j} \cdot\left[D_{h} G_{h}^{\pi}, X\right]_{\ell} & \times \nabla_{h}^{T}\left(\delta \phi_{\ell}-\nabla_{h}^{T} \phi_{\ell} \cdot \delta x_{\ell}\right) h^{2} \\
& +\sum\left(\left[D_{h} G_{h}^{\pi}, X\right]_{\ell} \times n_{\ell}\right) \cdot n_{j}\left(\nabla_{h}^{T}\left(n_{\ell} \cdot \delta x_{\ell}\right) \cdot \nabla_{h}^{T} \phi_{\ell}\right) h^{2} .
\end{aligned}
$$

In the first sum we substitute $n_{j}=n_{\ell}+\left(n_{j}-n_{\ell}\right)$ and split the sum into two parts. The $n_{\ell}$-part can be converted to the first term on the right in (6.29) using a vector identity as in (1.6). For the remaining part in the first sum of (7.29), we have a smooth factor which vanishes at $\ell=j$, and we can use Theorem 5.2 to show that the sum is $B(\delta x)+B(\delta \phi)$. As for the second sum in (7.29), we note that the cross product is tangential at $x_{\ell}$, so that the scalar product with $n_{j}$ is zero when $\ell=j$. Thus we can use Theorem 5.2 again to bound this term by $|\delta x|_{L_{h}^{2}}$.

Next we replace $\tilde{\nabla}_{h}^{T} \tilde{\phi}_{j}$ in (7.27) by $\nabla_{h}^{T} \phi_{j}$. The difference is

$$
n_{j} \cdot \delta\left(\nabla_{h}^{T} \phi_{j}\right) \times \sum\left[D_{h} G_{h}^{\pi}, X\right]_{\ell} h^{2},
$$

except for an error nonlinear in the $\delta$ 's which is again negligible. The sum in (7.30) is within $O(h)$ of an integral which is zero; see (1.5), (1.8), (1.28). The term with the quadrature error is of order $h\left|\delta \nabla_{h}^{T} \phi\right|_{L_{h}^{2}}$, which in view of (6.14) is $B(\delta \phi)+B(\delta x)$. We have now reduced (7.27) to

$$
n_{j} \cdot \sum\left[D_{h} G_{h}^{\pi}, \tilde{X}\right]_{\ell} \times\left(\nabla_{h}^{T} \phi_{\ell}-\nabla_{h}^{T} \phi_{j}\right) h^{2} .
$$

The last factor vanishes when $\ell=j$, and we can therefore use Theorem 5.2 to replace $\tilde{X}_{\ell}$ with $X_{\ell}$, with a difference $B(\delta x)$, thus completing the treatment of (7.27). 
The most important remaining term from the first sum on the left in (6.29) is

$$
n_{j} \cdot \sum\left[\delta\left(D_{h} G_{h}^{\pi}\right), X\right]_{\ell} \times\left(\nabla_{h}^{T} \phi_{\ell}-\nabla_{h}^{T} \phi_{j}\right) h^{2} .
$$

This can be treated similarly to the corresponding term in 6.28). The other terms are nonlinear and can be handled like earlier cases. The variation of the second sum can be treated by techniques as for 6.27), but more simply. We need to use the fact that $\nabla_{h}^{T} \phi_{j}$ is multiplied by a discretization of an integral which is zero by (1.5).

Proof of Lemma 6.7. The operator $\tilde{\mathcal{K}}_{h}$ differs from $\mathcal{K}_{h}$ by an operator which is $O(h)$. This can be shown by estimates related to (6.27), 6.28) but simpler. By the remarks in the proof of Lemma $6.1,2 \tilde{\mathcal{K}}_{h}$ then has spectral radius less than one, and it follows that the discrete integral equation can be solved by simple iteration.

\section{REFERENCES}

1. C. Anderson and C. Greengard, On vortex methods, SIAM J. Numer. Anal. 22 (1985), 413440. MR 86j:76016

2. V. K. Andreev, Stability of unsteady motions of a fluid with a free boundary, VO Nauka, Novosibirsk, 1992 (in Russian). MR 94j:76001

3. G. Baker, D. Meiron, and S. Orszag, Generalized vortex methods for free-surface flow problems, J. Fluid Mech. 123 (1982), 477-501. MR 84a:76002

4. G. Baker, D. Meiron, and S. Orszag, Boundary integral methods for axisymmetric and threedimensional Rayleigh-Taylor instability problems, Physica D 12 (1984), 19-31. MR 87h:76071

5. J. T. Beale, T. Y. Hou and J. S. Lowengrub, Growth rates for the linearized motion of fluid interfaces away from equilibrium, Comm. Pure Appl. Math. 46 (1993), 1269-1301. MR 95c:76016

6. J. T. Beale, T. Y. Hou and J. S. Lowengrub, Convergence of a boundary integral method for water waves, SIAM J. Numer. Anal. 33 (1996), 1797-1843. MR 98b:76009

7. J. T. Beale and A. Majda Vortex methods, I: Convergence in three dimensions, Math. Comp. 39 (1982), 1-27. MR 83i:65069a

8. J. T. Beale and A. Majda, High order accurate vortex methods with explicit velocity kernels, J. Comput. Phys. 58 (1985), 188-208.

9. J. Broeze, E. F. G. Van Daalen, and P. J. Zandbergen, A three-dimensional panel method for nonlinear free surface waves on vector computers, Comput. Mech. 13 (1993), 12-28.

10. D. Colton and R. Kress, Integral Equation Methods in Scattering Theory, Wiley, New York, 1983. MR 85d:35001

11. G. H. Cottet and P. A. Raviart, Particle methods for the one-dimensional Vlasov-Poisson equations, SIAM J. Numer. Anal. 21 (1984), 52-76. MR 85c:82048

12. J. Goodman, T. Y. Hou and J. Lowengrub, Convergence of the point vortex method for the 2-D Euler equations, Comm. Pure Appl. Math. 43 (1990), 415-430. MR 91d:65152

13. I. S. Gradshteyn and I. M. Ryzhik, Table of Integrals, Series, and Products, Academic Press, New York, 1980. MR 81g:33001

14. L. Greengard and V. Rokhlin, A new version of the fast multipole method for the Laplace equation in three dimensions, Acta Numer. 6 (1997), 229-269. MR 99c:65012

15. B. Gustafsson, H. O, Kreiss, and J. Oliger, Time dependent problems and difference methods, Wiley, New York, 1995. MR 97c:65145

16. O. Hald, The convergence of vortex methods, II, SIAM J. Numer. Anal. 16 (1979), 726-755. MR 81b:76015b

17. D. Haroldsen and D. Meiron, Numerical calculation of three dimensional interfacial potential flows using the point vortex method, SIAM J. Sci. Comput. 20 (1999), 648-683. MR 99c:76010

18. T. Y. Hou, Z. Teng, and P. Zhang, Well-posedness of linearized motion for 3-D water waves far from equilibrium, Commun. P.D.E. 21 (1996), 1551-1585. MR 98c:76013

19. T. Y. Hou and P. Zhang, Stability of a boundary integral method for 3-D water waves, submitted to SIAM J. Numer. Anal.

20. R. E. Kleinman and G. F. Roach, Boundary integral equations for the three-dimensional Helmholtz equation, SIAM Review 16 (1974), 214-236. MR 52:988 
21. M. S. Longuet-Higgins and E. D. Cokelet, The deformation of steep surface waves on water, I. A numerical method of computation, Proc. Roy. Soc. London A 350 (1976), 1-26. MR 53:15091

22. J. Lowengrub, M. Shelley, and B. Merriman, High-order and efficient methods for the vorticity formulation of the Euler equations, SIAM J. Sci. Comput. 14 (1993), 1107-1142. MR 94e:76065

23. J. N. Lyness, An error functional expansion for $N$-dimensional quadrature with an integrand function singular at a point, Math. Comp. 30 (1976), 1-23. MR 53:11976

24. J. N. Lyness, A survey of numerical cubature over triangles, in Proc. Symp. Appl. Math. 48, A.M.S., Providence, 1994. MR 95j:65021

25. A. Majda, J. McDonough and S. Osher, The Fourier method for nonsmooth initial data, Math. Comp. 32 (1978), 1041-1081. MR 80a:65197

26. R. D. Richtmyer and K. W. Morton, Difference Methods for Initial Value Problems, 2nd ed., Wiley, New York, 1967. MR 36:3515

27. J. E. Romate, The numerical simulation of nonlinear gravity waves, Engrng. Analysis Bdry. Elts., 7 (1990), 156-166.

28. J. E. Romate and P. J. Zandbergen Boundary integral equation formulations for free-surface flow problems in two and three dimensions, Comput. Mech. 4 (1989), 267-282.

29. J. Strain, Fast potential theory. II. Layer potentials and discrete sums, J. Comput Phys. 99 (1992), 251-270. MR 93b:65197

30. M. Taylor, Pseudodifferential Operators, Princeton Univ. Press, Princeton, NJ, 1981. MR 82i:35172

31. M. Taylor, Partial Differential Equations, Springer, New York, 1996. MR 98b:35002

32. W. Tsai and D. Yue, Computations of nonlinear free-surface flows, Ann. Rev. Fluid Mech. 28 (1996), 249-278. MR 96i:76086

33. T. Vinje and P. Brevig, Numerical simulation of breaking waves, Adv. Water Resources 4 (1981), 77-82.

34. Sijue $\mathrm{Wu}$, Well-posedness in Sobolev spaces of the full water wave problem in 2-D, Invent. Math. 130 (1997), 39-72. MR 98m:35167

35. Sijue $\mathrm{Wu}$, Well-posedness in Sobolev spaces of the full water wave problem in 3-D, J. Amer. Math. Soc. 12 (1999), 445-495. CMP 99:08

Department of Mathematics, Duke University, Durham, NC 27708-0320

E-mail address: beale@math.duke.edu 